\title{
Radar-based Monitoring of Vital Signs: A Tutorial Overview
}

This paper was downloaded from TechRxiv (https://www.techrxiv.org).

\section{LICENSE}

$\mathrm{CCO}$

SUBMISSION DATE / POSTED DATE

22-02-2022 / 01-03-2022

\section{CITATION}

Paterniani, Giacomo; Sgreccia, Daria; DAVOLI, ALESSANDRO; Guerzoni, Giorgio; Di Viesti, Pasquale; Valenti, Anna Chiara; et al. (2022): Radar-based Monitoring of Vital Signs: A Tutorial Overview. TechRxiv. Preprint. https://doi.org/10.36227/techrxiv.19212918.v1

$\mathrm{DOI}$ 


\title{
Radar-based Monitoring of Vital Signs: A Tutorial Overview
}

\author{
Giacomo Paterniani ${ }^{1}$, Daria Sgreccia ${ }^{2}$, Alessandro Davoli $^{1}$, Giorgio Guerzoni $^{1}$, \\ Pasquale Di Viesti ${ }^{1}$, Anna Chiara Valenti ${ }^{2}$, Marco Vitolo ${ }^{2}$, Giorgio M. Vitetta ${ }^{1}$, and \\ Giuseppe Boriani ${ }^{2}$
}

\author{
${ }^{1}$ University of Modena and Reggio Emilia, Department of Engineering "E. Ferrari", Via P. Vivarelli 10/1, 41125 \\ Modena (Italy) and Consorzio Nazionale Interuniversitario per le Telecomunicazioni (CNIT). \\ ${ }^{2}$ University of Modena and Reggio Emilia, Cardiology Division, Department of Biomedical, Metabolic and Neural \\ Sciences, via Giuseppe Campi, 287, 41125 Modena (Italy).
}

\begin{abstract}
In the last years, substantial attention has been paid to the use of radar systems in health monitoring, thanks to the availability of both low cost radar devices and computationally efficient algorithms for processing their measurements. In this manuscript, a tutorial overview of radar-based monitoring of vital signs is provided. More specifically, we first focus on the available radar technologies and on the signal processing algorithms developed for the estimation of vital signs. Then, we illustrate various specific applications of radar systems to health monitoring and some relevant research trends in this field. Finally, we provide some useful guidelines that should be followed in the selection of radar devices for vital sign monitoring and in their use.
\end{abstract}

Index terms - Radar, signal processing, MIMO, health monitoring, vital signs.

\section{Introduction}

Monitoring human vital signs, like heart and respiration rates, represents a routine practice to detect patient deterioration. Changes in vital signs can reveal the existence of serious medical problems; for this reason, early identification of these changes can improve survival rates in several conditions [1]. Vital sign monitoring is often accomplished by means of wearable health devices [2]; this is due to the fact that these devices enable continuous monitoring during daily activities. However, in various situations, like in the case of infected patients, or of patients suffering from mental illness or affected by severe burns or injuries, the use of wearable sensors is not possible or recommended. In such cases, the use of non-contact monitoring devices, like radar systems, can help healthcare professionals by providing critical information about patient state [3]. The application of radar devices to this field and, in particular, to the estimation of the heart and respiration rates, has become an active research area in recent years $[4,5]$. Actually, the first experimental results in this field date back to 1975, when the use of short-range radar technology was proposed to non-invasively acquire respiratory information by comparing a microwave signal with its echo reflected from the chest of a patient [6]. In the following years, the possibility of employing radar systems for the wireless detection of the physiological movements due to both heartbeat and respiration has been shown [7-10]. This has motivated the investigation of the use of this technology in a number of medical applications, including adult and neonatal sleep monitoring [11], [12], disaster medicine (e.g., in the detection of human vital signs under rubbles after earthquakes [13]) and lung cancer radiotherapy [14].

In the last two decades, few review articles about radar-based monitoring of vital signs have been published [10,15-18]; however, they have a limited scope since they concern the use of specific technologies. In fact, on the one hand, $[10,15,16]$ and [17] focus on continuous wave $(\mathrm{CW})$ Doppler radars, ultra-wide band (UWB) and radars equipped with a single transmit/receive antenna (i.e., single-input single-output, SISO, radars), respectively. On the other hand, [18] takes into consideration radars equipped with antenna arrays (i.e., multiple-input multiple-output, MIMO, radars) and illustrates the advantages they offer with respect to 
their SISO counterparts. This has motivated the writing of this manuscript, that aims at offering a tutorial overview of radar-based monitoring of vital signs and at providing some essential tips for its use in a research laboratory. More specifically, in the remaining part of the manuscript, we first provide essential information about radar-based monitoring, cardiovascular and respiration physiology, and the modelling of chest displacement. Then, we focus on the available radar technologies, and describe various radar architectures and signal processing methods developed for radar-based estimation of vital signs; in our description, all the available options are taken into consideration, and their pros and cons are illustrated. The study of these topics allows us to lay the foundations for understanding specific applications of radar-based monitoring and relevant research trends in this field. Our discussion on this issues is followed by various considerations formulated in the light of the experience acquired in our experimental activities on radar-based monitoring; such considerations concern the essential technical requirements that radar devices employed in this field should have and some essential guidelines to be followed in conducting experimental campaigns.

This manuscript is organized as follows. In Section II. the basic principles, challenges and objectives of radar-based monitoring of vital signs are illustrated. In Section III. the physiological fundamentals of human cardiovascular and respiration activities are provided and simple mathematical models describing the dynamics of chest displacement due to these activities are described. Section IV. is devoted to the four radar technologies employed in radar-based monitoring of vital signs, and to SISO and MIMO radar architectures; for each architecture, simple mathematical models are provided for the samples of the baseband signal received in the presence of a single point target. An overview of the most important deterministic and learning-based signal processing techniques employed for vital signs monitoring is offered in Section V.. The application of these techniques to heart and breath rate estimation, heart sound monitoring and heart rate variability estimation is discussed in Section VI.. Current research trends on radar-based monitoring of vital signs are illustrated in Section VII., whereas some basic guidelines to be followed in conducting experimental activities in this field are given in Section VIII.. Finally, some conclusions are offered in Section IX..

A list of the acronyms employed throughout the manuscript is provided in Table 1.

\section{Radars for Vital Sign Monitoring: Basic Principles, Objectives and Challenges}

A system for radio detection and ranging (i.e., briefly, a radar system) is an electronic system designed to estimate the spatial coordinates (i.e., the distance and the angular coordinates) and/or the velocity of objects (called targets), that, thanks to their electrical conductivity, are able to reflect back the electromagnetic waves it generates. Any radar system consists of a transmitter and a receiver which, in the applications considered in this manuscript, are integrated in the same electronic device. The transmitter generates radio waves with known properties and radiates them along a predetermined direction using a single transmit antenna or multiple transmit antennas (i.e., an antenna array), whereas the receiver captures the waves reflected back by the above mentioned targets.

The measurement of the distance (i.e., of the range) of any target from a given radar system is based on the estimation of the propagation delay of the received waves, whereas that of its velocity on some structural changes in such waves; for instance, if a target is approaching the radar or is moving away from it, a variation in the frequency of the received radio waves is observed because of the Doppler effect. Target range and velocity can be estimated by radar systems equipped with a single transmit (TX) and single receive (RX) antenna. The measurement of the angular coordinates of any target requires, instead, the availability of at least two RX antennas, that is of an antenna array at the receive side, since it is equivalent to the estimation of the direction of arrival (DOA) of the electromagnetic waves impinging on the radar receiver; note that, in general, the use of a larger number of antenna elements forming the RX array results in a better angular resolution, i.e. in more accurate estimates of the angular coordinates of the surrounding targets (and, consequently, in more detailed radar images). Modern radars, and especially those employed for vital signs monitoring, are quite small and compact in size, since they operate at very high frequencies and, in particular, in the microwave spectrum ${ }^{1}$. The basic components of these devices are a digital control board and a radio frequency (RF) front-end, whose implementation is usually based on a monolithic microwave

\footnotetext{
${ }^{1}$ This portion of electromagnetic spectrum is often defined as interval of frequencies ranging from $1 \mathrm{GHz}$ to $100 \mathrm{GHz}$ (corresponding to wavelengths between $0.3 \mathrm{~m}$ and $3 \mathrm{~mm}$ ).
} 
Table 1: Table of the acronyms defined in the manuscript.

\begin{tabular}{|c|c|}
\hline $\mathrm{ADC}$ & Analog to Digital Converter \\
\hline $\mathrm{AD}$ & Arctangent Demodulation \\
\hline AI & Artificial Intelligence \\
\hline ANC & Adaptive Noise Cancellation \\
\hline API & Application Programming Interface \\
\hline ASIC & Applied Specific Integrated Circuit \\
\hline AWGN & Additive White Gaussian Noise \\
\hline BR & Breath rate \\
\hline BPF & Band Pass Filtering \\
\hline CFAR & Constant False Alarm Rate \\
\hline CV & Correlation of Variation \\
\hline CNN & Convolutional Neural Network \\
\hline CSD & Complex Signal Demodulation \\
\hline CPU & Central Processing Unit \\
\hline CW & Continuous Wave \\
\hline CWT & Continuous Wavelet Transform \\
\hline DACM & Differentiate And Cross Multiplier \\
\hline DC & Direct Current \\
\hline DFT & Discrete Fourier Transform \\
\hline DSP & Digital Signal Processor \\
\hline DL & Deep Learning \\
\hline DOA & Direction of Arrival \\
\hline DT & Decision Tree \\
\hline ECG & Electrocardiogram \\
\hline EMD & Empirical Mode Decomposition \\
\hline FMCW & Frequency Modulated Continuous Wave \\
\hline FOV & Field of View \\
\hline FPGA & Field Programmable Gate Array \\
\hline FFT & Fast Fourier Transform \\
\hline GPU & Graphic Processing Unit \\
\hline HR & Heart Rate \\
\hline HRV & Heart Rate Variability \\
\hline IDFT & Inverse DFT \\
\hline IFFT & Inverse $\mathrm{FFT}$ \\
\hline IMF & Intrinsic Mode Function \\
\hline IR-UWB & Impulse Radio Ultra-Wideband \\
\hline ISM & Industrial Scientific and Medical \\
\hline IWR & Industrial mmWave Radar \\
\hline $\mathrm{K}-\mathrm{NN}$ & K - Nearest Neighbour \\
\hline LB & Leaning Based \\
\hline
\end{tabular}

\begin{tabular}{|c|c|}
\hline LDA & Linear Discriminant Analysis \\
\hline LNA & Low Noise Amplifier \\
\hline LO & Local Oscillator \\
\hline LS & Least Square \\
\hline LSTM & Long Short Term Memory \\
\hline MAE & Mean Absolute Error \\
\hline MEMS & Micro Electro-Mechanical System \\
\hline MF & Matched Filter \\
\hline MIMO & Multiple Input Multiple Output \\
\hline ML & Machine Learning \\
\hline MMIC & Monolithic Microwave Integrated Circuit \\
\hline NN & Normal to Normal \\
\hline P2G & Position To Go \\
\hline PA & Power Amplifier \\
\hline PAE & Peak Absolute Error \\
\hline PRI & Pulse Repetition Interval \\
\hline RADAR & Radio Detection And Ranging \\
\hline RBM & Random Body Movement \\
\hline $\mathbf{R F}$ & Radio Frequency \\
\hline RHS & Right Hand Side \\
\hline RMSE & Root Mean Square Error \\
\hline RMSSD & Root Mean Square Successive Difference \\
\hline RX & Receive \\
\hline SDNN & Standard Deviation of Normal to Normal \\
\hline SFCW & Stepped Frequency Continuous Wave \\
\hline SISO & Single Input Single Output \\
\hline SNR & Signal-to-Noise Ratio \\
\hline SVM & Support Vector Machine \\
\hline TDM & Time Division Multiplexing \\
\hline TI & Texas Instrument \\
\hline $\mathbf{T X}$ & Transmit \\
\hline ULA & Uniform Linear Array \\
\hline URA & Uniform Rectangular Array \\
\hline UWB & Ultra-wideband \\
\hline VA & Virtual Antenna \\
\hline VGA & Voltage Gain Amplifier \\
\hline VCO & Voltage Controlled Oscillator \\
\hline VHSIC & Very High Speed Integrated Circuit \\
\hline TRI & Triangular Index \\
\hline
\end{tabular}


integrated circuit (MMIC) and small-sized patch antennas [19]. If microwave frequencies are used, small size TX and RX antennas are implemented and electromagnetic signals characterized by a very large bandwidth and small wavelength are radiated; the last features make it possible to achieve an excellent range solution and detect small movements.

Radar-based monitoring of vital signs is based on the idea that the chest-wall of human bodies reflects the electromagnetic waves generated by a radar placed in front of it and that its quasi periodic vibrations, resulting from respiration and heartbeat, modulate such waves. Therefore, in principle, essential information about vital signs, i.e. heart and breath rates, can be extracted from the reflected electromagnetic waves [20-22] and a fundamental objective, namely contactless monitoring of vital signs, can be achieved. Note also that, compared with traditional methods, such as pneumotachography and electrocardiography, radar systems make continuous and timely breath rate (BR) and heart rate (HR) monitoring possible without entailing an additional work load for nurses. For these reasons, radars represent a favourable option for hospital monitoring, especially in the case of severe burn or infectious disease patients, sudden infant death syndrome monitoring, sleep apnoea monitoring, elderly home healthcare and psychology studies. Moreover, radar signals can be processed to extract more refined medical information and, in particular, to detect anomalous alterations in the sequence of heart beats.

Even if the usefulness of radar systems in vital sign monitoring is now globally recognized and a wide literature about such systems is available, various challenges concerning signal processing techniques for vital signs extraction are still open in this research field; here, we limit to mention:

a) The estimation of heart rate - As illustrated in Paragraph III.B., the vibrations due to heartbeat are significantly weaker than those originating from respiration. For this reason, the contribution of the first phenomenon to the radar signal may be hidden by that related to the second (and much stronger) one. This makes the task of estimating HR much harder than that estimating BR; additional details about this issue can be found in Paragraphs VI.A. and VIII.C..

b) The identification of anomalous alterations of heart beats - This challenge concerns the possibility of estimating HR variability (HRV) and detecting heart sounds; these issues are discussed in Paragraphs VI.C. and VII.C., respectively.

c) The simultaneous observation of the vital signs of multiple people - In principle, MIMO radar systems can be exploited to detect and estimate the vital signs of multiple people located in a restricted area (e.g., in the same room; see Paragraph V.B. for further details). However, experimental results supporting the feasibility of this idea and involving a significant number of people are still missing in the technical literature (e.g., see [18, Table I, Sect. IV]).

Finally, it is worth mentioning that the experimental results available in the technical literature about radar-based monitoring of vital signs concern heterogeneous radar technologies and that there is not a broad consensus on the best technology to be adopted in real world systems. For this reason, it is important to analyse the prons and cons of each option available on the market; this issue is discussed in Section IV..

\section{Physiological Fundamentals and Mathematical Modelling}

In this section we first provide readers with the physiological fundamentals of heart and lung functions. Then, we concentrate on the movements of the chest surface in human beings and illustrate some mathematical models describing them.

\section{III.A. Basics of cardiovascular and respiration physiology}

The human heart is made of two separated systems, called left and right sides. Each side consists of two chambers, namely an atrium and a ventricle, which are separated and connected by an atrioventricular valve. The main function of the left side is to pump oxygenated blood through the aorta and the other arteries to peripheral tissues and organs. The right side, instead, is in charge of pumping deoxygenated blood through pulmonary arteries to lungs. Each side is connected to arteries through the so called semilunar valves.

The cardiac cycle consists of a rhythmic sequence of contractions (systoles) and relaxations (diastoles) of the heart; this events occur simultaneously in the left and right sides. During each cardiac cycle, sounds are generated by the action of the heart muscle and the vibrations of the cardiac valves. In the case of an healthy 


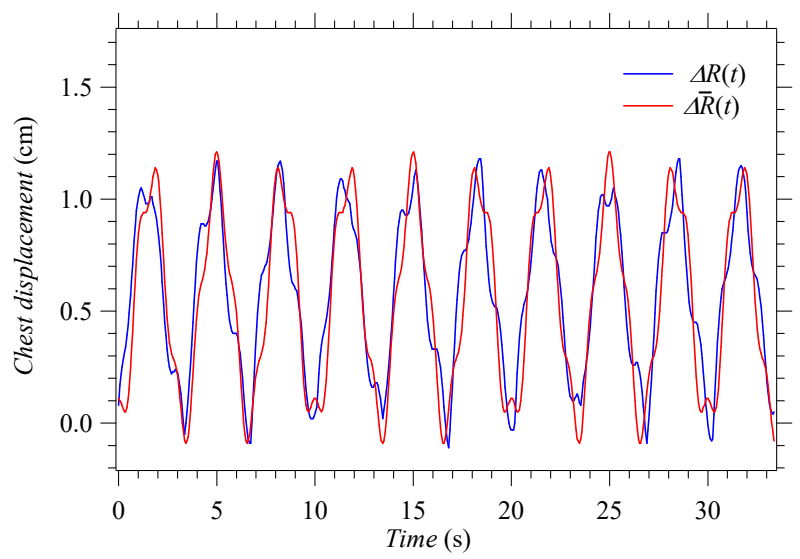

(a)

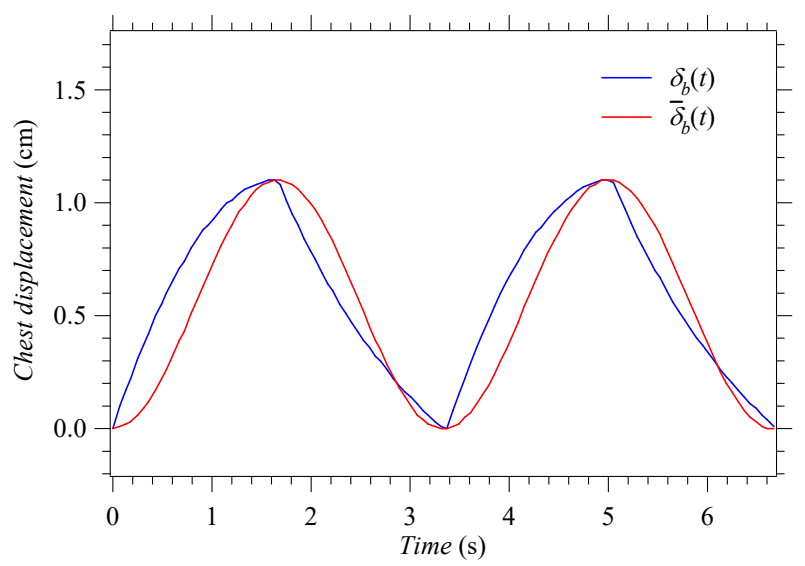

(c)

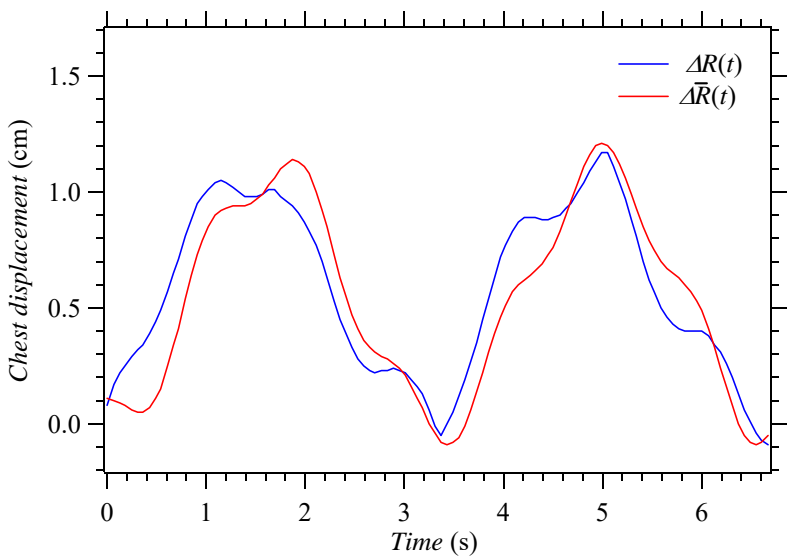

(b)

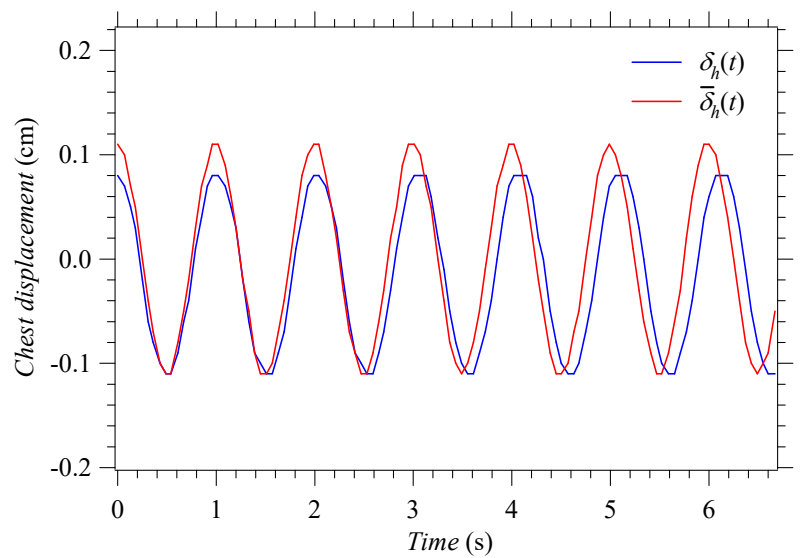

(d)

Figure 1: Representation of: a) a computer generated chest displacement (multiple periods of the breathing activity are considered); b) a computer generated chest displacement (a couple of consecutive periods of the breathing activity are considered); c) the contributions due to the breathing activity; d) the contributions due to the heart activity. In all these figures, a blue (red) line is used to identify the signals generated on the basis of $(1)((6))$.

adult heart, two heart sounds are detected. The first one is caused by the contraction of the ventricular muscle during systoles and the closing of atrioventricular valves. The second heart sound, instead, is due to the closure of aortic and pulmonary valves. Hemodynamic properties and HR can be determined by recording heart sounds. As a matter of fact, such sounds can help the physician in diagnosis of potential cardiovascular diseases [23,24]. The physiologic HR at rest is 60 beats per min (bpm) up to $100 \mathrm{bpm}$; values below this range (above it) characterise the so called bradycardia (tachycardia).

A breathing cycle consists of two consecutive phases, known as inspiration and expiration. In the first phase, thanks to the contraction of the diaphragm and of the intercostal muscles, the thoracic volume increases; this results in a sub-atmospheric pressure that allows air to flow through the airways into the lungs. Then, air oxygen is absorbed into the blood and carbon dioxide is transferred from the blood to the inhaled air through the alveolar-capillary membrane. Expiration, instead, is caused by the elastic recoil of the lungs and relaxing muscles. In this phase, the deoxygenated air can flow out of the lungs by increased pressure $[25,26]$. The physiologic $\mathrm{BR}$ at rest is 12 up to 25 acts per minute, whereas values below this range (above it) characterise the so called bradypnea (tachypnoea). 


\section{III.B. Modelling of chest displacement}

Let us focus now on the problem of modelling the chest displacement of an arbitrary patient. As illustrated in the previous paragraph, his/her inspiration (expiration) phase produces an expansion (compression) of the thoracic wall. Moreover, the vibrations due to his/her heart beat overlap with the thoracic breathing movement. In principle, the time evolution of both the thoracic breathing movement and the cardiac vibrations of the considered patient can be measured by a radar system placed in front of his/her chest at a fixed distance. In the absence of random and large-scale body movements, the displacement $\Delta R(t)$ of the chest surface measured by the radar system at the instant $t$ can be modelled as [27]

$$
\Delta R(t) \triangleq R(t)-R_{0}=\delta_{b}(t)+\delta_{h}(t),
$$

where $R(t)\left(R_{0}\right)$ is the radar-chest distance at time $t$ (distance in the absence of respiration) and $\delta_{b}(t)\left(\delta_{h}(t)\right)$ represents the breath (heart) contribution to $\Delta R(t)$. It is important to stress that:

a) the displacement $\delta_{b}(t)\left(\delta_{h}(t)\right)$ is usually assumed to be periodic with period $T_{\mathrm{BR}}\left(T_{\mathrm{HR}}\right)$, with $T_{\mathrm{BR}}>$ $T_{\mathrm{HR}}$;

b) the contribution of $\delta_{h}(t)$ to the displacement $\Delta R(t)$ is usually small with respect to that due to $\delta_{b}(t)$; in fact, if $\delta_{b, M}\left(\delta_{h, M}\right)$ denotes the maximum absolute value of $\delta_{b}(t)\left(\delta_{h}(t)\right)$, it is known that $2 \leq \delta_{b, M} \leq 5 \mathrm{~cm}$ and $5 \leq \delta_{h, M} \leq 15 \mathrm{~mm}[28,29]$.

An accurate model of the breath displacement $\delta_{b}(t)$ within each period has been proposed in [30]; according to this model, the displacement in the inspiration phase is described by the parabolic profile

$$
\delta_{b}(t)=-\frac{\delta_{b, M}}{T_{i} T_{e}}\left(t-t_{0}\right)^{2}+\frac{\delta_{b, M} T_{B R}}{T_{i} T_{e}}\left(t-t_{0}\right),
$$

with $t \in\left[t_{0}, t_{0}+T_{i}\right)$, whereas that in the expiration phase by the exponential profile

$$
\delta_{b}(t)=\frac{\delta_{b, M} \exp \left(-\frac{T_{e}}{\tau}\right)}{1-\exp \left(-T_{e} / \tau\right)}\left[\exp \left(-\frac{\left(t-t_{0}\right)-T_{B R}}{\tau}\right)-1\right]
$$

with $t \in\left[t_{0}+T_{i}, t_{0}+T_{\mathrm{BR}}\right)$; here, $t_{0}$ is the initial instant of the considered breathing period, $T_{i}\left(T_{e}\right)$ is the duration of the inspiration (expiration) phase, $\tau$ is the time constant of the expiratory profile; note that $T_{\mathrm{BR}}=T_{i}+T_{e}$. The displacement due to the cardiac activity, instead, can be modelled as [27]

$$
\delta_{h}(t)=\delta_{h, M} \cos \left(w_{1}\left(t-t_{1}\right)+\gamma_{h}(t)\right) \exp \left(-\frac{\left(\left(t-t_{1}\right)-a_{2}\right)^{2}}{a_{3}}\right),
$$

with

$$
\gamma_{h}(t)=a_{1} \sin \left(w_{2}\left(t-t_{1}\right)\right)
$$

and $t \in\left[t_{1}, t_{1}+T_{\mathrm{HR}}\right)$; here, $t_{1}$ is the initial instant of the considered heart beat, $\left\{a_{k} ; k=1,2,3\right\}$ and $\left\{w_{l}\right.$; $l=1$ and 2$\}$ are tunable and fixed parameters, respectively. Note that this model allows to account for heart rate variability (HRV) through the function $\gamma_{h}(t)$, i.e. for the changes in the time interval between consecutive beats ${ }^{2}$.

The chest displacement generated on the basis of (1)-(4) is exemplified by Fig. 1, where the contributions due to breathing and cardiac activities are also shown. In this case, the following values of the model parameters have been selected: $T_{\mathrm{BR}}=0.3 \mathrm{~s}, T_{\mathrm{HR}}=1 \mathrm{~s}, T_{i}=0.5 T_{\mathrm{BR}}, T_{e}=T_{i}, \delta_{b, M}=1.1 \mathrm{~cm}, \tau=T_{e}$, $a_{1}=\pi / 16, a_{2}=0.5, a_{3}=0.8, \delta_{h, M}=0.11 \mathrm{~cm}, w_{1}=2 \pi / T_{\mathrm{HR}} \mathrm{rad}, w_{1}=w_{2}=2 \pi / T_{\mathrm{BR}} \mathrm{rad} / \mathrm{s}$. Moreover, in $(2)-(5), t_{0}=\left(k_{b}-1\right) T_{\mathrm{BR}},\left(t_{1}=\left(k_{h}-1\right) T_{\mathrm{HR}}\right)$, with $k_{b}=0,1, \ldots, N_{b}-1\left(k_{h}=0,1, \ldots, N_{h}-1\right), N_{b}=\left\lfloor T_{F} / T_{\mathrm{BR}}\right\rfloor$ $\left(N_{h}=\left\lfloor T_{F} / T_{\mathrm{HR}}\right\rfloor\right)$ and $T_{F}$ is the duration of the whole observation interval.

Although the models illustrated above are accurate, a simpler representation of the heart and breathing profile has been adopted by various researchers $[21,31,32]$. More specifically, if $T_{i}$ is assumed to be equal $T_{e}$, the approximate model

$$
\Delta R(t) \approx \Delta \bar{R}(t)=\bar{\delta}_{b}(t)+\bar{\delta}_{h}(t),
$$

\footnotetext{
${ }^{2}$ Additional details about HRV are available in Par. VII.C..
} 
with

$$
\bar{\delta}_{b}(t) \triangleq \frac{\delta_{b, M}}{2}\left[1-\cos \left(w_{b} t\right)\right]
$$

and

$$
\bar{\delta}_{h}(t) \triangleq \delta_{h, M} \cos \left(w_{h} t\right),
$$

can be employed in place of that expressed by (1); here, $w_{b}=2 \pi / T_{\mathrm{BR}}\left(w_{h}=2 \pi / T_{\mathrm{HR}}\right)$ represents the $\mathrm{HR}$ (BR). An example of chest displacement generated according to (6) is provided in Fig. 1, where the contributions due to breathing and cardiac activities are also shown. From this figure it can be easily inferred that the results obtained on the basis of the models (1) and (6) are not so different; however, we should not forget that the model (6) is unable to account for the presence of both HRV and all the frequency components ${ }^{3}$ observed in the spectrum of the received signal.

Finally, it is worth mentioning that various sensors, such as pressure belts, fiber Bragg gratings and inertial sensors, can be exploited to monitor surface chest motion (some examples of commercial wearable sensors are described in Paragraph VIII.B.); however, all these sensors require to be worn by the patient under test. In a measurement campaign for radar-based monitoring, one of these sensors can be used as reference; this allows to separate the cardiac activity from the dominant breathing dynamics. In fact, as already mentioned above, the contribution of heart beats to surface chest motion is relatively small with respect to the that due to respiration. Moreover, the spectral components of heart motion may overlap with the respiratory harmonics; this makes separating the former contribution from the latter one really challenging.

\section{Radar Systems: Technologies and Architectures}

In this section, after providing a classification of the radar technologies employed for vital sign monitoring, some architectures of radar systems equipped with single and multiple TX/RX antennas are illustrated.

\section{IV.A. Radar technologies and classification}

Radar systems can be divided into two categories on the basis of the mechanism according to which the waveform they radiate is generated; more specifically, the first category if made of the continuous wave $(\mathrm{CW})$ radars, whereas the second one of the so called pulsed radars. In a $\mathrm{CW}$ radar, the radiated signal is transmitted continuously, whereas, in a pulsed radar, it is sent over short periods of time. In both cases the transmitted signal can be modulated or unmodulated; for this reason, radar systems can be also classified on the basis of the modulating waveform. In the technical literature on vital sign estimation, the use of the following types of radar systems have been investigated: a) CW Doppler radar; b) frequency-modulated continuous wave (FMCW) radar; c) stepped-frequency continuous wave (SFCW) radar; d) impulse radio ultra-wideband (IR-UWB) radar. In the remaining part of this paragraph, a brief description of each type is provided.

Continuous wave Doppler radars radiate a continuous wave radio signal, characterized by a known stable frequency, and are commonly employed for their hardware simplicity. In these radar systems, the chest displacement due to the heart and breathing activities results in a variation of the phase of the received signal. Such a variation is inversely proportional to the wavelength of the signal; therefore, reducing the wavelength of the transmitted wave (i.e., increasing its frequency) results in larger changes in the observed phase and, consequently, allows to detect smaller displacements. One of the main limitations of these radar systems is represented by the fact that they are not able to measure the frontal distance, i.e. the range between the radar and any subject detected by it.

Frequency-modulated continuous-wave radars and stepped-frequency continuous-wave radars radiate wideband frequency modulated signals. The main difference between these systems is represented by the fact that, in the former case, the frequency of the transmitted wave evolves over time in a linear manner, whereas, in the latter one, it changes in a stepwise manner. However, in both systems, the propagation delay is extracted from the phase variations observed in the received signal.

\footnotetext{
${ }^{3}$ The spectral contribution due to respiration is represented by a few relevant harmonics, as evidenced by our numerical results shown in Paragraph VIII.C..
} 
Impulse radio ultra-wideband radars radiate wideband frequency modulated signals. In these systems, the chest distance is estimated by assessing the delay experienced by sub-nanosecond pulses, being this delay proportional to the distance between the radar and any detected subject. Such pulses can be radiated at an high power level if vital signs need to be estimated through walls or behind obstacles.

The above mentioned radar systems can be also classified on the basis of their wavelength or their maximum measurable range. In fact, in the first case, they are divided into micro-wave radars, characterized by a wavelength of few centimetres, and mm-wave radars, if their central frequency is equal to $77 \mathrm{GHz}$ or, in general, greater than $30 \mathrm{GHz}$ [33]. In the second case, instead, they can be divided in: a) short range radars, that are able to measure a maximum range of about $30 \mathrm{~m}$; b) medium range radars, that are characterized by a maximum range of about $100 \mathrm{~m}$; c) long range radars, that achieve the largest maximum range (of the order $250 \mathrm{~m}$ ).

Each of the considered radar systems is endowed with a single antenna or an antenna array at its TX and/or RX sides. A SISO radar system employs a single antenna at both its sides; note that most of the CW and IR-UWB radars considered in technical literature on the monitoring of human vital signs are of SISO type. Multiple-input multiple-output radar systems, instead, employ antenna arrays in their transmission and reception; various FMCW and SFCW radars of this type are already available on the market and their use in vital sign monitoring is currently being investigated. It is also important to keep in mind that a SISO radar can estimate the range and/or the distance of a single/multiple targets, whereas a MIMO radar makes the estimation of its/their angular coordinates possible.

Multiple-input multiple-output radar systems can be divided into statistical (or bistatic) radars [34,35], and colocated radars [36], [37] on the basis of the distance between their transmit and receive arrays. In practice, the transmit and receive antennas of statistical radar systems belonging to the first class are widely separated. On the contrary, the transmit antennas of colocated radar systems are close to the receive ones and, in particular, are usually placed on the same shield. The last feature allows to develop compact devices; this explains why all the MIMO radars currently being considered for vital sign monitoring are of this type.

In a MIMO radar system, the signals radiated by distinct TX antennas are orthogonal. The simplest strategy to synthesize orthogonal waveforms is represented by time division multiplexing (TDM) [38]. Adopting this strategy means that distinct transmit antennas are activated over disjoint time intervals, so that the signals they radiate do not overlap in the time domain.

The most relevant features of the above mentioned radar types are listed in Table 2, where the adjectives low, medium and high are represented by the letters L, M, and H, respectively. For each type, the following distinct technical features are taken into consideration: a) its ability to detect targets at long distance; b) its ability to achieve accurate estimation; c) its ability to detect multiple subjects; d) the simplicity of its architecture (and of the corresponding implementation); e) its cost; f) its robustness, i.e. its ability to operate at a low signal-to-noise ratio (SNR). From the contents of this table it can be easily inferred that FMCW, SFCW and UWB-IR radars are more complicated and costly than CW Doppler radars, but may perform significantly better; in practice, they allow to achieve a good trade-off between cost/complexity and estimation accuracy in vital sign monitoring.

\section{IV.B. Architecture of single-input single-output radar systems}

In this paragraph, a brief description of the architecture of the radar systems employed for vital signs monitoring is provided. All the considered systems are equipped with single TX and RX antenna. Moreover, in illustrating their baseband processing at the receive side, it is always assumed that the chest of the monitored subject, that is placed in front of the radar system, can be represented as a single point target for simplicity ${ }^{4}$.

\section{1) Continuous wave radars}

Let us focus first on CW radar systems. The architecture of a radar system of this type is represented in Fig. 2. In its transmitter, the RF signal produced by a waveform generator (and characterised by

\footnotetext{
${ }^{4}$ As a matter of fact, the chest of the monitored subject is usually much larger than the resolution of the employed radar sensor. For this reason, based on the multiple measurements it generates in response to the radar signal, it should be represented as a cloud of point targets. Note also that all this effects are ignored for the estimation of vital signs.
} 
Table 2: Ranking of the considered types of radar systems in terms of different features.

\begin{tabular}{|c|c|c|c|c|c|}
\hline Features & & CW & FMCW & SFCW & UWB-IR \\
\hline \multirow{2}{*}{$\begin{array}{c}\text { Long distance } \\
\text { detection }\end{array}$} & SISO & $\mathrm{L}$ & $\mathrm{M}$ & $\mathrm{M}$ & M \\
\hline & MIMO & $\mathrm{L}$ & $\mathrm{H}$ & $\mathrm{H}$ & $\mathrm{H}$ \\
\hline \multirow{2}{*}{ Accuracy } & SISO & $\mathrm{L}$ & $\mathrm{M}$ & $\mathrm{M}$ & M \\
\hline & MIMO & $\mathrm{L}$ & $\mathrm{H}$ & $\mathrm{H}$ & $\mathrm{H}$ \\
\hline \multirow{2}{*}{$\begin{array}{c}\text { Detection of } \\
\text { multiple subjects }\end{array}$} & SISO & $\mathrm{L}$ & $\mathrm{M}$ & $\mathrm{M}$ & M \\
\hline & MIMO & M & $\mathrm{H}$ & $\mathrm{H}$ & $\mathrm{H}$ \\
\hline \multirow{2}{*}{ Simplicity } & SISO & $\mathrm{H}$ & M & $\mathrm{L}$ & M \\
\hline & MIMO & M & $\mathrm{L}$ & $\mathrm{L}$ & $\mathrm{L}$ \\
\hline \multirow{2}{*}{ Cost } & SISO & $\mathrm{L}$ & $\mathrm{M}$ & $\mathrm{M}$ & $\mathrm{M}$ \\
\hline & MIMO & $\mathrm{L}$ & $\mathrm{H}$ & $\mathrm{H}$ & $\mathrm{H}$ \\
\hline \multirow{2}{*}{ Robustness } & SISO & $\mathrm{L}$ & M & M & $\mathrm{M}$ \\
\hline & MIMO & M & $\mathrm{H}$ & $\mathrm{H}$ & $\mathrm{H}$ \\
\hline
\end{tabular}

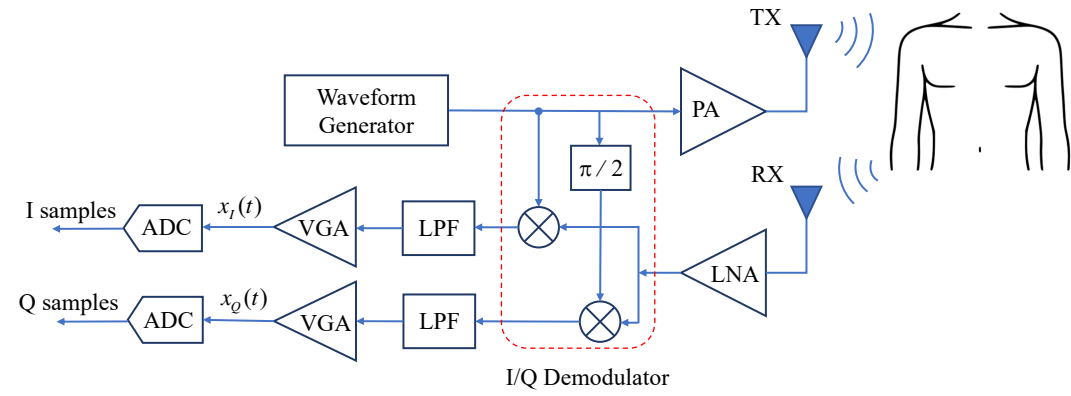

Figure 2: Block diagram of a CW radar.

the carrier frequency $f_{0}$ ) feeds a power amplifier (PA), whose response is applied to the TX antenna; this antenna is placed in front of the chest of a human being. The received signal is amplified by a low noise amplifier (LNA), whose response undergoes frequency downconversion to extract its in-phase and quadrature components, denoted $x_{I}(t)$ and $x_{Q}(t)$, respectively; this task is accomplished by a couple of mixers, each followed by the cascade of a low-pass filter (LPF) with a variable gain amplifier (VGA). The output of each VGA is sampled by an analog-to-digital converter (ADC), operating at the frequency $f_{s}=1 / T_{s}$, where $T_{s}$ is the sampling period. The $n$-th sample of $x_{I}(t)\left(x_{Q}(t)\right)$ is denoted $x_{I}[n] \triangleq x_{I}\left(n T_{s}\right)\left(x_{Q}[n] \triangleq x_{Q}\left(n T_{s}\right)\right)$; note that its expression depends on the transmitted waveform. Further mathematical details are provided in the following for each of the three types of CW radars introduced in Paragraph IV.A..

Continuous wave Doppler radar In this case, the waveform generator appearing in Fig. 2 consists in a local oscillator (LO) generating a tone at the frequency $f_{c}$. The $n$-th sample of $x_{I}(t)$ and $x_{Q}(t)$ can be expressed as (e.g., see [39, Sect. II, eqs. (1)-(2)])

$$
x_{I}[n]=a \cos (\psi[n])+w_{I}[n]
$$

and

$$
x_{Q}[n]=a \sin (\psi[n])+w_{Q}[n],
$$

respectively, with $n=0,1, \ldots, N-1$; here, $n$ is the fast time index, $N$ represents the overall number of samples acquired in the considered observation interval, $a$ represents the amplitude of the useful signal 
component, $w_{I}[n]\left(w_{Q}[n]\right)$ is the contribution of the additive white Gaussian noise (AWGN) affecting the in-phase (quadrature) component,

$$
\begin{gathered}
\psi[n] \triangleq \psi_{0}+\Delta \psi[n], \\
\psi_{0}=4 \pi \frac{R_{0}}{\lambda}
\end{gathered}
$$

is a constant phase shift due to the (fixed) distance $R_{0}$ between the chest of the considered subject and the $\operatorname{radar}^{5}$, and

$$
\Delta \psi[n]=\frac{4 \pi}{\lambda} \Delta R[n]
$$

is the phase variation due to the chest displacement $\Delta R(t)$ (see (1)); here, $\Delta R[n] \triangleq \Delta R\left(t=n T_{s}\right), \lambda=f_{0} / c$ is the wavelength of the radiated signal and $c$ is the speed of light. From the mathematical results illustrated above it can be easily inferred that the chest displacement can be assessed by estimating the phase variations over consecutive samples of the complex sequence $\{x[n] ; n=0,1, \ldots, N-1\}$, where

$$
x[n] \triangleq x_{I}[n]+j x_{Q}[n]=a \exp (j \psi[n])+w[n],
$$

and

$$
w[n] \triangleq w_{I}[n]+j w_{Q}[n]
$$

is the noise contribution to $x[n](14)$.

Frequency modulated continuous wave radar The waveform generator employed in the transmitter of an FMCW radar (and appearing in Fig. 2) consists in a voltage controlled oscillator (VCO), characterised by the free running frequency $f_{0}$ and fed by a periodic ramp generator. The frequency of the transmitted signal evolves periodically and, within each period, changes linearly; this linear frequency sweep is known as chirp. The evolution of the instantaneous frequency over a frame consisting of $N_{c}$ consecutive chirps is shown in Fig. 3 ; here $T_{0}, T$ and $T_{R}$ represent the chirp duration, the ramp time period and the reset time, respectively, so that

$$
T_{0}=T+T_{R},
$$

and the overall duration of the frame is $T_{F}=N_{c} T_{0}$. Note that each chirp is characterised by the chirp slope $\mu=B / T$, where $B$ is the width of the swept frequency interval (i.e., the radar bandwidth).

The $k$-th sample of the in-phase and quadrature components acquired in the $n$-th chirp interval can be expressed as (e.g., see [40, Sect. 2.1.2, eq. (5)])

$$
x_{I}[k, n]=a \cos \left(2 \pi k f_{n} T_{s}+\psi[n]\right)+w_{I}[k, n]
$$

and

$$
x_{Q}[k, n]=a \sin \left(2 \pi k f_{n} T_{s}+\psi[n]\right)+w_{Q}[k, n],
$$

respectively, with $k=0,1, \ldots, N-1$ and $n=0,1, \ldots, N_{c}-1$; here, $k(n)$ denotes the fast time (slow time) index, $w_{I}[k, n]\left(w_{Q}[k, n]\right)$ is the contribution of the AWGN affecting the in-phase (quadrature) component of the useful signal, $a$ represents the amplitude of the useful signal component,

$$
f_{n} \triangleq \mu \tau_{n}
$$

is a frequency proportional to the delay

$$
\tau_{n} \triangleq \frac{2 R_{n}}{c}
$$

$R_{n}$ is the distance of the radar system from the chest (i.e., the target range) in the $n$-th chirp interval. Note that the phase term $\psi[n]$ appearing in the right hand side (RHS) of (17) and (18) is assumed constant within a single chirp and, therefore, it is still expressed by (11).

\footnotetext{
${ }^{5}$ The phase shift $\psi_{0}$ is called direct current (DC) offset. In vital sign monitoring, this quantity depends on the distance between the employed radar and the chest wall in front of it; however, this term may be influenced by other factors, such as the reflections from stationary targets or from other parts of the human body, and the noise of electronic components.
} 


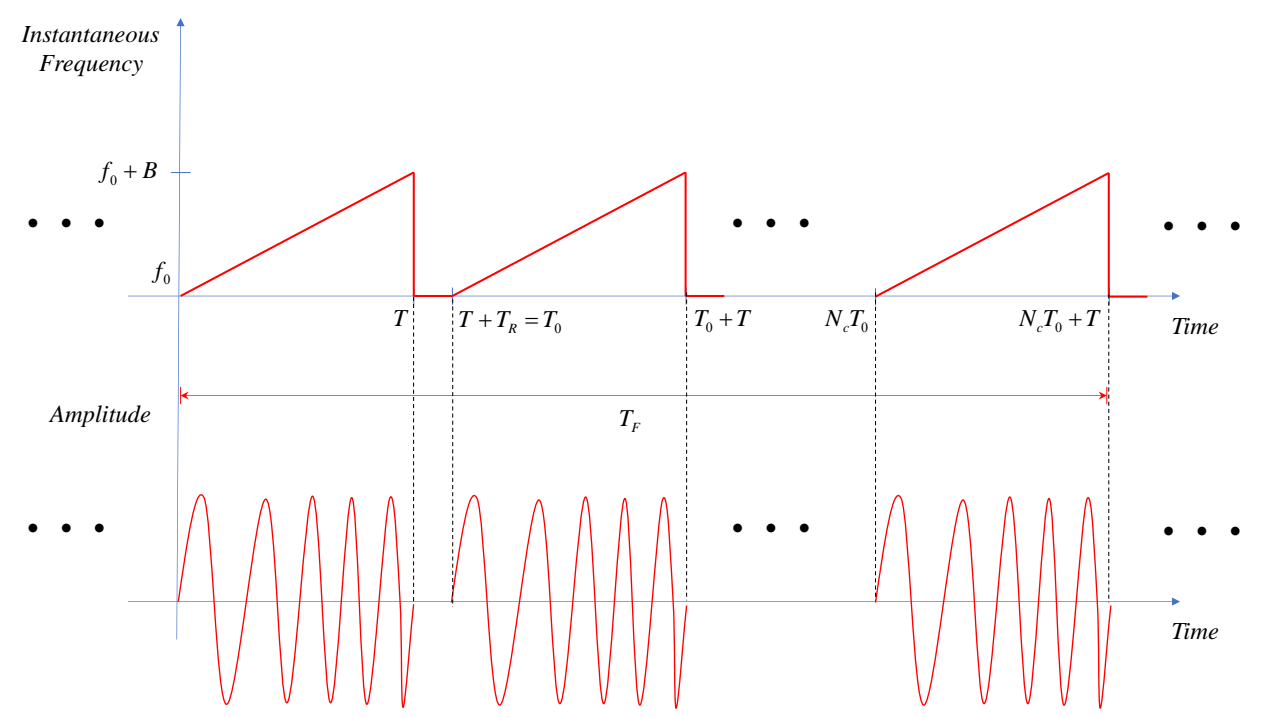

Figure 3: Representation of the instantaneous frequency of the RF signal transmitted in an FMCW radar system.

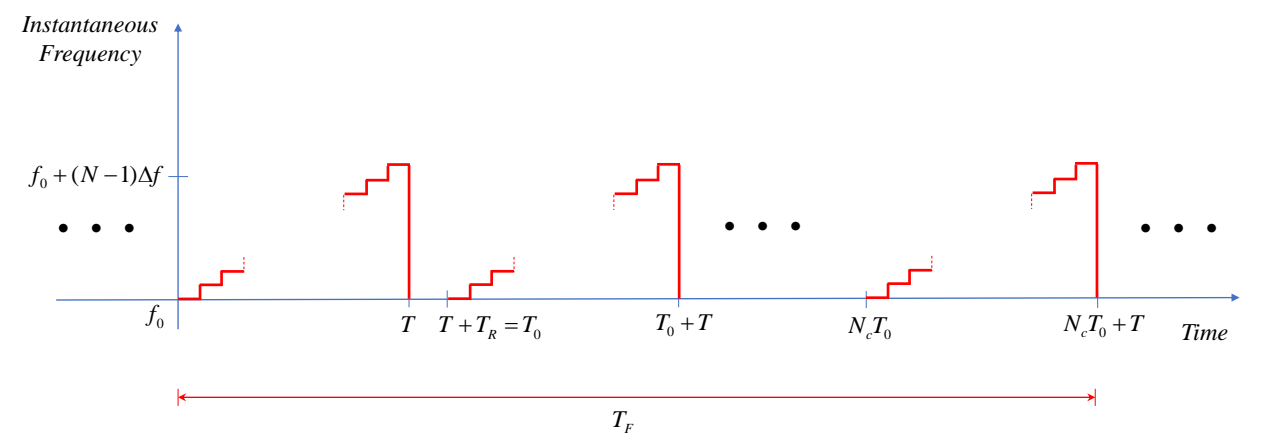

Figure 4: Representation of the instantaneous frequency of the RF signal transmitted by an SFCW radar system.

In the considered radar system, the complex sequence

$$
\begin{aligned}
x[k, n] & \triangleq x_{I}[k, n]+j x_{Q}[k, n] \\
& =a \exp \left(j\left(2 \pi k f_{n} T_{s}+\psi[n]\right)\right)+w[k, n],
\end{aligned}
$$

is processed to generate an estimate of the frequency $f_{n}$, from which an estimate of the target range $R_{n}$ is easily computed on the basis of (19)-(20); here, $w[k, n] \triangleq w_{I}[k, n]+j w_{Q}[k, n]$. Consequently, range estimation is equivalent to the estimation of the normalised frequency

$$
F_{n} \triangleq f_{n} T_{s}
$$

of a complex exponential sequence.

Stepped frequency continuous wave radar The transmitter of an SFCW radar is similar to that of an FMCW radar, the only difference being represented by the fact that the ramp generator of the last system is replaced by a staircase waveform generator. Therefore, the instantaneous frequency of the signal generated by the VCO employed in an SFCW radar changes in a step-wise manner within each radiated frequency sweep. The time evolution of the instantaneous frequency of the signal generated by the VCO over a single frame is shown in Fig. 4. In this figure, $T_{0}, T$ and $T_{R}$ represent the frequency sweep duration, the sampling time and the reset time, respectively, whereas $N$ and $\Delta f$ represents the overall number and the width of 
each frequency step, respectively. Note that, if $N_{c}$ denotes the overall number of frequency sweeps forming a single frame, each frame lasts $T_{F}=N_{c} T_{0}$ s.

If we assume that the sampling interval $T_{s}$ is equal to the duration of each frequency step (i.e., that the sampling frequency $f_{s}=1 / T_{s}$ is equal to $\Delta f$ ), a single complex sample is acquired at the RX side within each single frequency step. Moreover, in this case, the $k$-th sample of the in-phase and quadrature components available in the $n$-th frequency sweep interval can be expressed as (e.g., see [41, Sect. II.B, eq. (13)])

$$
x_{I}[k, n]=a \cos \left(2 \pi k \Delta f \tau_{n}+\psi[n]\right)+w_{I}[k, n]
$$

and

$$
x_{Q}[k, n]=-a \sin \left(2 \pi k \Delta f \tau_{n}+\psi[n]\right)+w_{Q}[k, n],
$$

respectively; here, $k, n, a, \psi[n], \tau_{n}, w_{I}[k, n], w_{Q}[k, n]$ and $N$ have the same meaning as the one illustrated for the corresponding terms appearing in (17)-(18).

In the considered radar system, the complex sequence $\{x[k, n] ; k=0,1, \ldots, N-1\}$, where

$$
\begin{aligned}
x[k, n] & \triangleq x_{I}[k, n]+j x_{Q}[k, n] \\
& =a \exp \left(-j\left(2 \pi k \Delta f \tau_{n}+\psi[n]\right)\right)+w[k, n]
\end{aligned}
$$

for any $n$, is processed to generate an estimate of the normalised delay

$$
F_{n} \triangleq \Delta f \tau_{n}
$$

Since the last quantity can be also interpreted as the normalised frequency of a complex exponential sequence, the frequency estimation algorithms developed for FMCW radar systems can be also employed in SFCW systems; in doing so, we must keep in mind that the only difference between (21) and (25) is represented by the sign of the argument of complex exponential appearing in their RHSs. Finally, it is worth stressing that this similarity can be considered as a form of time-frequency duality [42]; from this viewpoint, an SFCW radar system can be seen as the dual of an FMCW radar system, as evidenced in [43].

\section{2) Impulse radio ultra-wideband radar}

The architecture of an IR-UWB radar is represented in Fig. 5. At the TX side, a square wave generator (SWG) is used to trigger a tunable Gaussian pulse generator, whose (baseband) output signal $s(t)$ can be expressed as

$$
s(t)=\sum_{n} p\left(t-n T_{0}\right)
$$

here, $p\left(t-n T_{0}\right)$ represents the $n$-th transmitted pulse and $T_{0}$ is the pulse repetition interval (PRI). Note that, if $T$ denotes the duration of each pulse ${ }^{6}$, the interval $T_{0}$ is expressed by (16), where $T_{R}$ is the so called reset time. The time evolution of $s(t)$ is exemplified by Fig. 6, where $N_{c}$ consecutive pulses are represented; such pulses form a transmission frame, whose duration is $T_{F}=N_{c} T_{0}$ s.

The signal $s(t)(27)$ undergoes frequency upconversion (accomplished by means of a mixer and a LO operating at the frequency $\left.{ }^{7} f_{0}\right)$ and power amplification before its transmission.

Since the pulses forming $s(t)(27)$ are not overlapped, we can focus our attention on the echo generated by the chest in response to the $k$-th pulse. At the RX side, the RF signal conveying this pulse is amplified by an LNA and downconverted ${ }^{8}$ to extract its in-phase and quadrature components. Then, these components undergo analog-to-digital conversion at the frequency $f_{s}=1 / T_{s}$, where $T_{s}$ is the sampling period. If $\tau_{n}$ denotes the delay experienced by the considered pulse, the $k$-th sample of the in-phase and quadrature components associated with it can be expressed as (e.g., see [44, Sect. II, eq. (4)])

$$
x_{I}[k, n]=a p[k, n] \cos (\psi[n])+w_{I}[k, n]
$$

and

$$
\left.x_{Q}[k, n]=a p[k, n] \sin (\psi[n]]\right)+w_{Q}[k, n],
$$

\footnotetext{
${ }^{6}$ In practice, $T$ is on the order of hundreds of ps;

${ }^{7}$ This frequency usually belongs to the industrial, scientific and medical (ISM) band.

${ }^{8}$ The downconversion scheme is the same as that illustrated in Fig. 2.
} 


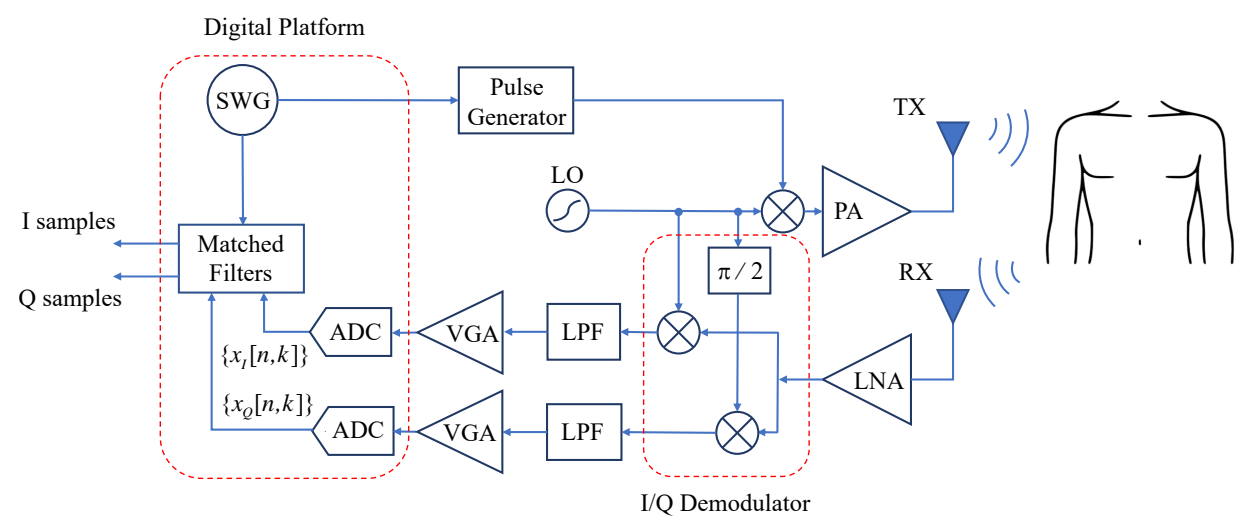

Figure 5: Block diagram of an IR-UWB radar.

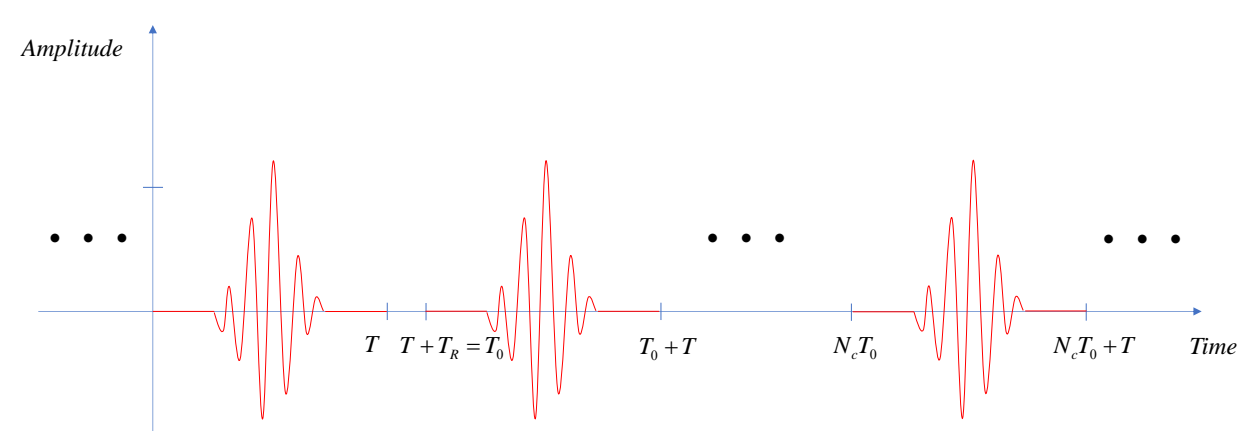

Figure 6: Example of the baseband signal $s(t)(27)$ produced by the pulse generator of the IR-UWB radar system represented in Fig. 5.

Table 3: Received signal model and range formulas for the considered radar systems.

\begin{tabular}{clll}
\hline Radar type & Waveform & Received signal model & Target range \\
\hline \multirow{3}{*}{ Continuous wave } & Sine wave & $x[n]=a \exp (j \psi[n])$ & unavailable \\
& Linear frequency modulation & $x[k, n]=a \exp \left(j\left(2 \pi k f_{n} T_{s}+\psi[n]\right)\right)$ & $R_{n}=f_{n} \cdot c /(2 \mu)$ \\
& Stepped frequency modulation & $x[k, n]=a \exp \left(-j\left(2 \pi k \Delta f \tau_{n}+\psi[n]\right)\right)$ & $R_{n}=\tau_{n} \cdot c / 2$ \\
\hline Impulse-radio & Gaussian pulse & $x[k, n]=a p[k, n] \exp (j \psi[n])$ & $R_{n}=\tau_{n} \cdot c / 2$ \\
\hline
\end{tabular}
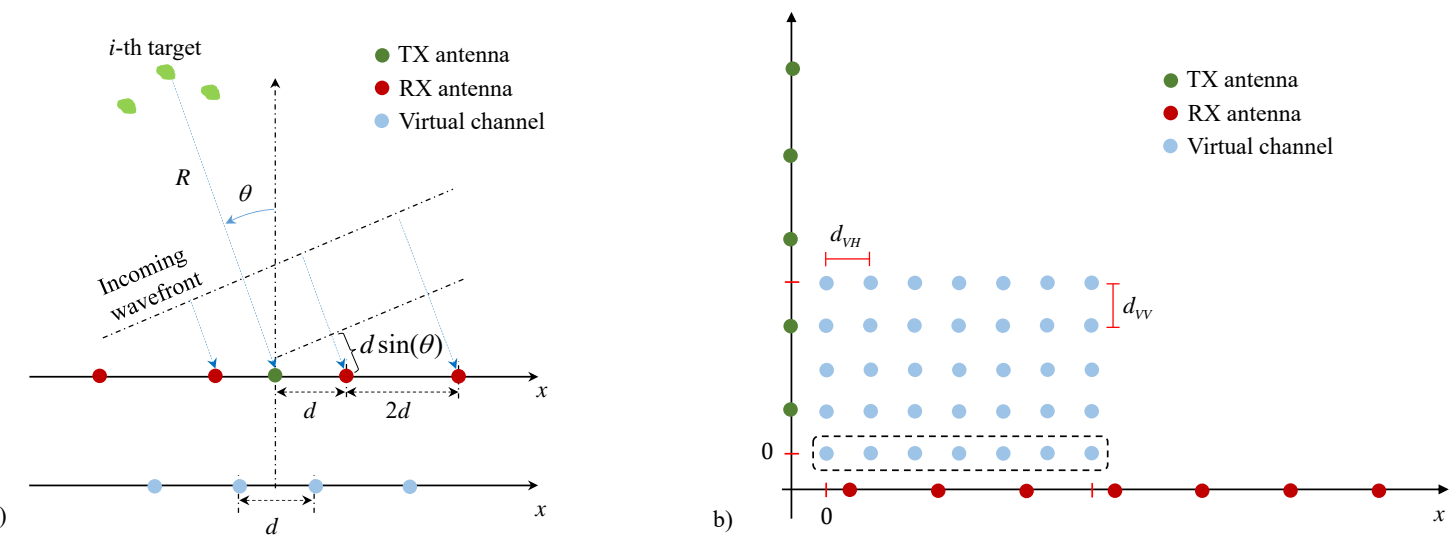

Figure 7: Representation of: a) an ULA; b) an URA. In both cases the physical array and the associated virtual array are considered. 
respectively, with $k=0,1, \ldots, N-1$ and $n=0,1, \ldots, N_{c}-1$; here, $a$ represents the amplitude of the useful signal component,

$$
p[k, n] \triangleq p\left(k T_{s}-\tau_{n}-n T_{0}\right),
$$

$n, k, \psi[n]$ have the same meaning as the one illustrated for the corresponding terms appearing in (17)(18), $w_{I}[k, n]$ and $w_{Q}[k, n]$ represent noise contributions (they correspond to the samples $w_{I}[n]$ and $w_{Q}[n]$ contained in the RHS of (9) and (13), respectively), and $N$ is the overall number of samples acquired over each PRI (briefly, of fast time samples). Each of the sequences $\left\{x_{I}[k, n]\right\}$ and $\left\{x_{Q}[k, n]\right\}$ feeds a digital matched filter (MF), that is a digital filter whose impulse response is the sequence $\left\{p\left(T-k T_{s}\right) ; k=0,1\right.$, ..., $N-1\}$; the responses of the MFs fed by the in-phase and quadrature components are denoted $\left\{\hat{x}_{I}[k, n]\right.$; $k=0,1, \ldots, N-1\}$ and $\left\{\hat{x}_{Q}[k, n] ; k=0,1, \ldots, N-1\right\}$, respectively. If the chest displacement $\Delta R(t)$ is approximately constant within a single PRI, i.e.

$$
\Delta R\left(k T_{s}+n T_{0}\right) \cong \Delta R\left(n T_{0}\right)
$$

for $k=0,1, \ldots, N-1$ and any $n$, matched filtering allows to maximise the output SNR, i.e. the ratio between the energy of the useful component available at the MF output and the average power of the noise component affecting it. The target delay $\tau_{n}$ is estimated by identifying the position of the main peak appearing in the MF response.

Finally, it is useful to point out that (28)-(29) can be condensed in the complex signal model

$$
\begin{aligned}
x[k, n] & \triangleq x_{I}[k, n]+j x_{Q}[k, n] \\
& =a p[k, n] \exp (j \psi[n])+w[k, n],
\end{aligned}
$$

where $w[k, n] \triangleq w_{I}[k, n]+j w_{Q}[k, n]$ is the noise component. In an UWB-IR radar systems, the presence of clutter, i.e. of reflections from other stationary targets, may affect the estimation of vital signs. The presence of clutter in the received signal can be modelled as DC offset or small amplitude variations in the slow time dimension in (28) and (29).

The received signal models and range formulas provided above for the different types of radar systems are summarized in Table 3.

\section{IV.C. Architecture of multiple-input multiple-output radar systems}

A MIMO radar system is equipped with a TX and an RX antenna array, consisting of $N_{T}$ and $N_{R}$ elements, respectively. In a colocated MIMO radar, TX antennas are close to the RX ones and are usually placed on the same shield. It is important to keep in mind that:

a) The elements of the TX array are employed to radiate orthogonal waveforms, whereas the RX elements to receive distinct replicas of the electromagnetic echoes generated by multiple targets.

b) In these conditions, any couple of physical TX and RX antennas generates independent measurements; for this reason, each of the

$$
N_{V} \triangleq N_{T} \cdot N_{R}
$$

couples of TX/RX antennas can be replaced by a single virtual antenna (VA) of an equivalent SISO radar. The abscissa $x_{v}$ and the ordinate $y_{v}$ of the $v$-th VA element associated with the $p$-th TX antenna and the $q$-th RX antenna (briefly, the $(p, q)$ VA) are computed as (e.g., see [45, Par. II.A, eq. (1)])

$$
x_{v}=\frac{x_{p}+x_{q}}{2}
$$

and

$$
y_{v}=\frac{y_{p}+y_{q}}{2}
$$

respectively, with $p=0,1, \ldots, N_{T}-1, q=0,1, \ldots, N_{R}-1$ and $v=0,1, \ldots, N_{V}-1$; thus, a physical array characterized by $N_{T}\left(N_{R}\right)$ TX $(\mathrm{RX})$ elements is equivalent to a virtual array constituted by $N_{V}$ channels. 
Two physical arrays and the associated virtual arrays are shown in Figs. 7-a) and b). More specifically, in Fig. 7-a), the one-dimensional (1D) virtual array associated with the given physical array is a uniform linear array (ULA), since it consists of $N_{V}=1 \cdot 4=4$ equally spaced and aligned virtual elements (see (33)); note that, in this case, $y_{v}=0$ (see (35)), because of the geometry of the physical array and the selected reference systems, and that the distance between two adjacent VAs is equal to $d$. Putting together multiple parallel ULAs leads to the 2D uniform rectangular array (URA), like the one shown in Fig. 7-b). The last array is made of $N_{V}=5 \cdot 7=35 \mathrm{VAs}$ (see (33)) or, equivalently, of $N_{V U L A}=5\left(N_{H U L A}=7\right)$ horizontal (vertical) ULAs (one of them is highlighted by a black dashed rectangle), each composed by seven (five) VAs. Note also that, in Fig. 7, the distance between two adjacent horizontally-aligned (vertically-aligned) VAs is denoted $d_{\mathrm{VH}}\left(d_{\mathrm{VV}}\right)$ and that $d_{\mathrm{VH}}=d_{\mathrm{VV}}$ is assumed. The distance between two adjacent physical elements is usually equal to $\lambda / 2$, where $\lambda$ is the wavelength characterizing the radiated waveforms; consequently, the distances $d, d_{\mathrm{VV}}$ and $d_{\mathrm{VH}}$ are all equal to $\lambda / 4$.

Let us focus now on a radar system employing the ULA represented in Fig. 7-a) and assume that: a) $L$ point targets are detected by this system; b) the position of the $i$-th target (with $i=0,1, \ldots, L-1$ ) can be deemed constant in the frame interval in which it is detected (i.e., over an interval lasting $T_{F} \mathrm{~s}$ ). In this case, the useful component of the received signal consists of the superposition of $L$ contributions, each associated with a different target. More specifically, the contribution given by $i$-th target is given by

$$
x_{i}^{(v)}[n]=a_{i}^{(v)} \exp \left(j \psi_{i}^{(v)}[n]\right),
$$

for the CW radar system described in paragraph 1) (see (14)),

$$
x_{i}^{(v)}[k, n]=a_{i}^{(v)} \exp \left(j\left(2 \pi k f_{i}^{(v)} T_{s}+\psi_{i}^{(v)}[n]\right)\right),
$$

for the FMCW radar system described in Paragraph 1) (see (21)),

$$
x_{i}^{(v)}[k, n]=a_{i}^{(v)} \exp \left(-j\left(2 \pi k \tau_{i}^{(v)} \Delta f+\psi_{i}^{(v)}[n]\right)\right),
$$

for the SFCW radar system described in Paragraph 1) (see (25)) and

$$
x_{i}^{(v)}[k, n]=a_{i}^{(v)} p\left(k T_{s}-\tau_{i}^{(v)}-n T_{0}\right) \exp \left(j \psi_{i}^{(v)}[n]\right)
$$

for the IR-UWB radar system described in Paragraph 2) (see (32)). In (36)-(39) it is assumed that:

a) The integer $v$ denotes the index of the selected VA (we assume that $v=0,1, \ldots, N_{V}-1$ ).

b) The positive real $a_{i}^{(v)}$ is the amplitude of the contribution due to the $i$-th target and observed on the $v$-th VA.

c) The phase $\psi_{i}^{(v)}[n]$ can be expressed as

$$
\psi_{i}^{(v)}[n]=\psi_{0, i}^{(v)}+\Delta \psi_{i}[n]
$$

where

$$
\psi_{0, i}^{(v)}=\frac{4 \pi}{\lambda}\left(R_{0, i}+v d \sin \left(\theta_{i}\right)\right),
$$

$\Delta \psi_{i}[n]$ is still expressed by (13), $\theta_{i}$ is the azimuth of the $i$-th target and $R_{0, i}$ is the range of the $i$-th target assuming zero chest displacement.

d) The frequency $f_{i}^{(v)}$ is given by

$$
f_{i}^{(v)}=\mu \tau_{i}^{(v)}
$$

whereas the delay $\tau_{i}^{(v)}$ can be approximated as

$$
\tau_{i}^{(v)} \approx \frac{2}{c}\left(R_{0, i}+v d \sin \left(\theta_{i}\right)\right),
$$

if we assume that $R_{0, i}$ is much larger that the maximum range variations $\Delta R(t)$ experienced by the $i$-th target in the considered frame interval (see (1 in Par. III.B.). 
Finally, it is important to point out that, in CW and IR-UWB radar systems, the azimuth of a given target influences the phase of the received signal component associated with it, whereas in FMCW (SFCW) radar systems, it influences also the normalised frequency (normalised delay) characterising that component. However, in any case, an estimate of the direction of arrival (DOA) of the electromagnetic echo originating from a given target is computed on the basis of the phases referring to that target and observed over multiple antennas.

\section{Signal Processing Algorithms for Vital Sign Monitoring}

In this section, we first describe the most important deterministic and learning-based processing methods that can be employed to extract vital signs from the measurements provided by the SISO radar systems described in the previous section. Then, we provide essential information about the processing accomplished in colocated FMCW and SFCW MIMO radars for estimating the vital signs of multiple people. Finally, we illustrate some numerical results generated by applying some of the considered methods to a synthetically generated dataset.

\section{V.A. Deterministic detection and estimation algorithms for single-input single- output radars}

The majority of the radar-based methods for vital sign monitoring appeared in literature have the following features: a) they are deterministic (i.e., model-based), since their derivation is based on our prior knowledge about the structure of radar echoes; b) they extract vital signs from the phase of the received signal. As far as the last point is concerned, it is useful to focus first on a SISO CW Doppler radar for simplicity and to reconsider the phase expression

$$
\psi[n] \triangleq \psi_{0}+\Delta \psi[n]
$$

provided in Paragraph 1) for the $n$-th received signal sample (where $n$ denotes the fast time index). As already illustrated in that paragraph, the constant $\psi_{0}$ appearing in the last equation represents the so called DC offset, whereas (see (13))

$$
\Delta \psi[n]=\frac{4 \pi}{\lambda} \Delta R[n]
$$

is a time-varying term related to the body movement induced by breath and cardiac activities and, consequently, conveys the information we are interested in. This explains why the first step accomplished by the deterministic methods developed for CW Doppler radars consists in extracting the phase of the received signal sequence $\{x[n]\}$, as shown in Fig. 8, where a block diagram is represented to describe the overall processing they accomplish. As shown in this figure, phase extraction is carried out by the first block, that generates the $N_{r}$-dimensional vector

$$
\hat{\psi} \triangleq\left[\hat{\psi}[0], \hat{\psi}[1], \ldots, \hat{\psi}\left[N_{r}-1\right]\right]^{T}
$$

on the basis of $\{x[n]\}$; here, $N_{r}$ is the overall number of measurements and $\hat{\psi}[n]$ represents an estimate of $\psi[n]$ (44), i.e. of the phase of the complex sample $x[n]$ (which is expressed by (14) in the case of a single point target). Given the vector $\hat{\boldsymbol{\psi}}$, estimates of the BR and of the HR can be evaluated by applying the so called periodogram method [46], i.e. by identifying dominant frequency components in the amplitude spectrum of $\{\hat{\psi}[n]\}$. In fact, it is known that: a) the highest peak in the above mentioned spectrum is found at the breath frequency in normal respiration conditions; b) the HR is higher than the BR (at least more than two times higher). For these reasons, an estimate $\hat{f}_{b}$ of the BR $f_{b}$ (expressed in acts/s) can be computed as

$$
\hat{f}_{b}=\hat{b} f_{r}
$$

where

$$
\begin{gathered}
\hat{b}=\arg \max _{\tilde{b} \in\left\{0,1, \ldots, N_{0} / 2\right\}}\left|Y_{\tilde{b}}\right|, \\
Y_{\tilde{b}} \triangleq \frac{1}{N_{r}} \sum_{n=0}^{N_{r}-1} \hat{\psi}[n] \exp \left(-j 2 \pi n \tilde{b} / N_{0}\right),
\end{gathered}
$$


$M$ is the oversampling factor and

$$
N_{0} \triangleq M N_{r}
$$

$$
f_{r} \triangleq \frac{1}{N_{0} T_{s}}
$$

for the CW Doppler radar system described in Paragraph IV.B.. Note that: a) $Y_{\tilde{b}}$ (49) represents the $\tilde{b}$-th element of an $N_{0}$ order Discrete Fourier Transform (DFT) of $\hat{\boldsymbol{\psi}}(46)$ and can be efficiently computed by adopting a Fast Fourier Transform (FFT) algorithm of the same order, as shown in Fig. 8; b) the stragey expressed by (48) aims at identifying the dominant spectral component in the spectrum of the sequence $\{\hat{\psi}[n]\}$.

A similar procedure can be employed for estimating the $\mathrm{HR} f_{h}$. However, in this case, FFT processing is preceded by bandpass filtering (BPF) to cancel all the spectral components whose frequency falls outside the interval in which the heart frequency is expected (see Fig. 8). Then, an estimate $\hat{f}_{h}$ of $f_{h}$ can be evaluated as

$$
\hat{f}_{h}=\hat{h} \bar{f}_{r}
$$

where

$$
\hat{h}=\arg \bar{f}_{\tilde{h} \in\left\{0,1, \ldots, \bar{N}_{0} / 2\right\}} \max _{\bar{N}_{0} T_{s}}\left|Z_{\tilde{h}}\right|
$$

and $Z_{\tilde{h}}(49)$ represents the $\tilde{h}$-th element of an

$$
\bar{N}_{0} \triangleq \bar{M} N_{r}
$$

order DFT of the BPF output vector, having size of length $N_{r}$ ( $\bar{M}$ denotes the adopted oversampling factor). It is worth pointing out that:

a) Since some prior knowledge about the minimum and maximum BRs to be detected is usually available, the search interval in the RHS of (48) can be restricted to reduce the overall computational cost of the search for the maximum over the set $\left\{\left|Y_{\tilde{b}}\right|\right\}$. Similar considerations hold for (54), since $\left\{\left|Z_{\tilde{h}}\right|\right\}$ takes on significant values in a restricted frequency range because of the employed bandpass filtering.

b) Various options are available for the bandpass filter topology and order, depending on the required selectivity. However, the main problem is represented by the selection of its passband, since we must avoid that the BR (HR) falls inside (out of) it. In general, the lower limit $f_{L}$ and upper limit $f_{U}$ of its passband should be selected in a way that $f_{L} \geq 2 / \tilde{T}_{\mathrm{BR}}$ and $f_{U} \leq 3 / \tilde{T}_{\mathrm{HR}}$, where $T_{\mathrm{BR}}\left(T_{\mathrm{HR}}\right)$ is the breath (heart) period expressed in seconds. Typical values of $\tilde{T}_{\mathrm{BR}}\left(\tilde{T}_{\mathrm{HR}}\right)$ at rest are given in Par. III.A..

c) Generally speaking, the evaluation of an FFT of order $N_{0}\left(\bar{N}_{0}\right)$ in BR (HR) estimation leads to partitioning the frequency interval in $N_{0}\left(\bar{N}_{0}\right)$ frequency bins, all having the same size. The quantity $\hat{b}(48)(\hat{h}(54))$ represents an estimate of the index of frequency bin inside which the fundamental frequency of respiration (heart) signal falls, whereas $f_{r}(51)\left(\bar{f}_{r}(53)\right)$ is the bin size. Reducing this size (i.e., increasing the FFT order) improves the resolution in frequency estimation at the price of an higher computational load.

d) Since the phase vector $\hat{\boldsymbol{\psi}}$ is real, the FFT outupt vectors $\mathbf{Y}$ and $\mathbf{Z}$ are Hermitian symmetric; this explains why only a portion of its elements is involved in the search required by (48) and (54), respectively.

Let us focus now on the extraction of phase information in CW Doppler radars. This is usually accomplished by means of the arctangent demodulation (AD) technique. This means that the $n$-th element of the vector $\hat{\boldsymbol{\psi}}(46)$ is evaluated as ${ }^{9}$

$$
\hat{\psi}[n]=\arctan \left(\frac{x_{Q}[n]}{x_{I}[n]}\right),
$$

\footnotetext{
${ }^{9}$ The $\arctan (\cdot)$ operator can be also replaced by the $\arctan 2(\cdot)$ operator in order to extend the codomain from $(-\pi / 2, \pi / 2)$ to $(-\pi, \pi)$.
} 
for $n=0,1, \ldots, N_{r}-1$. If $x_{I}[n]$ or $x_{Q}[n]$ are equal to zero, the last equation is replaced by

$$
\hat{\psi}[n]=\arcsin \left(x_{Q}[n]\right)
$$

and

$$
\hat{\psi}[n]=\arccos \left(x_{I}[n]\right)
$$

respectively. It is important to point out that:

a) The contribution of DC offset and strong low frequency components to the received signal phase can be mitigated by exploiting the method illustrated in [47] and based on polynomial fitting. This method allows to capture the slow temporal variations of that phase and to subtract them from it. Other techniques for DC offset removal have been proposed in [48], and are based on a least-square (LS) approach and Kalman filtering.

b) The estimated phase sequence $\{\hat{\psi}[n]\}$ may exhibit some discontinuities. In fact, a discontinuity appears in the extracted phase whenever the condition ${ }^{10}$ (see eq. (45))

$$
\Delta \hat{\psi}_{n+1, n} \triangleq|\hat{\psi}[n+1]-\hat{\psi}[n]|=\frac{4 \pi}{\lambda} \Delta R_{n+1, n} \geq 2 \pi
$$

is met; here, $\Delta R_{n+1, n} \triangleq|\hat{R}[n+1]-\hat{R}[n]|$. This means that, whenever the range variation $\Delta R_{n+1, n}$ observed over two consecutive sampling epochs exceeds half a wavelength, the value of the estimated phase becomes ambiguous. Note that this problem becomes more relevant as the frequency $f_{0}$ of the radiated signal increases (for instance, if $f_{0}=77 \mathrm{GHz}$, any displacement exceeding $2 \mathrm{~mm}$ produces a phase ambiguity).

c) The phase sequence $\{\hat{\psi}[n]\}$ always undergoes a transformation known as unwrapping; unwrapping aims at ensuring that the variation between two consecutive elements of this sequence does not exceed $\pi$. In practice, this result is achieved by adding a multiple of $2 \pi$ to some of the elements of the sequence $\{\hat{\psi}[n]\}$. However, the use of unwrapping may introduce errors in the presence of abrupt phase variations. In this case, the extended differentiate and cross-multiply (DACM) algorithm proposed in [49] should be employed to achieve precise phase unwrapping.

Finally, it is worth mentioning that an alternative to the arctangent method is represented by the complex signal demodulation (CSD) technique. This technique is based on the idea that the received signal can be seen as a frequency modulated waveform (see eq. (6) and (45)). Therefore, an approximate model, based on the first order Bessel functions, can be derived for it [50]. This allows to separate the contribution due to the periodic movement of the chest from the one associated with the position of the body (i.e., with the term $\psi_{0}$; see (12)). In particular, Fourier analysis can be directly applied to the sequence $\{x[n]\}$ (see (14)) to estimate both BR and HR.

The architecture of the deterministic methods developed for FMCW, SFWC and IR-UWB radars can be also represented through the block diagram in Fig. 8. However, in these cases, the following changes are made:

a) The parameter $T_{s}$ appearing in the RHSs of (51) and (53) is replaced by the PRI $T_{p}$ in the case of IR-UWB radar system and by chirp (frequency sweep) duration $T_{0}$ in the case of an FMCW (SFCW) radar system.

b) The algorithm for extracting phase estimation (i.e., for generating the vector $\hat{\boldsymbol{\psi}}(46)$ ) is more complicated.

As far as the last point is concerned, further details are provided in the following two paragraphs.

\footnotetext{
${ }^{10}$ In the derivation of the following result, the $\arctan 2(\cdot)$ operator is assumed in place of the simpler $\arctan (\cdot)$.
} 


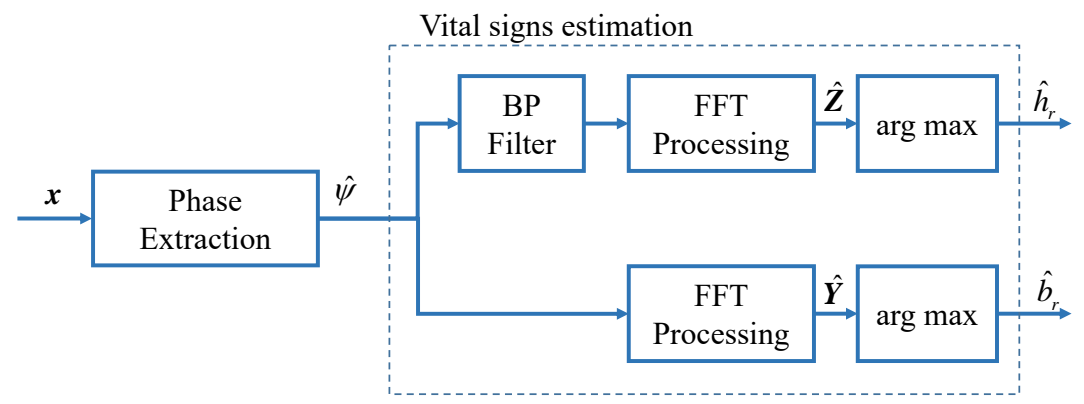

Figure 8: Representation of the signal processing chain characterising various deterministic methods for vital sign estimation.

\section{1) Frequency modulated and stepped frequency continuous wave radars}

Let us concentrate first on the simplest method that can be adopted in an FMCW radar system for the extraction of the above mentioned phase information. This method processes the received signal samples acquired over a single transmission frame (made of $N_{c}$ consecutive chirps; see Paragraph IV.B.) and consists of the following steps:

1. The $N_{0}^{\prime} \times N_{c}$ complex matrix $\mathbf{X}=[X[l, n]]$ is computed; here,

$$
X[l, n] \triangleq \frac{1}{N} \sum_{k=0}^{N-1} x[k, n] \exp \left(-j 2 \pi n l / N_{0}^{\prime}\right)
$$

with $l=0,1, \ldots, N_{0}^{\prime}-1$ and $n=0,1, \ldots, N_{c}-1$. Note that $X[l, n]$ represents the $l$-th coefficient of an $N_{0}^{\prime}$ order DFT of the signal samples acquired over the $n$-th chirp of the considered transmission frame.

2. The index of the frequency bin

$$
\hat{l} \triangleq \arg \max _{\tilde{l} \in\left\{0,1, \ldots, N_{0}^{\prime}-1\right\}}|X[\tilde{l}, n]|,
$$

associated with the target (i.e., with the chest of the subject under test) is identified.

3. Phase extraction is accomplished through the AD method (followed by phase unwrapping), but does not involve the time domain samples of the received signal (i.e., the samples $\{x[k, n]\}$ ). In fact, the samples $x_{I}[n]$ and $x_{Q}[n]$ are replaced by $\Re\{X[\hat{l}, n]\}$ and $\Im\{X[\hat{l}, n]\}$, respectively, in (56)-(58) (here, $\Re\{x\}$ and $\Im\{x\}$ denote the real part and the imaginary part of $x$, respectively).

It is important to point out that:

a) The procedure described above generates, as a by product, an estimate of the range of the target, i.e. of the distance between the radar and the chest wall of the subject under test. In fact, given $\hat{l}(61)$, such an an estimate is given by $\hat{R}_{0}=\hat{l} K_{m}$, where

$$
K_{m} \triangleq \frac{c}{2 \mu N_{0}^{\prime} T_{s}}
$$

is the bin-to-meters conversion factor.

b) Some methods, similar to the ones described above for CW Doppler radars, are available for the compensation of a DC offset or of large movements; in fact, they are based on filtering techniques or on polynomial fitting $[17,47,51]$.

c) If the distance between the chest wall and the radar is known with a certain accuracy (e.g., when the radar is employed for monitoring the vital signs of a patient in a bed), a procedure for reducing the region of interest (ROI) can be implemented to simplify the estimation of $\hat{l}$. In particular, $\hat{l}$ can be searched in the ordered set $\left\{l_{m}, l_{m}+1, \ldots, l_{M}\right\}$, made of $l_{M}-l_{m}+1$ elements; here, $l_{m}=R_{m} / K_{m}$, $l_{M}=R_{M} / K_{m}$ and $R_{m}\left(R_{M}\right)$ is the minimum (maximum) expected range. 


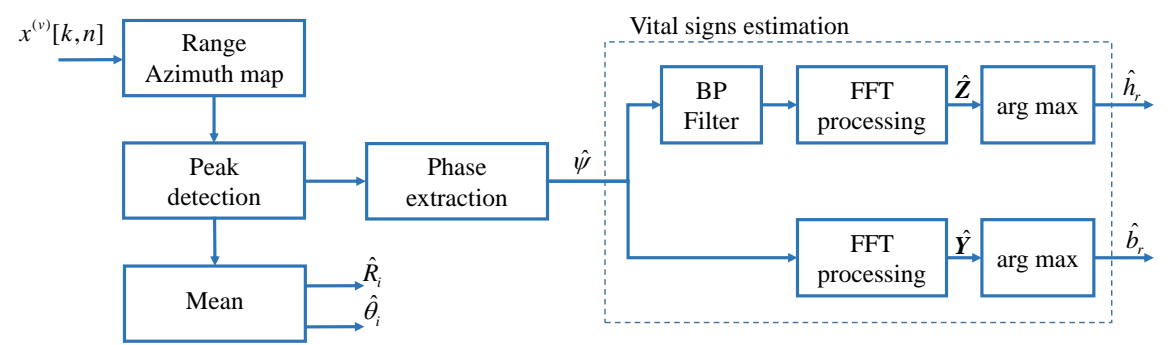

Figure 9: Representation of the signal processing described in Paragraph V.B. and employed for estimating the position (range and azimuth) and the vital signs (HR and BR) of multiple people by means of a colocated FMCW MIMO radar.

The method illustrated above for an FMCW radar system can be also employed for an SFCW radar system because of the duality relating such systems (see Paragraph 1)). The only difference is represented by the fact the definition (60) is replaced by

$$
X[l, n] \triangleq \frac{1}{N} \sum_{k=0}^{N-1} x[k, n] \exp \left(j 2 \pi n l / N_{0}^{\prime}\right),
$$

with $l=0,1, \ldots, N_{0}^{\prime}-1$ and $n=0,1, \ldots, N_{c}-1$. This means that an $N_{0}^{\prime}$ DFT is replaced by an inverse DFT (IDFT) of the same order, which is efficiently evaluated through an inverse FFT (IFFT).

\section{2) Impulse radio ultra-wideband radars}

The AD method described in Par. V.A. can be also employed for phase extraction in IR-UWB radar systems. In this case, however, the samples $x_{I}[n]$ and $x_{Q}[n]$ appearing in the RHS of (56) are replaced by $x_{I}[\hat{k}, n]$ and $x_{Q}[\hat{k}, n]$ (see (28) and (29)), respectively, with $n=0,1, \ldots, N_{c}-1$; here, $\hat{k}$ denotes the value of the fast time index $k$ corresponding to the main peak detected at the MF response ${ }^{11}$. Note also that phase estimation is followed by phase unwrapping and that, given $\hat{k}$, an estimate $\hat{\tau}$ of the detected target can easily computed; this allows to evaluate the estimate $\hat{R}_{0}=\hat{\tau} c / 2$ of the distance $R_{0}$ between the radar and the chest of subject under test (see (12)).

Finally, it is worth mentioning that:

a) The clutter contribution to the samples $\{x[k, n]\}$ can be removed by applying this sequence to an highpass filter. However, if the clutter produces small fluctuations along the slow-time axis, algorithms based on polynomial fitting can be employed for its removal [51].

b) The SNR of the received signal can be also improved by resorting to noise reduction techniques. An example of such techniques is provided in [52, Sect. 3.2], where the empirical mode decomposition (EMD) is exploited for noise mitigation. In this case, the sequence $\{x[k, n]\}$ is decomposed into a superposition of intrinsic sub-signals, defined at precise instantaneous frequencies and called intrinsic mode functions (IMFs). This method allows to retain only the most important frequency components of the input signal and filter out the oscillations associated with noise components.

Some alternatives to the FFT-based estimation method described in Paragraph V.A. are also available in the technical literature. These are based on the evaluation of a fourth order cumulant [53] or of a continuous wavelet transform (CWT); note that the use of the CWT in place of the FFT allows to analyse how the frequency components of the received signal phase evolve over time [52].

\section{V.B. Estimation of vital signs of multiple subjects through multiple-input multiple- output radars}

In the last few years, increasing attention is being paid to MIMO radar systems, mainly because they make range and DOA estimation of multiple targets possible. The use of MIMO FMCW, IR-UWB and CW radar

\footnotetext{
${ }^{11}$ It is assumed that $\hat{k}$ does not change in the considered frame interval for simplicity.
} 
systems for the monitoring of vital signs has been investigated in [54-58], [59,60] and [61] respectively. More specifically, the use of MIMO FMCW radars operating at $77 \mathrm{GHz}$ and at $120 \mathrm{GHz}$ has been studied in [54-56] and [58], respectively, whereas the simultaneous use of two FMCW radars, each endowed with single ULA, but one operating at $24 \mathrm{GHz}$ and the other one at $77 \mathrm{GHz}$, has been investigated in [57]. Reference [61], instead, focus on the use of beamforming in MIMO CW radar systems.

Let us illustrate now some key concepts that are useful to understand the processing accomplished at the RX side of a MIMO radar system. To begin, we focus on a FMCW MIMO radar equipped with $N_{T}$ TX and $N_{R}$ RX antennas (and, consequently, with $N_{V}=N_{T} N_{R}$ VAs; see (33)). Under the assumptions illustrated in Paragraph IV.C., the signal samples acquired in a single frame through the $N_{V}$ VAs can be collected in $N \times N_{V} \times N_{c}$ matrix $\mathbf{x} \triangleq\left\{x^{(v)}[k, n]\right\}$, where

$$
x^{(v)}[k, n] \triangleq \sum_{i=0}^{L-1} A_{i, n}^{(v)} \exp \left(j 2 \pi k F_{i, n}^{(v)}\right)+w^{(v)}[k, n],
$$

with $k=0,1, \ldots, N-1, n=0,1, \ldots, N_{c}-1$ and $v=0,1, \ldots, N_{V}-1$; here, $L$ is the overall number of targets, and $A_{i, n}^{(v)}\left(F_{i, n}^{(v)}=f_{i, n}^{(v)} T_{s}\right)$ is the complex amplitude (the normalised version of the frequency $f_{i, n}^{(v)}$ ) characterising the $i$-th target observed on the $v$-th VA in the $n$-th chirp interval. A processing method that can be adopted in this case to estimate the range, the DOA and the vital signs of multiple people in the considered radar system is described by the block diagram represented in Fig. 9. Such a method consists in the four steps listed below.

1. Computation of the range-azimuth map - A size $N_{0}^{\prime} \times N_{A}$ 2D-FFT of the $N \times N_{V}$ input matrix $\mathbf{x}[n]=\left[x^{(v)}[k, n]\right]$ (with $v=0,1, \ldots, N_{V}-1$ and $\left.k=0,1, \ldots, N-1\right)$ is computed in the $n$-th chirp interval (with $n=0,1, \ldots, N_{c}-1$ ); this produces the $N_{0}^{\prime} \times N_{A}$ matrix

$$
\mathbf{X}_{n}=\left[X^{(n)}[l, m]\right]
$$

with $l=0,1, \ldots, N_{0}^{\prime}-1$ (see Paragraph 1)) and $m=-N_{A} / 2,-N_{A} / 2+1, \ldots, N_{A} / 2-1$. Then, the $n$-th range-azimuth map is evaluated as

$$
\mathbf{J}[n] \triangleq\left[\left|X^{(n)}[l, m]\right|\right]
$$

with $n=0,1, \ldots, N_{c}-1$.

2. Peak detection - The peaks appearing in the $n$-th range-azimuth map $\mathbf{J}[n]$ are detected by means of a proper method (for instance, the so called constant false alarm rate, CFAR, method can be adopted [62]), since each of them reveals the presence of a potential target. Let us assume that, independently of $n, \hat{L}$ peaks are detected in the azimuth map and that the position of the $i$-th peak (with $i=0,1, \ldots, \hat{L}-1)$ in the $n$-th map $\mathbf{J}[n]$ is identified by the couple $\left(\hat{l}_{i}[n], \hat{m}_{i}[n]\right)\left(\right.$ with $\left.n=0,1, \ldots, N_{c}-1\right)$. Then, estimates of the range and of the azimuth of the target associated with the $i$-th peak can be evaluated as

$$
\hat{R}_{i}[n]=K_{m} \hat{l}_{i}[n]
$$

and

$$
\hat{\theta}_{i}[n]=\arcsin \left(\hat{m}_{i}[n] f_{r}\right),
$$

respectively; here, $K_{m}$ is expressed by (62) and $f_{r}=2 / N_{A}$ (see eqs. (19)-(20) and (51)). Given the set $\left\{\left(\hat{l}_{i}[n], \hat{m}_{i}[n]\right) ; i=0,1, \ldots, \hat{L}-1\right\}$, the $\hat{L} \times N_{c}$ matrix

$$
\overline{\mathbf{X}}=[\bar{X}[i, n]]
$$

is generated; here,

$$
\bar{X}[i, n]=X^{(n)}\left[\hat{l}_{i}[n], \hat{m}_{i}[n]\right]
$$

with $i=0,1, \ldots, \hat{L}-1$ and $n=0,1, . ., N_{c}-1$. 
3. Arctangent demodulation and phase unwrapping - In this step, the $i$-th row of the matrix $\overline{\mathbf{X}}(69)$ undergoes $\mathrm{AD}$ followed by phase unwrapping (with $i=0,1, \ldots, \hat{L}-1$ ); this produces the phase vector

$$
\hat{\boldsymbol{\psi}}_{i} \triangleq\left[\psi_{i}[0], \psi_{i}[1], \ldots, \psi_{i}\left[N_{c}-1\right]\right]^{T} .
$$

4. Breath and heart rate estimation - The phase vector $\hat{\psi}_{i}$ undergoes FFT processing (with $i=0,1, \ldots, \hat{L}-$ 1 ); this produces the $N_{m}$-dimensional spectrum $\overline{\boldsymbol{Y}}_{i}$ (whose $\tilde{b}$-th element is expressed by (49)). Then, an estimate $\hat{b}_{r}[i]\left(\hat{h}_{r}[i]\right)$ of the BR (HR) associated with the $i$-th target is evaluated on the basis of (48) $((54))$.

At the end of the procedure described above, the range estimate

$$
\hat{R}_{i}=\frac{1}{N_{c}} \sum_{n=0}^{N_{c}-1} \hat{R}_{i}[n]
$$

and the azimuth estimate

$$
\hat{\theta}_{i}=\frac{1}{N_{c}} \sum_{n=0}^{N_{c}-1} \hat{\theta}_{i}[n]
$$

can be computed for the $i$-th target by averaging the corresponding estimates evaluated over each of the $N_{c}$ chirps forming the considered transmission frame (with $i=0,1, \ldots, \hat{L}-1$ ).

Finally, it is important to point out that the procedure illustrated above for a MIMO FMCW radar system can be easily adapted to MIMO IR-UWB and SFCW radars; in the last case, FFTs must be replaced by IFFTs.

\section{V.C. Numerical results}

In this paragraph, we show some numerical results generated by applying various estimation methods illustrated above to a set of synthetically generated data. We first focus on a SISO FMCW radar and a SISO SFCW radar, both placed in front of the chest of a single subject at the distance $d=0.5 \mathrm{~m}$. The following assumptions have been made in generating our dataset:

a) The model described by (1)-(4) is adopted for the chest displacement of the subject (modelled as a single point target for simplicity); its parameters are the same as those listed in Par. III.B.).

b) The FMCW and SFCW radars are characterised by the following parameters: a) carrier frequency $f_{0}=77 \mathrm{GHz}$ (the corresponding wavelenght is $\lambda=c / f_{0}=3.9 \mathrm{~mm}$ ); b) bandwidth $B=2 \mathrm{GHz} ; \mathrm{c}$ ) number of samples $N=256$ (in the time domain for each chirp of the FMCW radar, in the frequency domain for each frequency modulated pulse of the SFCW radar); d) chirp (pulse) repetition period $T_{0}=0.06 \mathrm{~s}$ for the FMCW (SFCW) radar; e) number of chirp/frame (pulses/frame) $N_{c}=1034$ for the FMCW (SFCW) radar; f) ADC sampling frequency $f_{s}=9 \mathrm{MHz}\left(f_{s}=7.8 \mathrm{MHz}\right)$ for the FMCW (SFCW) radar. Moreover, the ramp parameters of the FMCW are: a) reset time $T_{R}=0$; b) ramp time $T=N / f_{s}=28 \mu \mathrm{s} ;$ c) chirp slope $\mu=70.312 \mathrm{MHz} / \mu \mathrm{s}$.

c) In both systems the frame duration is $T_{F}=N_{c} T_{0} \cong 60 \mathrm{~s}$; this parameter represents also the duration of our observation interval.

d) The real and imaginary part of the AWGN noise samples $\{w[k, n]\}$ appearing in the RHS of (21) and (25) have zero mean and variance $\sigma_{n}^{2}=0.1$.

Our synthetically generated data have been processed by means of the deterministic method described in Par. V.A. and its overall architecture is represented in Fig. 8. The AD method followed by phase unwrapping has been used for phase estimation and the following choices have been made: a) DFT orders $N_{0}^{\prime}=512$ and $N_{0}=\bar{N}_{0}=M N_{c}=4 \cdot 1034$; b) $K_{m}=31$ (bin-to-meters conversion factor; see (62)); c) the bandpass filter employed in HR estimation is a fourth-order Butterworth filter and the lower (upper) limit of its passband is $f_{L}=50 / 60 \cong 0.83 \mathrm{~Hz}\left(f_{U}=100 / 60 \cong 1.7 \mathrm{~Hz}\right)$, so that its bandwidth is $B_{B P}=f_{U}-f_{L} \cong 0.83 \mathrm{~Hz}$. 


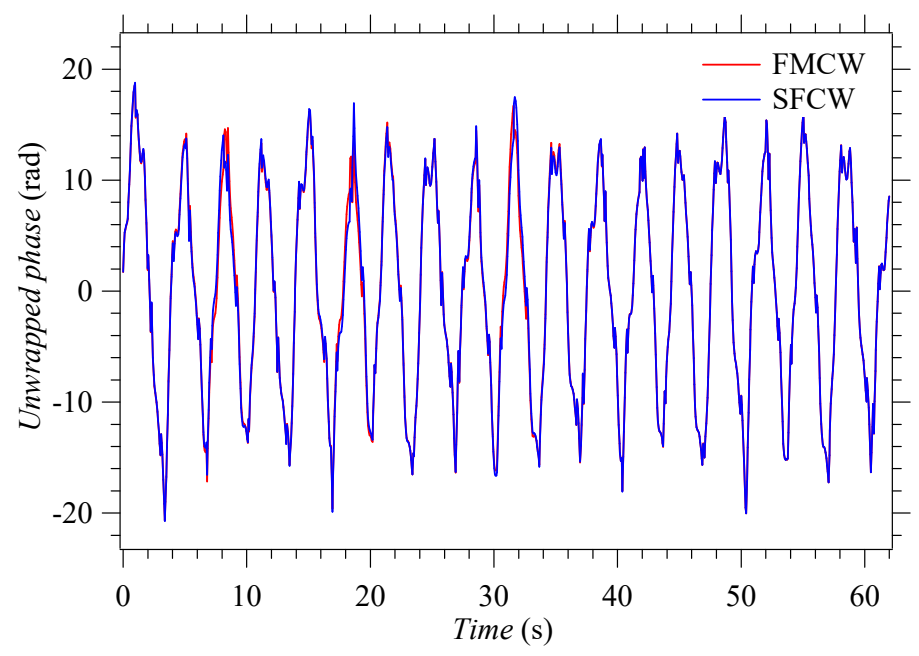

Figure 10: Representation of the elements of the phase vector $\hat{\boldsymbol{\psi}}$ (46) resulting from synthetically generated measurements. Both FMCW and SFCW radar systems are considered.

The elements of the phase vector $\hat{\boldsymbol{\psi}}$ (46) obtained for the FMCW and the SFCW radar systems are represented in Fig. 10. The amplitude spectrum obtained at the output of the slow-time FFT (IFFT) executed for phase estimation in the FMCW (SFCW) radar system is shown in Fig. 11, whereas the amplitude spectrum evaluated after BPF of the phase vector is shown in Fig. 12. The results obtained for the considered radar systems are similar and deserve the following comments:

a) The phase signal does provide information about the dynamics of chest displacement; in fact, comparing Fig. 10 with Fig. 1 leads to the conclusion that its evolution is similar to that characterising the chest displacement in the considered scenario.

b) The peaks appearing in the amplitude spectrum shown in Fig. 11 are associated not only with the BR and the HR, but also with the harmonics of the BR. Note, in particular, that the third harmonic falls inside the passband of the filter and that its presence may lead to a wrong estimate of the HR.

c) Based on the available spectra, the estimates $\hat{b}_{r}=17.9 \mathrm{acts} / \mathrm{min}$ and $\hat{h}_{r}=58.8 \mathrm{acts} / \mathrm{min}$ evaluated for the $\mathrm{BR}$ and the HR, respectively are very accurate since the exact values of these rates are $b_{r}=18$ acts/min and $h_{r}=60$ acts/min, respectively.

Let us focus now on a MIMO FMCW radar system placed in front of the chest of three static subjects (the $i$-th subject is denoted $\mathrm{P}_{i}$, with $i=1,2$ and 3 ), whose range $(R)$, azimuth $(\theta)$, $\operatorname{HR}\left(h_{r}\right)$ and $\mathrm{BR}\left(b_{r}\right)$ are listed in Table 4 . In this case, the following assumptions have been made in synthetically generating our dataset:

a) The chest displacement characterising each person is modelled by (1)-(4); moreover, the values of all the model parameters are the same as those employed in paragraphs III.B. and V.A..

b) The values of the parameters $f_{0}, B, N, T_{0}, N_{c}, f_{s}, T_{R}, \mu$ of the employed MIMO radar are the same as those listed for the SISO FMCW radar considered above. Moreover, the MIMO radar is endowed with a virtual ULA like the one shown in Fig. 7-b); the overall number of VAs forming this array is $N_{V}=16$, wheras the distance between adjacent virtual elements is $d=\lambda / 4$.

Our data have been processed by the deterministic algorithm illustrated in Paragraph V.B.. The rangeazimuth map $\mathbf{J}[0]$ generated on the basis of the measurements acquired through the MIMO radar in the first chirp interval is represented in Fig. 13. The peaks detected by the CFAR algorithm are indicated by small circles, whereas black crosses are used to identify the centroids ${ }^{12}$ of each cluster of adjacent peaks.

\footnotetext{
${ }^{12}$ Note that each centroid represents the position estimated for one of the subjects.
} 


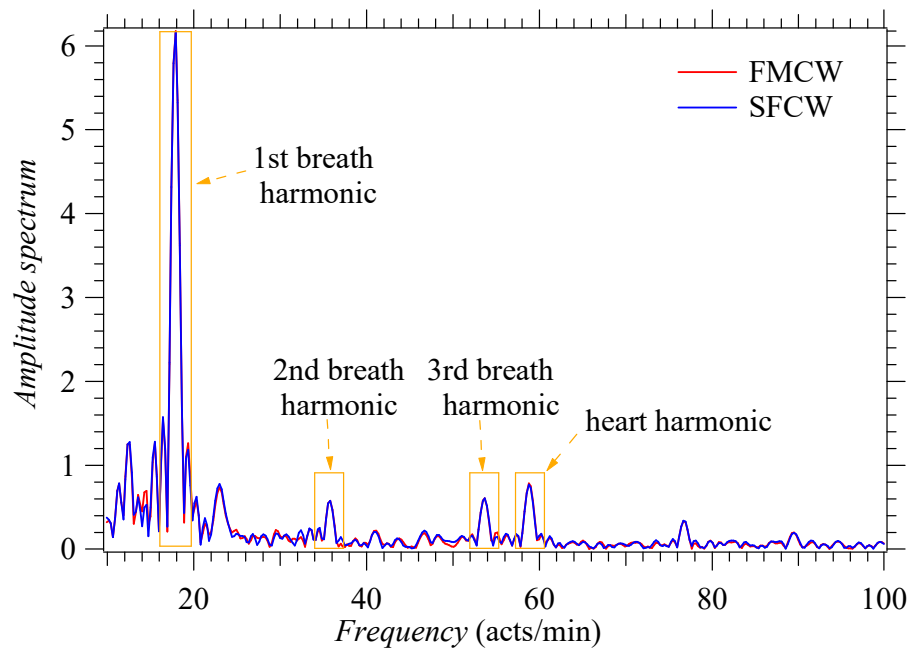

Figure 11: Representation of the amplitude spectrum referring to the the phase vector $\hat{\psi}$ (46). Both FMCW and SFCW radar systems are considered.

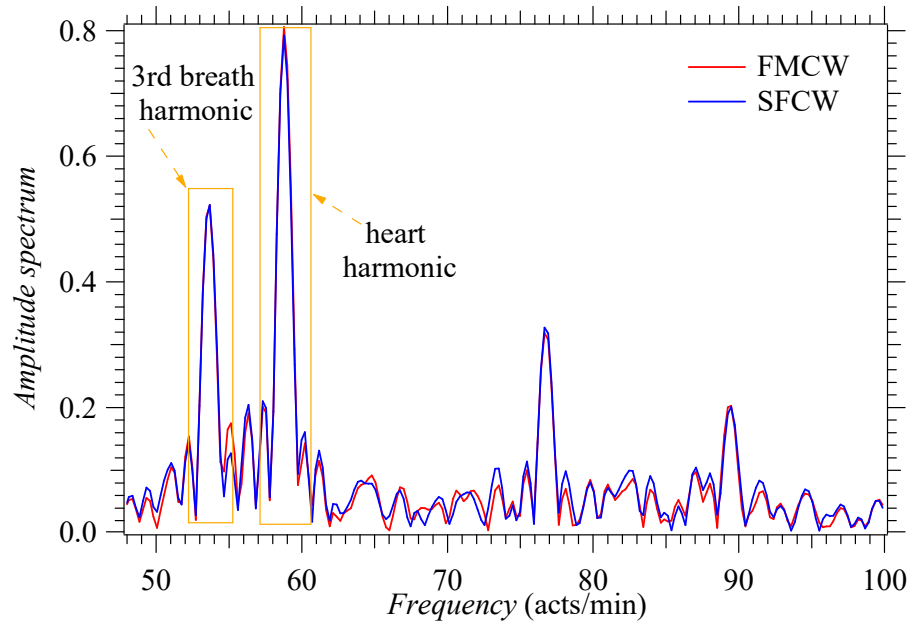

Figure 12: Representation of the amplitude spectrum of the sequence generated through bandpass filtering of the phase vector $\hat{\boldsymbol{\psi}}$ (46). Both FMCW and SFCW radar systems are considered. 


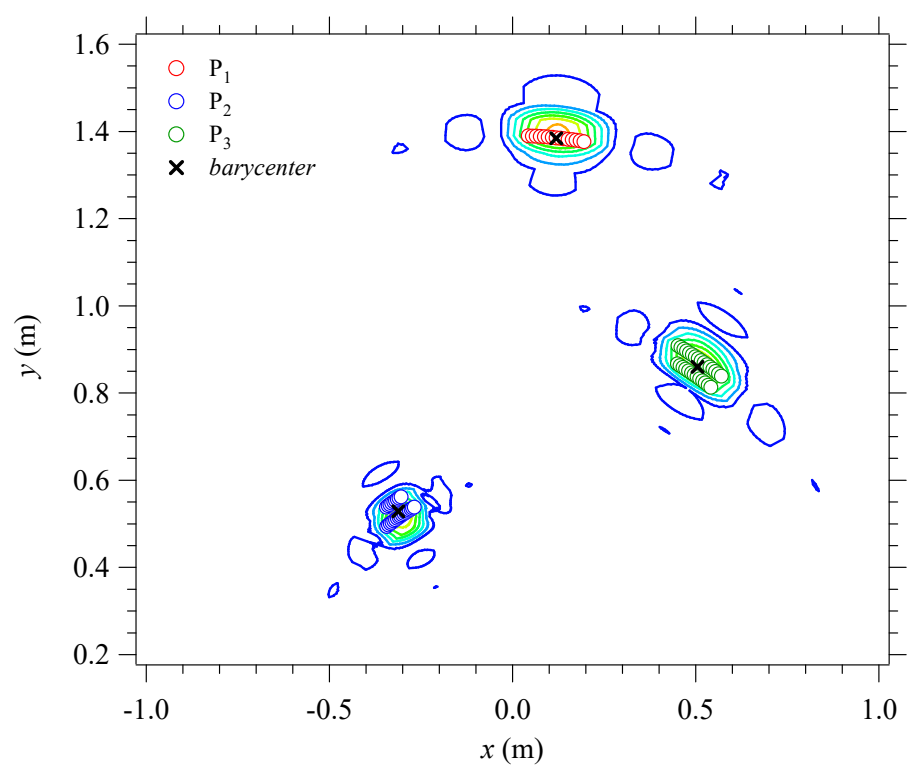

Figure 13: Range-azimuth map generated on the basis of our synthetically generated data referring to a MIMO radar system. The peaks detected by the CFAR algorithm are identified by small circles, whereas black crosses indicate the centroid of each cluster of peaks.

Table 4: Exact values and corresponding estimates of the following parameters (referring to three distinct subjects): range $(R)$, azimuth $(\theta)$, breath rate $\left(b_{r}\right)$ and heart rate $\left(h_{r}\right)$.

\begin{tabular}{ccccccccc}
\hline Person & $\begin{array}{c}R \\
(\mathrm{~m})\end{array}$ & $\begin{array}{c}\hat{R} \\
\left({ }^{\circ}\right)\end{array}$ & $\begin{array}{c}\hat{\theta} \\
\text { acts/min }\end{array}$ & $\begin{array}{c}\hat{b}_{r} \\
\text { acts/min }\end{array}$ \\
\hline $\mathrm{P}_{1}$ & 0.60 & 0.61 & -30 & -30.5 & 14.0 & 13.8 & 70 & 66.8 \\
\hline $\mathrm{P}_{2}$ & 1.00 & 1.01 & 30 & 30.3 & 17 & 16.9 & 66.0 & 63.1 \\
\hline $\mathrm{P}_{3}$ & 1.40 & 1.39 & 5 & 4.9 & 20.0 & 19.8 & 57.0 & 55.6 \\
\hline
\end{tabular}

The elements of the phase vector $\hat{\boldsymbol{\psi}}(46)$ and of its amplitude spectrum in correspondence of the centroid obtained for the first (closest to the radar) target are shown in Fig. 14-a) and 14-b), respectively, whereas the estimates of the positions of the three subjects together with the estimates of their BR and HR are listed in Table 4. From these results the following conclusions can be easily inferred:

a) The range-azimuth map allows to detect all the subjects and estimate their position.

b) The phase vector associated with each centroid provides important information about the dynamic of the chest displacement of the subject associated with it.

c) The position and vital signs estimated for each subject are reasonably accurate.

\section{V.D. Detection and estimation algorithms based on artificial intelligence}

Various deterministic algorithms may fail in complex, highly dynamic and time varying scenarios [41]. In such cases, learning methods, based on artificial intelligence (AI), may be extremely useful, since they are able to: a) learn the regularities characterizing the raw data acquired by radar systems; b) automatically extract information from them. These methods are divided in machine learning (ML) and deep learning (DL) techniques [63]. On the one hand, the former class of techniques exploits a customized set of features, 


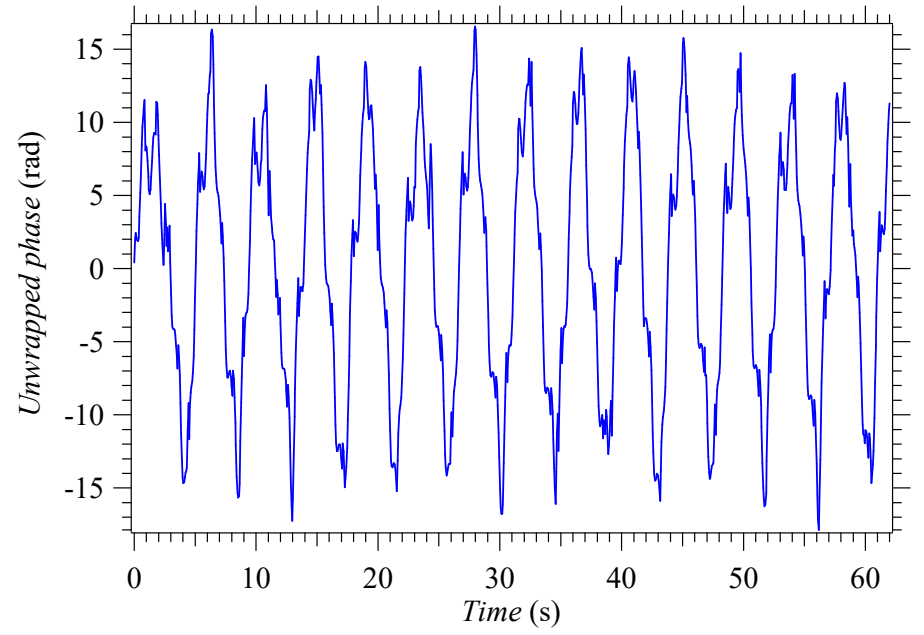

(a)

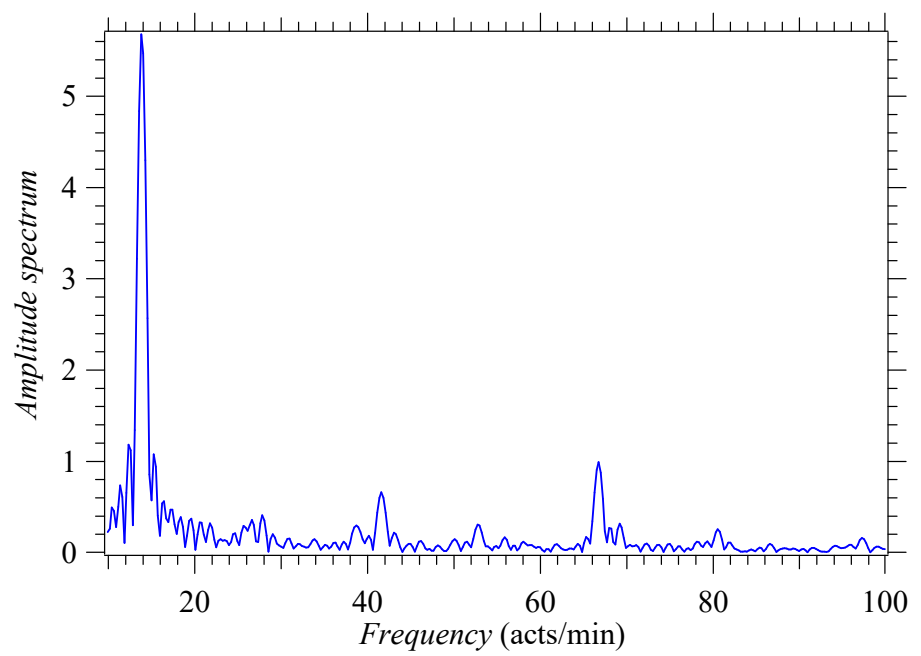

(b)

Figure 14: Representation of: a) the elements of a phase vector generated on the basis of the measurements acquired through a MIMO FMCW radar during a single chirp interval; b) its amplitude spectrum. The target closest to the radar is considered. 


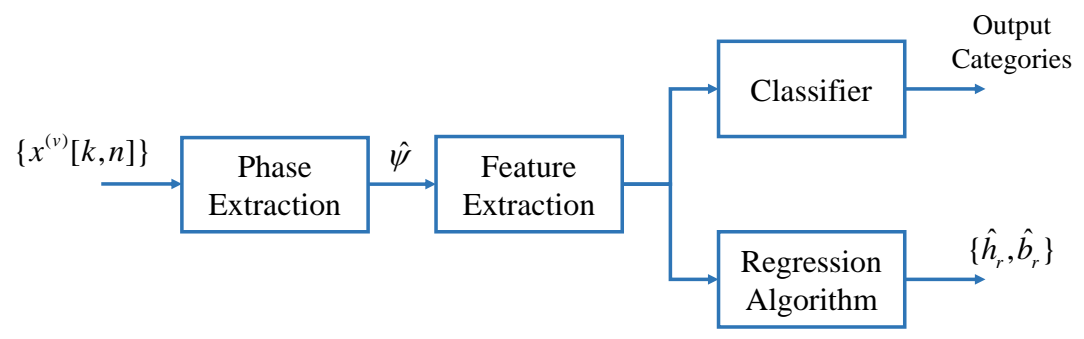

Figure 15: Block diagram representing the signal processing chain of a radar-based system employing a learning method for vital sign monitoring.

manually extracted from the available raw data by means of different processing techniques. On the other hand, the latter class can learn specific data patterns and extract useful information directly from the same data through the use of neural networks; this approach requires limited expertise on signal analysis in radar systems. Each class of methods includes supervised and unsupervised learning technique. Generally speaking, supervised techniques, mainly used for solving classification or regression problems, are based on the idea of exploiting a labelled dataset for learning from them through a specific procedure, called training. Training aims at the identification of the model, i.e. at estimating of the probability density function (also called predictive distribution) on the basis of which the available dataset has been generated. Given the model learnt during training (i.e., the predictor), the label associated with a completely new observation or the value of a continuous variable related to it can be predicted in a way that a specific loss (known as generalisation loss) is minimised; such tasks are known as classification and regression, respectively. Well known ML methods for classification are the K-nearest neighbours (K-NN), the support vector machine (SVM) and the ensemble classifier [64].

Unsupervised methods, instead, do not require a labelled dataset and deal with learning some specific properties of the mechanism on which the generation of the considered dataset is based. They can be exploited to solve specific technical problems, like data clustering, dimensionality reduction and feature selection. In practice, data clustering aims at partitioning the available dataset in a number of groups such that data points in the same group are dissimilar from the data points belonging to all the other groups. Dimensionality reduction is employed to generate a reduced dimensionality representation of the observations, whereas feature selection consists in deriving a vector valued function that produces a useful and lower-dimensional representation of the available feature.

In general, the dataset employed to train a specific method contains $N_{t}$ couples, each of which consists of a $D_{r}$-dimensional real vector of features and a $D_{t}$-dimensional real vector of labels. The size $N_{t}$ of the dataset can be reasonably small in the case of ML (say, between a few dozen and a few hundred), but is substantially larger in the case of DL (say, at least one thousand). In the case of radar-based monitoring of vital signs, a vector of customized features can be obtained from the phase vector $\hat{\boldsymbol{\psi}}(46)$ through a procedure called feature extraction and can be processed for classification purposes. For instance, different breathing diseases could be recognised on the basis of several features, such as the breathing frequency, the chest displacement and the variability of the breathing frequency in short and long observation time. Generally speaking, for all the radar topologies, the extracted features can be grouped in three different classes, namely time, short-term and time-frequency domain features. Time features are represented by various characteristics of the evolution of the elements of the phase vector $\hat{\boldsymbol{\psi}}$, such as their average (or maximum) peak amplitude, the variability of their amplitude and the number of peaks. Short-time features (time-frequency domain features ${ }^{13}$ ), instead, allow to monitor how the energy (the spectral content) of the elements of the phase vector $\hat{\boldsymbol{\psi}}$ evolves. The above mentioned features can be employed for both classification and regression, as exemplified by Fig. 15, where the overall signal processing chain of a radar system employing a ML method is illustrated.

An alternative to ML methods is provided by DL methods. In the last case, feature extraction is automatically accomplished by a neural network and, if classification is required, a softmax layer is employed to evaluate the probability that a certain observation is associated with one of the given classes.

A limited literature on the use of ML and DL methods in radar-based monitoring of vital signs is available.

\footnotetext{
${ }^{13}$ The CWT can be employed to extract these features.
} 
The use of ML methods in CW Doppler radar systems for vital sign monitoring has been investigated in [65-68], while an unsupervised method for DC offset and clutter suppression using FMCW radars has been investigated in [69]. Moreover, an HR estimation technique based on a convolutional neural network (CNN) has been developed in [70] for an IR-UWB radar system. In particular, supervised methods have been exploited for classifying breathing disorders, for heart sound classification and for removing the high order harmonics from the phase vector $\boldsymbol{\psi}(46)$ in [66], [68] and [65], respectively. An artificial neural network (ANN) for reliable detection of heartbeats through a CW Doppler radar has been proposed in [67]. The adopted ANN is composed by a cascade of multiple layers with different number of neurons and its architecture is quite simple; its main drawback is represented by its inability to cope with time series, i.e. to extract features related to the time evolution of the observed signal.

\section{Applications of the Radar Technology to Vital Sign Monitoring}

In this section, we propose a synopsis of the technical literature concerning the following specific issues: a) the monitoring of $\mathrm{HR}$ and $\mathrm{BR}$; b) the experimental setups adopted in vital sign montoring; $\mathrm{c}$ ) the monitoring of heart sounds.

\section{VI.A. Heart rate and breath rate monitoring}

The use of radar systems for monitoring HR and BR has been investigated by several research groups, whose work has allowed to assess the performance achieved by different radar prototypes operating at distinct frequencies and radiating heterogeneous signals. As far as the use of the radar technologies described in Paragraph IV.A. is concerned, it is worth mentioning that:

a) Continuous wave Doppler radars operating at $2.4 \mathrm{GHz}$, at $5.8 \mathrm{GHz}$ and at $24 \mathrm{GHz}$ have been employed to measure respiration, heartbeat, or motion activity in $[3,12,71,72]$, in [73] and in $[20,67,74]$, respectively.

b) Frequency modulated continuous wave radars operating in the $\mathrm{C}$ and $\mathrm{X}$ bands, at $24 \mathrm{GHz}$, at $77 \mathrm{GHz}$, and at $122 \mathrm{GHz}$ have been used to simultaneously estimate of the the vital parameters of multiple people in [22,75], in [76,77], in [31,51] and in [78], respectively.

c) Stepped frequency continuous wave radars operating at frequencies lower than $3 \mathrm{GHz}$ and in the $\mathrm{X}$ band are have been exploited for the estimation of the vital signs parameters of single or multiple people in $[79,80]$ and in $[81,82]$, respectively.

d) Impulse radio UWB radars operating in the $\mathrm{C}, \mathrm{X}$ and $\mathrm{E}$ bands and having large bandwidths $(2-3$ $\mathrm{GHz})$ have been employed to measure vital parameters with high accuracy in [21,52,70,83-85].

Model-based (MB) or learning-based (LB) methods have been employed for the processing of the measurements provided by the above mentioned radar systems; moreover, such measurements have been acquired over population of different sizes. Essential information about the adopted processing methods and the size of the involved population are summarised in Table 5; note that, in this table, the size $N_{p}$ of the population (low, L, medium, $\mathrm{M}$, or high, $\mathrm{H})$ on which it has been tested have been specified ${ }^{14}$. Some details about the most important processing methods and the main results achieved through their use are provided below.

Continuous wave Doppler radars - Three novel deterministic techniques for estimating vital signs have been developed in [71], where a radar system operating at $2.4 \mathrm{GHz}$ has been tested on a (single) human subject located at a fixed distance. These techniques are based on: a) the FFT processing of the timedomain phase signal estimated through $\mathrm{AD}$ (see the block diagram represented in Fig. 8 and in Par. V.A.); b) the computation of the autocorrelation of the phase vector $\hat{\psi}(46)$ to estimate the period of the timedomain phase signal; c) the FFT processing of the above mentioned autocorrelation. The last two techniques are shown to achieve a better accuracy than the first one. Other interesting results are offered by [3], in

\footnotetext{
${ }^{14}$ In this table, the population size is low, medium or high if $1<N_{p} \leq 10,10<N_{p} \leq 20$ or $N_{p}>20$, respectively.
} 
Table 5: Classification of the references cited in Paragraphs VI.A. and VI.B. on the basis of: a) the size of thw acquired dataset; b) the position of the subject/subjects under test; c) the employed radar technology; d) the category of the adopted signal processing method.

\begin{tabular}{|c|c|c|c|c|c|c|c|c|c|c|c|}
\hline \multirow{2}{*}{ Work no. } & \multirow{2}{*}{$\begin{array}{l}\text { Dataset } \\
\text { size }\end{array}$} & \multicolumn{3}{|c|}{ Position of the involved subjects } & \multicolumn{4}{|c|}{ Radar technology } & \multirow{2}{*}{$\begin{array}{c}\text { Freq. } \\
\text { GHz }\end{array}$} & \multicolumn{2}{|c|}{ Signal proc } \\
\hline & & sitting & $\begin{array}{l}\text { lying } \\
\text { down }\end{array}$ & other & CW & FMCW & SFCW & UWB & & MB & LB \\
\hline [71] & $\mathrm{H}$ & $\checkmark$ & & & $\checkmark$ & & & & 2.4 & $\checkmark$ & \\
\hline [73] & $\mathrm{H}$ & $\checkmark$ & & & $\checkmark$ & & & & 5.8 & $\checkmark$ & \\
\hline [3] & $\mathrm{L}$ & & & $\checkmark$ & $\checkmark$ & & & & 2.4 & $\checkmark$ & \\
\hline [72] & M & & $\checkmark$ & & $\checkmark$ & & & & 2.4 & & $\checkmark$ \\
\hline [67] & M & $\checkmark$ & & & $\checkmark$ & & & & 24 & & $\checkmark$ \\
\hline$[74]$ & M & & $\checkmark$ & & $\checkmark$ & & & & 24 & $\checkmark$ & \\
\hline$[20]$ & $\mathrm{L}$ & & $\checkmark$ & & $\checkmark$ & & & & 24 & $\checkmark$ & \\
\hline$[12]$ & $\mathrm{L}$ & & & $\checkmark$ & $\checkmark$ & & & & 2.4 & $\checkmark$ & \\
\hline$[76]$ & $\mathrm{L}$ & & $\checkmark$ & & & $\checkmark$ & & & 24 & $\checkmark$ & \\
\hline$[78]$ & $\mathrm{L}$ & $\checkmark$ & $\checkmark$ & & & $\checkmark$ & & & 122 & $\checkmark$ & \\
\hline [51] & $\mathrm{L}$ & & $\checkmark$ & & & $\checkmark$ & & & 77 & $\checkmark$ & \\
\hline$[22]$ & $\mathrm{L}$ & $\checkmark$ & & & & $\checkmark$ & & & 5.8 & $\checkmark$ & \\
\hline [77] & M & $\checkmark$ & & & & $\checkmark$ & & & 24 & $\checkmark$ & \\
\hline$[75]$ & $\mathrm{L}$ & & & $\checkmark$ & & $\checkmark$ & & & 10 & $\checkmark$ & \\
\hline [79] & $\mathrm{M}$ & & $\checkmark$ & & & & $\checkmark$ & & $0.3-1.3$ & $\checkmark$ & \\
\hline$[80]$ & $\mathrm{M}$ & $\checkmark$ & & & & & $\checkmark$ & & 2.4 & $\checkmark$ & \\
\hline [81] & $\mathrm{L}$ & & & & & & $\checkmark$ & & 9.0 & $\checkmark$ & \\
\hline$[82]$ & $\mathrm{L}$ & & & & & & $\checkmark$ & & 5.8 & $\checkmark$ & \\
\hline$[31]$ & $\mathrm{L}$ & $\checkmark$ & & & & $\checkmark$ & & $\checkmark$ & 77 & $\checkmark$ & \\
\hline$[52,83]$ & $\mathrm{L}$ & $\checkmark$ & & & & & & $\checkmark$ & 6.8 & $\checkmark$ & \\
\hline$[84]$ & $\mathrm{L}$ & $\checkmark$ & & $\checkmark$ & & & & $\checkmark$ & 4.3 & $\checkmark$ & \\
\hline$[85]$ & $\mathrm{H}$ & & $\checkmark$ & & & & & $\checkmark$ & 94 & $\checkmark$ & \\
\hline [21] & $\mathrm{H}$ & $\checkmark$ & & & & & & $\checkmark$ & 7.25 & & $\checkmark$ \\
\hline [70] & $\mathrm{H}$ & $\checkmark$ & & & & & & $\checkmark$ & 79 & & $\checkmark$ \\
\hline
\end{tabular}


which a deterministic method based on the block diagram appearing in Fig. 8 is adopted to process the measurements acquired through a mmwave Doppler radar. In that manuscript it is shown that the HR estimated on the basis of an electrocardiogram (ECG) is highly correlated with that estimated on the basis of the measurements acquired through the devised radar system.

Some potential benefits originating from the use of ML methods are illustrated in [72], where the problem of recognizing and classifying breathing disorders of various patients during their sleep is investigated. Five ML techniques, namely, SVM, linear discriminant analysis (LDA), K-NN, decision tree (DT) and ensemble learning techniques, trained on a manually selected set of features are tested; the obtained results evidence that all the considered techniques are able to achieve an high accuracy in the classification of breathing disorders.

Frequency modulated continuous wave radars - In [76] the BR and HR of a single person have been measured through an FMCW radar and an ECG; moreover, a video camera has been also used to inspect the chest dynamics of the patient under test. A dataset composed by the measurements acquired from six different people has been used for validating the adopted signal processing methods. Two different methods have been tested, one based on the FFT (similar to the one described by Fig. 8 and in Par. 1)), the other one based on the computation of the autocorrelation of the phase vector $\hat{\psi}(46)$. The obtained results have evidenced that: a) both BR and HR can be accurately extracted from radar measurements; b) the estimation of HR in the presence of the respiration harmonics can be challenging. Methods similar to the ones employed in [76] have been successfully exploited in [78] and in [77], for simultaneously estimating the vital signs of multiple people and for identifying the vital parameters of ten subjects that experience different sleep scenarios, respectively.

Stepped frequency continuous wave radars - An FFT-based estimation method, similar to the one described in Paragraph V.A., has been employed in $[79,80]$ to estimate the BR and HR of multiple subjects in a room; these subjects have been sitting on a chair or lying down in a bed, and have been characterized by different orientations. In [80], radar-based estimates have been compared with the HR and BR estimates provided by a contact reference sensor. The employed radar system has been shown to achieve the best performance when the chest of the subject under test is in front of it; however, even if this condition is not met, radar-based estimates are still sufficiently accurate. In [79], the performance of radar-based monitoring in scenarios in which obstacles, characterised by different shapes and materials (like walls), are placed between the radar and the subject under test are evaluated. Finally, an SFCW radar system has been employed in $[81,82]$ to simultaneously estimate the vital signs of multiple people. It is also worth mentioning that the estimation method devised in [82] consists in applying a CWT to the phase vector $\hat{\boldsymbol{\psi}}(46)$ in order to separate the breath contribution from the one due to heart.

Impulse radio UWB radars - An FFT-based estimator (similar to the one described by the block diagram of Fig. 8 and in Par. 2)) and the estimator based on autocorrelation of the phase vector $\hat{\boldsymbol{\psi}}$ (46) have been employed in [85] and [84], respectively, where the raw data acquired through an UWB radar system have been processed to extract the vital signs of different subjects. The accuracy achieved by an IR-UWB radar system in vital sign estimation has been compared with that of an FMCW radar system in [31]; the latter system is shown to outperform the former one thanks to its ability to perform clutter suppression. Note that, as shown in [83], noise and clutter affecting the measurement acquired through an IR-UWB radar can be mitigated by means of Kalman filtering.

\section{VI.B. Radar setup in real world scenarios}

Radar systems can be potentially exploited for remote monitoring of vital signals as conveniently and as easily as wearable devices. Readers should not forget, however, that essential requirements for their adoption in real world applications are represented by their ease of use and accuracy. Various results about the accuracy of radar systems for vital sign monitoring in the real world and often challenging scenarios can be found in $[20,22,51,75,77,86]$; note that [51], [22] and [77] concern FMCW radar systems, whereas [20,75] and [86] CW Doppler and IR-UWB radars, respectively. More specifically, the use of radar systems in an hospital for measuring the vital signs of patients has been investigated in $[20,22,51]$, whereas more challenging scenarios have been taken into consideration in $[75,77,86]$. A brief description of the experimental setup adopted in the measurement campaigns conducted by the authors of the manuscripts cited above and of the main results extracted from their experimental data is provided below. 
a) The use of a MIMO FMCW radar in an hospital bedroom has been investigated in [51]. The radar device has been positioned on the ceiling of a room, in front of the bed on which a static patient was lying down; moreover, the patient was facing up the radar during the data acquisition process, that lasted $40 \mathrm{~min}$. A good correlation between the BR and HR provided by a reference sensor and their estimates provided by the radar system has been found.

b) An FMCW radar endowed with a custom array of antennas has been employed to estimate the vital signs of a person sitting on a chair in [22]. The analysed measurements have been acquired on five subjects (three males and two females), not suffering from any cardiac or respiratory pathology, and whose age ranged from 25 to 63 years. These subjects have been positioned in front of the radar with different orientations (their chest, their left, their back and right side facing the radar antenna). The employed system has been able to accurately detect BR and HR, regardless of chests patient orientation towards the radar antenna.

c) A custom-designed CW radar system placed under a bed mattresses has been employed in [20] to continuously measure the vital parameters of various patients without restricting their movements. The developed system has been able to detect the activity of each patient (i.e., entering the bed, getting out of it, or moving inside it) and to continuously measure his/her vital signs in different positions.

d) An FMCW radar has been employed for the estimation of the vital signs of a person experiencing different sleep conditions in [77]. In this case, the radar device has been positioned on the ceiling of a room in front of the bed on which a static patient was lying down in different positions or was performing simple activities; this allowed to emulate real-life sleep conditions. The measurements have been acquired on eleven patients (whose age ranged from 25 to 55 years); a good correlation has been found between the radar-based estimates of vital signs and those provided by a reference device. These results, together with those illustrated in [20], have evidenced that radar-based systems can be very useful in various healthcare applications (e.g., in the study of sleep apnoea, in the monitoring of bedridden patients and, more in general, in the monitoring of hospitalized patients).

e) The use of a dual-frequency ${ }^{15} \mathrm{CW}$ microwave radar for vital sign estimation inside an ambulance has been studied in [75]. The measurements have been acquired on eight healthy male subjects whose age ranged from 21 to 24 years. Each of the subjects was lying down on a stretcher contained inside an isolator; therefore, his respiratory and cardiac activities were monitored from outside the isolator. The employed radar system has been able to measure HR and BR of the considered patient with good accuracy, in both static and dynamic conditions of the ambulance. Note that the innovative method proposed for vital sign detection could be very useful in other scenarios (e.g., in the monitoring of infectious patients).

f) Various results about the use of an UWB radar system for monitoring the BR of six neonates ${ }^{16}$ in a neonatal intensive care unit have been illustrated in [86]. In all the experiments, the employed radar system has been covered with a white plastic cap and has been hung at the end of a specially designed arm placed on the top of a tripod; moreover, it has been placed at a distance of $35 \mathrm{~cm}$ from the chest of each subject. Each of the neonates has been placed in a supine position inside an open-air crib, and his/her torso has been covered with a blanket. An high accuracy has been achieved, despite the small movements of the babies under test.

\section{VI.C. Heart sounds monitoring}

Currently, the standard reference tool for continuous cardiac monitoring is electrocardiography; it requires touch-based wiring of patient skin. In clinical practice, a series of pathological processes would certainly benefit from contactless monitoring; these conditions may include patients with an infections or a sepsis (e.g. infected by SARS CoV-2), or patients with mental disorders that do not allow conventional monitoring

\footnotetext{
${ }^{15}$ The considered radar system operated at both $10 \mathrm{GHz}$ and $24 \mathrm{GHz}$.

${ }^{16}$ In this case, the subjects under test were two males and four females with a median gestational age of 38 weeks and a median birth weight of $3100 \mathrm{~g}$.
} 
according to cardiology settings. In cardiac intensive unit or intermediate care unit, continuous monitoring of heartbeat is a common practice, because detection and prevention of critical states of health can lead to timely therapeutic interventions, with the result of a better outcome. In fact, several cardiac conditions could be immediately harmful and potentially fatal (e.g., cardiac arrhythmias, heart attacks, acute heart failure, and stroke), thus requiring prompt detection of cardiac or respiratory anomalies. Recently, in the field of radarbased systems for vital sign monitoring, an effort has been made to take a step forward and, in particular, to detect heart sounds [87-89]. The classification of normal or abnormal heart sounds has been investigated in [87]. In that manuscript, it has been shown that the heart signal recorded by a custom-designed CW radar system is highly correlated with the signal registered by a phonocardiograph, used as reference. Based on this correlation, each period of the recorded radar signal can be divided in the different temporal phases of the cardiac activity, namely into systole and diastole (see Par. III.A.); this procedure is called heart sound segmentation. After recognizing systole and diastole in the received radar signal, a reliable detection of normal or abnormal heart sounds can be accomplished using an Long Short Time Memory (LSTM) network for heart sound segmentation; this has been shown in [88], where an heterogeneous dataset of recorded heart sounds and vital signs acquired through a CW Doppler radar has been used [89]. The measurements of this dataset originate from multiple subjects in different positions of their bodies and in various conditions, such as during breath-holding, during speaking and after post-exercise. The obtained results have evidenced that more than $90 \%$ of the recordings were of high quality, and that the correlation between radar and ECG signals was almost perfect. As far as the impact of body position is concerned, it has been found that, in general, heart sounds can be detected in almost all the considered positions; however, the measurability at a certain position changes from person to person. This is partly due to the fact body anatomy, and in particular, heart position inside chest, may show some variations from person to person.

Further analysis should be performed to achieve solid results, as these represent only preliminary data about the innovative radar-based detection of heart sounds. This technology could lead to a new way of noninvasive cardiac monitoring and could be revolutionary in the cardiology settings. Currently, during medical visits, cardiologists use a stethoscope for heart evaluation; this tool allows them to check for sounds which may indicate pathological changes in the heart or the heart valves. However, the validity of the assessment strongly depends on the experience of the physician. An objective, operator-independent and automated analysis of heart sounds accomplished through radar technology would be very useful; note also that, in this case, the availability of large datasets for big data analysis would be very useful.

\section{Current Trends}

In this section, the most relevant trends in the ongoing research activities on radar systems for vital sign monitoring are described. In particular, we focus on the development of novel digital platforms useful for the implementation of signal processing techniques for radar systems and on the research activities related to the techniques for compensation of random body movements (RBMs).

\section{VII.A. Novel digital platforms usable for digital signal processing in vital sign detection}

The digital platforms usually employed in radar-based estimation of vital signs are central processing units (CPUs), digital signal processors (DSPs), graphic processing units (GPU), application specific integrated circuit (ASIC) based processors, and field programmable gate arrays (FPGAs) [90]. The selection of the device on which specific signal processing techniques are executed determines the speed, cost and power consumption of their implementation; for instance, DSP boards are usually more costly than FPGAs, which, in turn, are cheaper than GPUs. Moreover, in choosing a specific platform, the following technical issues must be carefully taken into account:

a) In medical applications, monitoring and estimating HR and BR continuously and, as much as possible, in real-time, may represent a fundamental requirement. This occurs, for instance, when the monitoring of vital parameters of a single person or multiple people need to be accomplished in an hospital room or in a gym; in all these cases, we need to promptly react whenever the vital parameters of a person change significantly and in an unexpected manner. 
b) The algorithms employed for estimating vital signs are expected to become more and more refined in the next few years; it is very likely that their implementation will require hardware platforms able to efficiently manage an higher computational complexity. The use of well known software languages or tools, such as MATLAB, Python or LabVIEW, does not usually lead to computationally efficient implementations. Readers should also keep in mind that, in such conditions, the use of a single CPU may not allow to achieve high throughput, low latency, low resources utilization, and low power consumption. For this reason, it is likely that, in the near future, techniques for estimating vital parameters will be implemented on a dedicated hardware, such as ASICs or FPGAs. If the processing is performed on an FPGA, hardware description languages, such as Verilog, are employed.

Taking into consideration various platforms or an hybrid combination of two heterogeneous platforms is particularly important when, given an algorithm running differently on distinct platforms, multiple constraints need to be fulfilled. In fact, on the one hand, the reconfigurable nature of an FPGA offers a multifunction implementation and a very high efficiency in managing the available computing resources. On the other hand, ASIC-based processing platforms allow to achieve an higher computing speed than other solutions. Thanks to their cost-efficiency and to the reconfigurability, FPGAs are the platforms preferred by researchers for rapid prototyping [91]. For this reason, in the near future, the use of FPGAs is expected to increase steadily in the area of radar systems.

\section{VII.B. Compensation of random body movements}

The random body movements (RBMs) of any person affect the estimate of vital signs provided by contactless devices. These movements, in fact, modulate the radar waveform both in its amplitude and frequency, so distorting the received echoes. This may significantly affect the quality of the estimates of vital signs generated by radar systems. Consequently, RBMs may represent an important obstacle to the adoption of radar technology in certain scenarios.

Recently, two approaches to motion compensation have been proposed. The first approach is an hardware RBM compensation acting at RF front-end level and, consequently, limiting the risks of saturating radar transceivers in the presence of strong echoes [17]. The second one, instead, consists in accomplishing a digital compensation after demodulation; this can be implemented more easily and lends itself to a more precise control. For this reason, in the remaining part of this paragraph, we focus on the last approach only.

One of the easiest strategies to extract the (weak) vital sign components from the radar measurements and removing the distortion due to RBMs is represented by digital filtering. However, this solution is optimal only when the statistical characteristics of the filter input match prior information on which the design of the filter is based; unluckily, such characteristics are normally unknown. For this reason, a more robust solution has been proposed in [92], where an adaptive noise cancellation (ANC) recursive algorithm is employed, in combination with polynomial fitting, in a CW Doppler radar system. It is important to point out that: a) polynomial fitting is used to reconstruct the signal components due to RBMs and that must be subtracted from the overall signal provided by the radar receiver; b) this strategy allows to achieve RBM compensation when a single radar system is used and does not require additional sensors. The use of a multi-radar system for cancelling RBMs have been proposed in [16]. In this case, two CW Doppler radar systems have been put on opposite sides with respect to the body of a given subject; when his/her body was leaning towards one of the radars, it moved away from the other one [16], so that the distance between each radar and the body changed in an opposite manner. Based on this consideration, it has been shown that RBMs can be cancelled by combining the measurements provided by the two radars. The main drawbacks of a multi-radar approach consists in an increase of system complexity, cost and power consumption and in the need of a larger room for the experimental setup. A different approach to RBM compensation relies on the use of an hybrid system including radar and camera [93]. In this case, the information provided by the camera has been used to compensate for the phase distortion due to body movements. Unluckily, this approach has been shown to work well when body movements are regular and deterministic.

As its can be easily inferred from our previous analysis, RBM compensation should be considered as an open research problem since few solutions are available in the technical literature. 


\section{VII.C. Heart rate variability}

The HR estimated by means of a radar system represents a measure of the overall number of heartbeats observed over a given time interval (e.g., over one minute). However, we should not forget that, within a certain observation interval, the temporal distance between two adjacent heartbeats may not remain constant. This phenomenon, known as HRV, is related to heart-brain interactions and is regulated by the autonomic nervous system [94,95]. More specifically, HRV reflects beat-to-beat changes in RR intervals, which depend on the interrelation between sympathetic and vagal tones. In fact, the sinus node, the principal heart's pacemaker, has its own intrinsic activity; however, several external and internal stimuli altering the autonomic balance influence the final HR [96].

Heart rate changes may originate from a variety of conditions such as mental or physical stress, cardiac or noncardiac diseases, or pharmacological or invasive treatments; the respiration-related fluctuation of HR, known as respiratory sinus arrhythmia, is probably the most commonly investigated component of HRV.

Autonomic nervous system imbalance with increased sympathetic and decreased vagal tone has been proven to be associated with higher risk of cardiac mortality. Therefore, HRV has become an important and recognized tool in identifying patients at risk of cardiovascular death [97], and can be considered as a indicator for both physiological conditions and pathological processes, such as depression, diabetic neuropathy and heart failure. Moreover, it can be exploited to monitor post-surgery and post-infarction patients in order to assess the risk of ventricular tachyarrhythmias leading to sudden cardiac death. Nowadays, different methods can be employed to measure HRV; these include a series of simple bedside reflex tests and more advanced computer-based algorithms for detecting spontaneous RR interval changes. The accomplished analysis is usually based on long-term (usually 24 hours) Holter ECG recordings or short-term (usually few minutes) ECG recordings, and aims at avoiding any influence from external stimuli that could affect autonomic nervous tone [98]. In general, an accurate analysis of HRV may require a long observation interval in a clinical environment or in home-care scenarios. The assessment of this phenomenon is based on the evaluation of various time-domain and frequency-domain features (see [77, Tab. 2, Par. 2.6]). Time domain features aim at quantifying the variability in inter-beat intervals, also called normal to normal (NN) peak intervals ${ }^{17}$ and include the standard deviation of normal to normal peak intervals (SDNN)

$$
\mathrm{SDNN} \triangleq \sqrt{\frac{\sum_{i=1}^{\bar{N}}\left(\Delta t^{(i)}-\Delta \bar{t}\right)^{2}}{\bar{N}-1}}
$$

and the root mean square successive difference of intervals (RMSSD)

$$
\mathrm{RMSSD} \triangleq \sqrt{\frac{\sum_{i=2}^{\bar{N}}\left(\Delta t^{(i)}-\Delta t^{(i-1)}\right)^{2}}{\bar{N}-2}} ;
$$

here, $\bar{N}$ is the total number of beats detected in the heart signal, $\Delta t^{(i)}$ is the duration of the time interval between the $(i+1)$-th detected beat and the previous beat, and

$$
\Delta \bar{t} \triangleq \frac{\sum_{i=0}^{\bar{N}} \Delta t^{(i)}}{\bar{N}+1}
$$

is the average duration of the interval between two consecutive beats. If an estimate of the probability density function (in the form of a probability mass function, pmf) of the NN peak intervals is available, a further meaningful feature is represented by the triangular index (TRI), defined as

$$
\mathrm{TRI}=\frac{\bar{N}}{\mathcal{P}_{N N, \max }},
$$

where $\mathcal{P}_{N N, \text { max }}$ is the maximum of the above mentioned pmf [94].

Frequency domain features are usually evaluated through FFT processing, and refer to the low frequency or to the high frequency band. The former accounts for modulations whose period ranges from $7 \mathrm{~s}$ to $25 \mathrm{~s}$,

\footnotetext{
${ }^{17}$ If an ECG signal is considered, NN intervals are represented by the time intervals between adjacent peaks resulting from sinus node depolarizations. In the case of a CW radar system, instead, these intervals can be defined as the temporal distance between two consecutive maxima of the phase vector $\hat{\psi}$ available at the output of the bandpass filter shown in Fig. 8 .
} 
whereas the latter band refer to shorter periods. The presence of a significant component in the HF band is typically a symptom of stress or anxiety.

Radar-based estimation of HRV may represent an appealing and challenging alternative to ECG for monitoring the physical and mental status of patients. However, as far as we know, this topic is addressed by few manuscripts in the technical literature $[3,12,77,78,99,100]$. More specifically, various results about the use of CW Doppler radars for analysing HRV and drowsiness can be found in [3,99] and [12], respectively. The other manuscripts, instead, involve FMCW radars. In particular, an FMCW radar operating in the K-band has been employed in [77] to monitor HRV in eleven patients of different ages during their sleep. The obtained results evidence that the time and frequency features extracted from the radar signal are correlated with those evaluated on the basis of the measurements acquired from a reference sensor. The effect of the coupling between breathing and heartbeat signals on HRV has been investigated in [78]; in this case, an FMCW radar operating at $122 \mathrm{GHz}$ has been used. Finally, a completely novel approach based on deep learning (and, in particular, on LSTM neural networks) has been proposed in [100] to accurately estimate HRV, analysing data collected by a custom-designed, six-port, CW Doppler radar operating at $24 \mathrm{GHz}$.

\section{VII.D. Vital sign-based authentication}

The capability of radars to accurately estimate the vital signs of a person is attractive not only for monitoring the health status of a patient and detect possible diseases, but also for user identity authentication. Nowadays, many people are used to log-in in their own smartphones by simply looking at their camera or thanks to their fingerprint. These approaches can be classified as what you are methods, since they make use of personal traits (biometrics) that are hard to reproduce or mimic. Within this category, facial recognition represents a less robust user authentication method with respect to other physiological biometrics, such as fingerprints or iris scans [101,102]. Authentication methods exploiting radar-based identification of vital signs, instead, are gaining attention because, unlike other physiological biometric-based approaches (e.g., ECG), they do not require direct contact between the human body and the sensor. Moreover, an identity authentication system based on the recognition of breath or heart traits may be sufficiently robust and reliable, since, as evidenced by various studies, the respiratory personality is unique and is preserved over long periods in adult humans at rest $[103,104]$. This can be related to the fact that the physiological structure (e.g., the strength of the diaphragm and intercostal muscles and volume of the thoracic cavity) and the respiratory motions associated with chest movements have specific characteristics for each person.

The use of a CW Doppler radar device for heart-based and breathing-based user authentication has been investigated in [101] and [105-107], respectively. All the proposed methods make use of ML classification algorithms fed by a set of features extracted from the phase vector $\hat{\boldsymbol{\psi}}(46)$. More specifically, in [105] three different sets of features have been used. The most relevant features of the first set are the BR $\hat{b}_{r}$ (see Par. V.A.), the breathing depth, the average speed of exhale and the average speed of inhale. The other two sets of features, instead, aim at monitoring the ratio of inhale and exhale breathing areas, and the breathing mechanism right after and before the apex (full lung volume). Moreover, it has been shown that a K-NN algorithm, trained over a dataset consisting of measurements lasting $60 \mathrm{~s}$ and acquired over six different subjects, is able to recognize the breathing pattern of different people with a good classification score. Better classification results can be obtained by means of a SVM classifier, as shown in $[106,107]$.

Despite these positive results, respiratory-based identity authentication is far from being mature and requires extensive analysis and investigation. In fact, people need to be authenticated under various mental or physical states. Therefore, potential variations occurring in the normal breathing pattern of a person must be taken into account; ignoring them could reduce the identification accuracy, as observed in [107]. For this reason, an heart-based authentication approach has been proposed in [101]. In this case, the heart signal extracted from the phase vector $\hat{\psi}(46)$ has been segmented in different periods, each encompassing a small number of cardiac cycles; within each period, a set of eight features has been manually extracted. The results obtained through an SVM classifier trained on the data acquired over 78 different subjects have confirmed that an authentication method based on radar-based recognition of cardiac motion may be really feasible. 


\section{Some Considerations on Radar Selection and on its Use in Experimental Campaigns}

In this section, we illustrate some important lessons that we have learnt from our experimental work conducted on healthy volunteers in the laboratories of the Department of Engineering "Enzo Ferrari" and the Cardiology Division, Department of Biomedical, Metabolic and Neural Sciences at the University of Modena and Reggio Emilia. First, we focus on the essential requirements that radar devices employed for vital signs monitoring should meet. Then, we provide some guidelines for developing an experimental setup. Finally, we discuss the difficulties encountered in extracting HR from radar measurements and comment on how to assess estimation accuracy.

\section{VIII.A. Fundamental requirements of radar devices}

Nowadays, a number of compact radar devices, not explicitly developed for medical applications and operating at mmwave, are available on the market at various prices. A radar system employed for vital sign monitoring needs to satisfy various technical requirements, that are influenced by the environment in which measurements are acquired [90]. These requirements concern: a) the maximum distance of the radar from the body of the subject under test; b) its operating frequency; c) its displacement resolution; d) its angular resolution. Different values of the parameters mentioned above may have a substantial impact on the achievable accuracy, as illustrated below.

The maximum distance at which the radar should operate depends on the type of application. If longrange detection is required, an high transmission power and/or highly directive antennas should be employed [108]. If FMCW or SFCW radars are adopted, the maximum measurable distance $R_{\max }$ can be evaluated as

$$
R_{\max }=\frac{c}{2 B} N
$$

where $c$ is the speed of light, $B$ is the bandwidth of the radiated signal and $N$ is the overall number of samples acquired in a chirp interval (frequency sweep) by an FMCW (SFCW) radar. It is also worth mentioning that the availability of an RX antenna array allows to increase the maximum detectable distance. In fact, beamforming techniques can be used to constructively combine the signals made available by distinct RX antennas, so improving the SNR of the resulting signal.

The operating frequency $f_{0}$ of the radar influences the phase resolution in a MIMO configuration (see eq. (59)) and the penetration depth of the radiated EM waves through human tissues [17]. Readers should keep in mind that: a) microwave signals are partially reflected and partially absorbed by the human skin [109]; b) the attenuation of the reflected EM field increases with its frequency; c) the penetration through human skin, instead, decreases with frequency (for instance, the skin penetration depth is $2.7 \mathrm{~mm}$ at $10 \mathrm{GHz}$ and just $0.5 \mathrm{~mm}$ at $60 \mathrm{GHz}[110,111])$. Generally speaking, the wave reflection due to body tissues at higher frequencies is stronger than that at lower frequencies. For these reason, it is reasonable to assume that, if a mmwave radar is used, the BR and HR estimation is based on chest and skin displacement due to the cardio-pulmonary activity.

The displacement resolution can be defined as the minimum measurable displacement over two consecutive frames transmitted by the considered radar device (such frames are separated by a time interval lasting $T_{0}$ s; see Par. IV.B.). Based on (59), the displacement $\Delta R_{k+1, k}$ experienced by a point target between the $k$-th frame and the subsequent frame (i.e., the $(k+1)$-th frame) can be expressed as

$$
\Delta R_{k+1, k}=\frac{\lambda}{4 \pi} \Delta \psi_{k+1, k},
$$

where $\Delta \psi_{k+1, k}$ is the phase variation observed in the electromagnetic echo. If we assume that the maximum chest displacement due to breathing is $\delta_{b, M}$ (see Par. III.B.), the inequality

$$
\frac{\Delta R_{k+1, k}}{T_{0}} \geq \frac{2 \delta_{b, M}}{T_{B R}}
$$

should be satisfied to achieve a sufficient resolution in detecting chest movements. The last inequality can be rewritten as

$$
T_{0} \leq \frac{T_{B R}}{2 \delta_{b, M}} R_{k+1, k}
$$



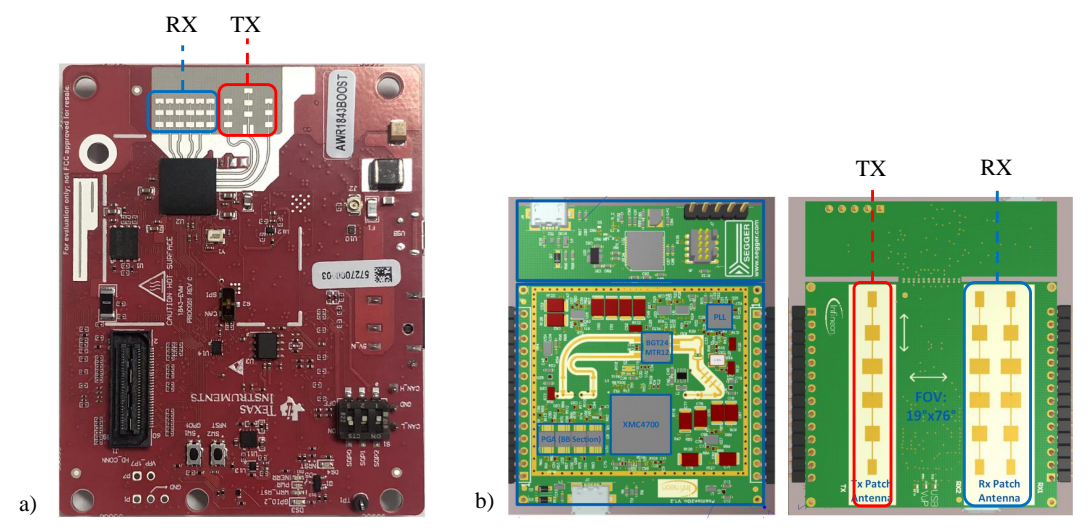

Figure 16: Photo of: a) the IWRxx43 radar front end; b) the Position2Go module.

or, equivalently, as

$$
T_{0} \leq \frac{T_{B R}}{2 \delta_{b, M}} \frac{\lambda}{4 \pi} \Delta \hat{\psi}_{k+1, k}
$$

thanks to (79). If we assume that the maximum displacement of the chest is about $\delta_{b, M} \approx 4 \lambda$ (where $\lambda \cong 4$ $\mathrm{mm}$, if the employed radar system operates at $77 \mathrm{GHz}$ ) and keep in mind that ${ }^{18}$ the inequality $\Delta \psi_{k+1, k}<2 \pi$ must be satisfied to avoid any ambiguity, from (82) it is easily inferred that

$$
T_{0} \leq \frac{T_{B R}}{16},
$$

so that the selected frame interval must be substantially smaller than the breathing period. Similar considerations can be formulated for the displacement due to heart activity. Then, achieving sufficient accuracy in the estimation of $\mathrm{BR}$ and $\mathrm{HR}$ requires the selection of a frame rate $1 / T_{0}$ large enough with respect to the values of these parameters.

Let us focus now on the angular resolution. This parameter plays an important role when a MIMO radar is employed to detect the vital signs of multiple people, characterized by different angular coordinates. The achievable angular resolution is strictly related to the number of virtual channels $N_{V}$ made available by the employed radar system. If this system is endowed with a single ULA characterized by $N_{V}$ equally spaced channels, the angular resolution $\Delta \theta$ can be evaluated as

$$
\Delta \theta=\frac{\lambda}{2 d\left(N_{V}-1\right)} \frac{180^{\circ}}{\pi}
$$

where $d$ is the distance between two adjacent virtual elements; for instance, if $d=\lambda / 4$, the resolution is equal to $2 /\left(N_{V}-1\right)$ rad.

In our measurement campaigns, two colocated FMCW radars, namely the IWRxx43 TI radar [112] and the Position2go (P2G) FMCW radar ${ }^{19}$ [113] have been employed; the former device is manufactured by Texas Instrument, the latter one by Infineon. The front-end of these devices is shown in Fig. 16. It is important to note that in our application $T_{F}=60 \mathrm{~s}$, i.e., the duration of each frame is equal to that of the whole observation interval and the chirp duration $T_{0}=T+T_{R}$ is characterised by a long reset time, since $T_{R}>T$.

The TI radar operates at the frequency $f_{0}=77 \mathrm{GHz}$ and is endowed with an array composed by $N_{T}=3$ $\left(N_{R}=4\right)$ TX (RX) antennas (see Fig. 16-a)); therefore, it makes available a virtual array consisting of $N_{V}=3 \cdot 4=12$ (virtual) antenna elements (see (33)); these elements form two different horizontal ULAs composed by $N_{H U L A_{1}}=8$ and $N_{H U L A_{2}}=4$ virtual channels; therefore, only $N_{V U L A}=2$ aligned channels are available along the vertical direction. The horizontal (i.e., azimuthal) resolution and the vertical (i.e.,

\footnotetext{
${ }^{18}$ This expression is valid if the $\arctan 2(\cdot)$ operator is employed, as illustrated in Par. V.A..

${ }^{19}$ Detailed descriptions of these devices are available at https://www.ti.com/tool/IWR1843B00ST and at https://www. infineon.com/cms/en/product/evaluation-boards/demo-position2go/, respectively.
} 
elevation) resolution are $\Delta \theta \simeq 16.6^{\circ}$ and $\Delta \phi=45^{\circ}$, respectively (see eq. (84)). In our work, the following values of the radar parameters have been selected: a) frequency slope $\mu=86 \mathrm{MHz} / \mu \mathrm{s}$; b) overall number of samples per chirp $N=256$; c) ADC sampling frequency $f_{s}=9 \mathrm{MHz}$; d) chirp duration $T_{0} \approx 60$ msec. This choices entail that: 1) the bandwidth of the radiated signal is $B=\mu T \simeq 2.45 \mathrm{GHz}$, since the ramp up time is $T=N / f_{s}=18.4 \mu \mathrm{s} ; 2$ ) the maximum measurable distance is $R_{\max } \simeq 15.67 \mathrm{~m}$ (see (78)).

The P2G radar, instead, operates at the frequency $f_{0}=24 \mathrm{GHz}$ and is equipped with an array composed by $N_{T}=1$ and $N_{R}=2$ transmit and receive antennas (see Fig. 16-b)). Therefore, a virtual array of $N_{V}=1 \cdot 2=2$ virtual elements (see (33)), forming an horizontal ULA, is available. In our work, the following values of the radar parameters have been selected: a) $\mu=0.78 \mathrm{MHz} / \mathrm{ms}$; b) overall number of samples per chirp $N=256$; c) ADC sampling frequency $f_{s}=1 \mathrm{MHz} ;$ d) chirp duration interval $T_{0} \approx 60$ msec. In this case, the ramp up time is $T=N / f_{s}=256 \mu \mathrm{s}$, the bandwidth of the radiated signal is $B=200$ $\mathrm{MHz}$ and the maximum measurable distance is $R_{\max }=192 \mathrm{~m}$. Therefore, this radar device can be employed for long-range applications, but achieves a lower range resolution than the TI IWR1843 radar.

\section{VIII.B. Data acquisition}

The typical differences between the datasets analysed in the technical literature about radar-based monitoring of vital signs concern (see Table 5): a) the overall number of subjects involved in the data acquisition procedure; b) the position of the subjects with respect to the employed radar (e.g., sitting, lying down, etc.); c) the physical conditions of the involved subjects (e.g., in a rest condition, under strain, sleeping, etc.).

The overall number of subjects ranges from few units to a few dozens. Readers should keep in mind that the analysis of deterministic and ML methods do not usually require a large number of measurements, whereas that of DL techniques needs large and heterogeneous datasets. For instance, the measurements analysed in [114] have been acquired from ten people (more precisely, six males and four females), whose distance from the employed radar system ranged from 20 to $80 \mathrm{~cm}$. On the contrary, a much larger dataset was needed to train and test an LSTM network in [88]. In the last case, the overall acquisition time of the whole dataset is equal to 18900 secs (corresponding to approximately five hours); moreover, the acquired measurements refer to 30 different subjects.

The heterogeneity of the acquired dataset can be improved by observing the considered subjects in different positions (e.g., in front of the radar with the chest facing towards the antennas, or on its left/right side), but always at a fixed distance from the employed radar system, [22]. Subjects can also be placed at different distances from radar systems, but, in this case, their angular coordinates should not change [114, Sect. III].

The measurements analysed in most of the technical literature refer to people breathing at rest. However, especially in last years, contactless systems based on radar technology have been employed for HR and BR monitoring of subjects in different breathing conditions. For instance, the measurements of the dataset employed in [89] have been acquired in apnea, during the Valsalva maneuver ${ }^{20}$ and in two different positions (tilt-up and tilt-down).

The selection of proper reference sensors represents another important technical issue to be considered before starting a measurement campaign. In fact, data collected from reference sensors are always required for the validation of deterministic algorithms [115], [51], or for training learning-based methods [70]. In the measurement campaigns described in the technical literature, various medical instruments, like electrocardiographs and wearable sensors, have been used. Wearable sensors include elastic bands with built-in electrodes [116], micro-electro-mechanical-systems (MEMS), pulse-oximeters or Bragg-gratings sensors [117]. Most of the commercially available wearable sensors are easy to use, since they provide excellent user interfaces and application programming interfaces (APIs) for Python or MATLAB programming environments. However, an important issue to be taken into consideration before selecting a specific reference sensor is the possibility of accurately synchronizing the timing of its measurements with that of the data provided by the employed radar device.

The experimental setup adopted in our measurement campaigns that are currently being accomplished at the Cardiology Division, Department of Biomedical, Metabolic and Neural Sciences at the University of Modena and Reggio Emilia (Hospital of Modena) on healthy volunteers is shown in Fig. 17. Our two radar

\footnotetext{
${ }^{20}$ The Valsava maneuver is performed by moderately forceful attempted exhalation against a closed airway; this can be practically implemented by expiring against a closed glottis.
} 


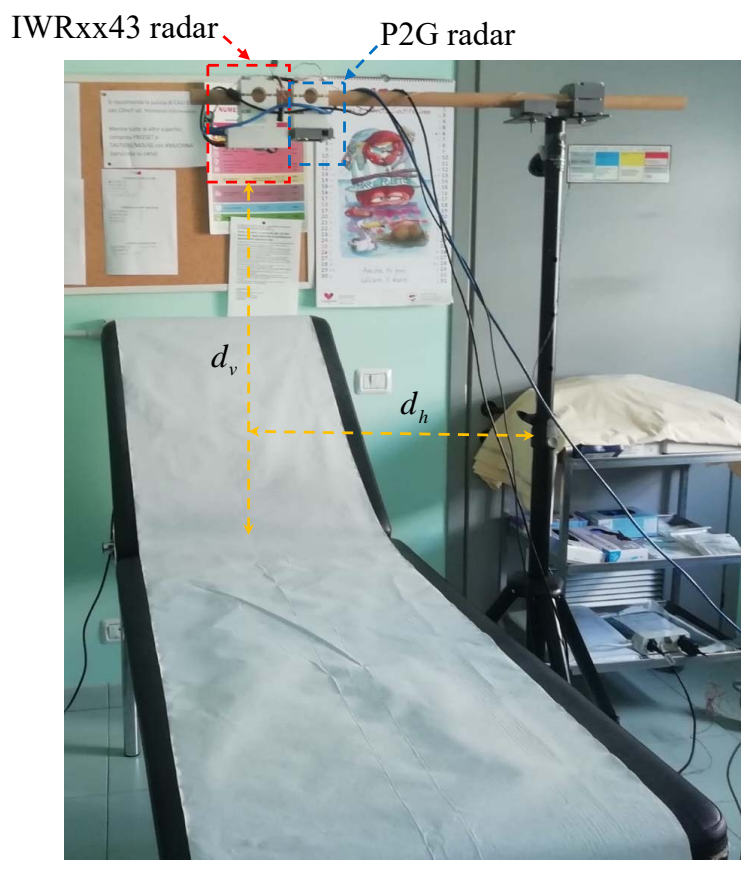

Figure 17: Photo of the setup employed in our measurement campaigns; both the IWRxx43 and the Position2Go radars are used.

sensors are mounted on a wooden bar, which, in turn, is put on a tripod. Moreover, their antenna arrays are oriented towards the chest of each subject, always lying down a bed, whose upper part is slightly tilted. The typical vertical (horizontal) distance $d_{h}\left(d_{v}\right)$ between the radars (the tripod) and the subject under test is approximately equal to $75 \mathrm{~cm}(50 \mathrm{~cm})$; our tripod allows to move up or down the radars, so that the distance between them and the subject can be modified. In our measurements, different positions are being considered for the subject under test. In practice, he/she is sitting or lying down on the bed with different tilting angles; in the first case, his/her movements are very small and this makes the estimation of $\mathrm{HR}$ and BR potentially very accurate, whereas, in the second one, the tension of his/her muscles may affect the estimation of vital sign parameters. In our setup, the reference sensor is the Shimmer3 device manufactured by Shimmer [118]. This device, controlled by means of a simple user interface, is able to send data over a Bluetooth connection and is equipped with five electrodes (right hand side mid-axillary, left hand side mid-axillary, right leg, left leg, V1; see [118, p. 6, Fig. 3-2]), that need to be placed in specific positions of the chest of the subject under test. In our acquisitions, the reference ECG signal is read from the voltage difference between the left leg and right hand side mid-axillary (LL-RA) electrodes. The breathing signal, instead, is generated by measuring the impedance between these two electrodes; in practice, these electrodes are used to inject a weak high frequency $(10 \mathrm{KHz})$ alternating current in the chest tissues and the voltage variations due to chest displacement are measured. Then, an estimate of the chest impedance variations is obtained by computing the ratio between the measured voltage and the injected current; finally, breathing dynamics are inferred from such variations (further details about this method can be found in [119]).

Thanks to the availability of a proper API, Shimmer3 measurements can be easily synchronized in time with those acquired through the IWRxx43 radar or the P2G radar, so that data acquisition can be accomplished in real time. In fact, the Shimmer3 device offers the possibility of sharing the time reference (namely, the CPU timestamp) with our radar devices; this has allowed us to synchronise the reference and the probing signals with great accuracy. As already mentioned above, in our experiment, each data acquisition refers to an observation interval lasting $T_{F}=60 \mathrm{~s}$. However, since the radars and the reference sensor are characterized by different frame rates, the samples of each of the two signals (namely, the ECG and breathing signals) provided by the Shimmer3 sensor are stored in a sequence of vectors, each having size $N_{c, \mathrm{~S}}=15317$, whereas the received signal samples provided by the IWRxx43 (P2G) radar are stored in a 3D matrix of size $N \times N_{V} \times N_{c, \mathrm{TI}}=256 \times 4 \times 997\left(N \times N_{V} \times N_{c, \mathrm{P} 2 \mathrm{G}}=256 \times 2 \times 933\right)$. It is also worth mentioning that, 
in general, if the construction of a dataset requires that acquisitions originating from the given reference sensor and those coming from the employed radar device have the same length, a subsampling or upsampling procedure can be adopted for the reference or the radar signals.

Independently of the nature of the algorithm to be tested on the acquired measurements, it is highly recommended to build up a reasonably large dataset, since the chest dynamics of distinct subjects can exhibit very different characteristics. Moreover, in our measurement campaigns, in order to guarantee a sufficient variability in the generated dataset, the subjects under test are being observed in different conditions. In practice, the following conditions are being considered in our data acquisitions: a) breathing normally, at rest; b) during inspiratory (expiratory) apnoea (for as long as he/she could, ending the acquisition with normal breathing); c) hyperventilating (for as long as he/she could); d) during the Valsalva maneuver; e) after making an effort. In the last case, the subject under test was required to move up a step and down from it for at least one minute).

\section{VIII.C. Heart rate estimation}

Extracting HR from radar measurements represents a substantially more challenging task than BR estimation. In this paragraph we apply the estimation methods described in Par. 1) to the measurements acquired through our two FMCW radar systems described in Par. VIII.A.. Our objective is estimating the BR and HR of a single subject lying down on a bed at a distance $R_{r e f}=d_{v}=0.75 \mathrm{~cm}$ from the radar sensor; in doing so, the experimental setup described in Par. VIII.B. and shown in Fig. 17 is adopted. The signal samples acquired through the four (two) VAs of the IWRxx43 (P2G) radar in the $k$-th chirp interval, with $k=0,1, \ldots, N_{c, \mathrm{x}}-1$ and $\mathrm{x}=\mathrm{TI}(\mathrm{P} 2 \mathrm{G})$ are processed by a beamforming algorithm [37]; this allows to constructively combine the echoes impinging on the RX array along a specific direction and to produce a single $N$-th dimensional column vector $\mathbf{x}_{k}$; this vector feeds the range estimation and bin selection block shown in Fig. 9. The processing accomplished by the last block is based on (60) (a tape-meter is used to compute the reference range $\left.R_{\text {ref }}\right)$. Moreover, this block produces the couple $(\hat{l}[k], \hat{\psi}[k])$, consisting of the bin index $\hat{l}[k]$ and the phase estimate $\hat{\psi}[k]$, for any $k$. It is worth mentioning that: a) that an estimate $\hat{R}[k]$ of the target range is evaluated as

$$
\hat{R}[k]=\hat{l}[k] K_{m},
$$

where

$$
\hat{l}[k]=\arg \max _{l_{m} \leq \bar{l} \leq l_{M}}|X[l, k]|^{2},
$$

$X[l, k]$ is defined by $(60), l_{m}=21, l_{M}=33, K_{m}=32.6\left(l_{m}=2, l_{M}=3, K_{m}=2.6\right)$ for $x=\mathrm{TI}(x=\mathrm{P} 2 \mathrm{G})$; b) $N_{0}=512$ and $R_{m}=0.65 \mathrm{~m}, R_{M}=1 \mathrm{~m}$ have been selected for both radar systems. Note that $\hat{R}[k](85)$ does not necessary coincide with the reference range $R_{r e f}$, since:

a) Some errors are unavoidably introduced in the range measurement procedure. In fact, it not easy to identify exactly the point of the chest on which the beam radiated by the employed radar is focused, since the antennas of both radar devices are not highly directive.

b) The range estimate $\hat{R}\left[k_{1}\right]$ computed in the $k_{1}$-th chirp interval may be slightly different ${ }^{21}$ from the estimate $\hat{R}\left[k_{2}\right]$ obtained in the $k_{2}$-th chirp interval, with $k_{1} \neq k_{2}$.

The elements of the unwrapped phase vector

$$
\hat{\psi} \triangleq\left[\hat{\psi}[0], \hat{\psi}[1], \ldots, \hat{\psi}\left[N_{c, \mathrm{x}}-1\right]\right]^{T},
$$

computed on the basis of the measurements that have been acquired through the TI (P2G) radar in a single observation interval, are represented by the red (green) line in Fig. 18 (note that in order to ease the interpretation of these results, the elements of the two phase vectors have been normalised ${ }^{22}$ ); in this case, AD followed by DC offset removal and phase unwrapping is employed. The results illustrated in Fig.

\footnotetext{
${ }^{21}$ Note that, in principle, information about chest displacement are contained in the sequence $\left\{R[0], R[1], \ldots, R\left[N_{c, \mathrm{x}}-1\right]\right\}$, but, in general, its elements are too noisy for a reliable detection of vital signs.

${ }^{22} \mathrm{~A}$ min-max normalization has been applied to the vector $\hat{\psi}(87)$; the $k$-th element $\bar{\psi}[k]$ of the normalised phase vector is evaluated as $\bar{\psi}[k]=(\hat{\psi}[k]-\min (\hat{\boldsymbol{\psi}})) /(\max (\hat{\boldsymbol{\psi}})-\min (\hat{\boldsymbol{\psi}}))$, with $k=0,1, \ldots, N_{c, \mathrm{x}}-1$.
} 


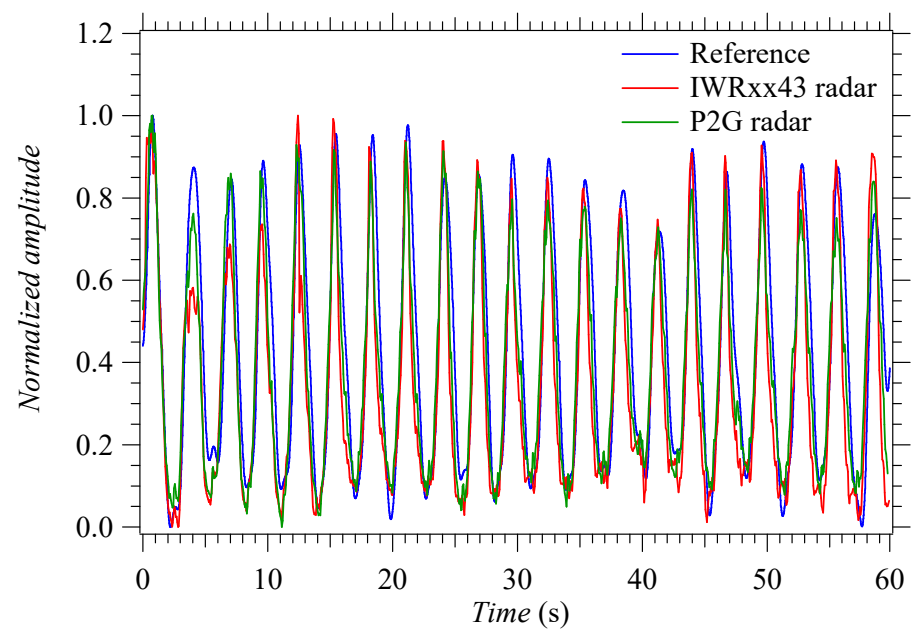

Figure 18: Representation of the breath signal acquired through our reference sensor (blue line), and of the unwrapped phase extracted from the associated IWRxx43 radar measurement (red line) and P2G radar measurement (green line).

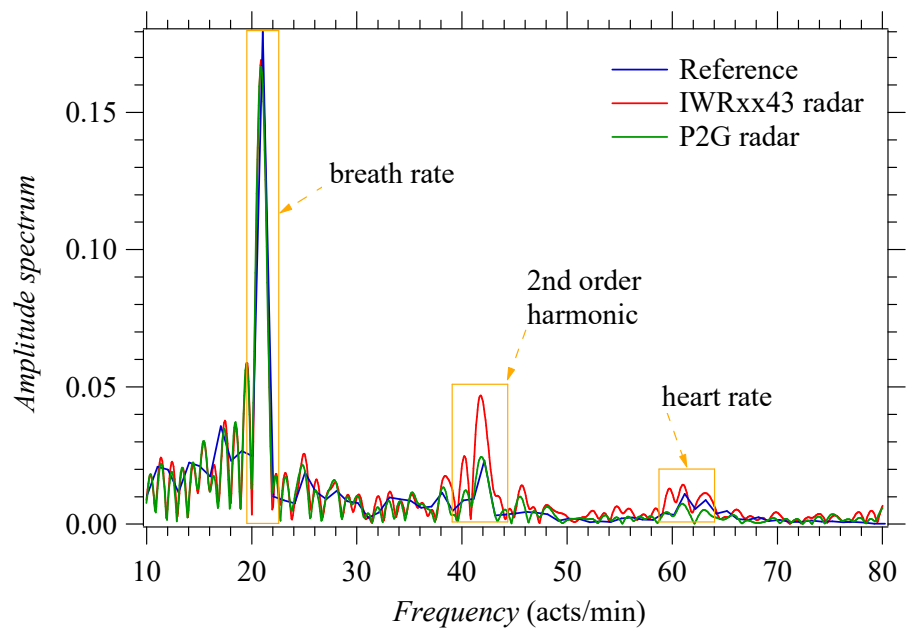

Figure 19: Representation of the amplitude spectrum of the breath signal acquired through our reference sensor (blue line), and of the unwrapped phases extracted from the associated IWRxx43 radar measurement (red line) and P2G radar measurement (green line). 
18 deserve the following comments: a) the two radar signals represent the dynamics of the chest due to both breathing and cardiac activities; b) the phase trajectories computed on the basis of the measurements provided by the two radars overlap, even if these devices operate at different frequencies, and exhibit similar evolutions as the signal provided by our reference sensor (Shimmer3; see the blue line appearing in Fig. 18). The amplitude spectrum generated by applying an FFT of order $N_{0}^{\prime}=N_{c, \mathrm{~S}}=15317$ to each of the signals represented in Fig. 18 is shown in Fig. 19. These results evidence that: a) the spectral peak associated with breathing (in correspondence of $21 \mathrm{acts} / \mathrm{min}$ ) is much larger than the one related to heart beat (visible at approximately 61 acts $/ \mathrm{min}$ ); b) the second order harmonic of breathing is clearly visible at approximately $21 \times 2=42 \mathrm{acts} / \mathrm{min}$, whereas its third harmonic is expected at $21 \times 3=63 \mathrm{acts} / \mathrm{min}$. The heart signal can be extracted from the unwrapped phase through band-pass filtering [21]. In this case, a fourth order band-pass Butterworth is used to select the spectral components whose frequencies belong to the interval $[0.91 \mathrm{~Hz}, 3 \mathrm{~Hz}]$; its output is shown, for both radars, in Fig. 20 (the ECG signal generated by the reference sensor is also represented). Note that, in the considered observation interval, the peak-to-peak period of the radar waveforms is comparable to the NN peak interval characterizing the ECG signal. However, the heart and the ECG signal are not perfectly aligned; this suggests that the heart frequency, i.e. the distance between two consecutive peaks, is not completely stable during the observation time. This is confirmed by the amplitude spectrum of the bandpass filter output generated in response to the two radar signals; the resulting two spectra are represented in Fig. 21, where the amplitude spectrum of the response of the same band-pass filter to the breathing signal acquired by the reference sensor is also shown. Note that, in all these amplitude spectra, three spectral peaks are visible between 60 and 70 acts/min, i.e. in the frequency range in which the spectral contribution due to heartbeat is expected. This problem originates from the fact that: a) the third order harmonic of the breathing signal is close to the fundamental frequency of the heart beat, so that the spectral contribution of the former signal may partially overlap with that of the latter one; b) as suggested by the time-domain signals shown in Fig. 18, the HR changes during our observation interval (lasting $60 \mathrm{~s}$ ) and this entails some spectral broadening. In principle, the last phenomenon can be mitigated by reducing the duration of the observation interval over which spectra are computed; however, an excessive shortening of this interval may lead to an inaccurate estimation of the frequency components due to heart activity.

Finally, it is worth noting that the use of a band-pass filter represents a conceptually simple solution to the problem of extracting the HR components from the phase signal provided by a radar device. Actually, this filter needs to be adapted to the specific conditions of the patient under test. For instance, if his/her BR increases unexpectedly (for instance, in the case of hyperventilation), the spectral components associated with the breathing activity may not be cancelled by this filter and may overlap with the components due to heart beat.

\section{VIII.D. Estimation accuracy}

Let us suppose that an $\bar{N}$-dimensional set $\mathcal{D} \triangleq\left\{\left(\hat{b}_{r}, \hat{h}_{r}, b_{r}, h_{r}\right) ; r=0,1, \ldots, \bar{N}-1\right\}$, where $\hat{b}_{r}$ and $\hat{h}_{r}$ denote the estimates of the BR $b_{r}$ and of $\mathrm{HR} h_{r}$, respectively, is available after that all our radar-based measurements have been processed. Then, the accuracy achieved in vital sign estimation can be assessed by evaluating the mean absolute error (MAE)

$$
\hat{\varepsilon}_{m, x} \triangleq \frac{1}{\bar{N}} \sum_{i=0}^{\bar{N}-1}\left|\hat{x}_{r}[i]-x_{r}[i]\right|,
$$

the peak absolute error (PAE)

$$
\hat{\varepsilon}_{x} \triangleq \arg \max _{0 \leq i \leq \bar{N}-1}\left|\hat{x}_{r}[i]-x_{r}[i]\right|,
$$

and the root mean square error (RMSE)

$$
\bar{\varepsilon}_{x} \triangleq \sqrt{\frac{\sum_{i=0}^{\bar{N}-1}\left(\hat{x}_{r}[i]-x_{r}[i]\right)^{2}}{\bar{N}}}
$$




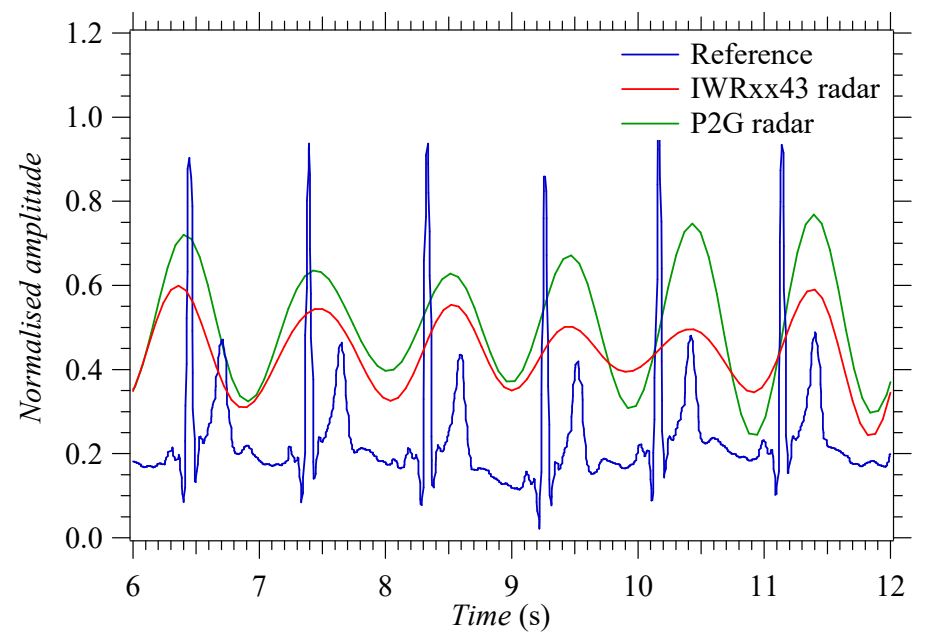

Figure 20: Representation of the ECG signal acquired through our reference sensor (blue line), of the heart signal extracted from the associated IWRxx43 radar measurement (red line) and P2G radar measurement (green line).

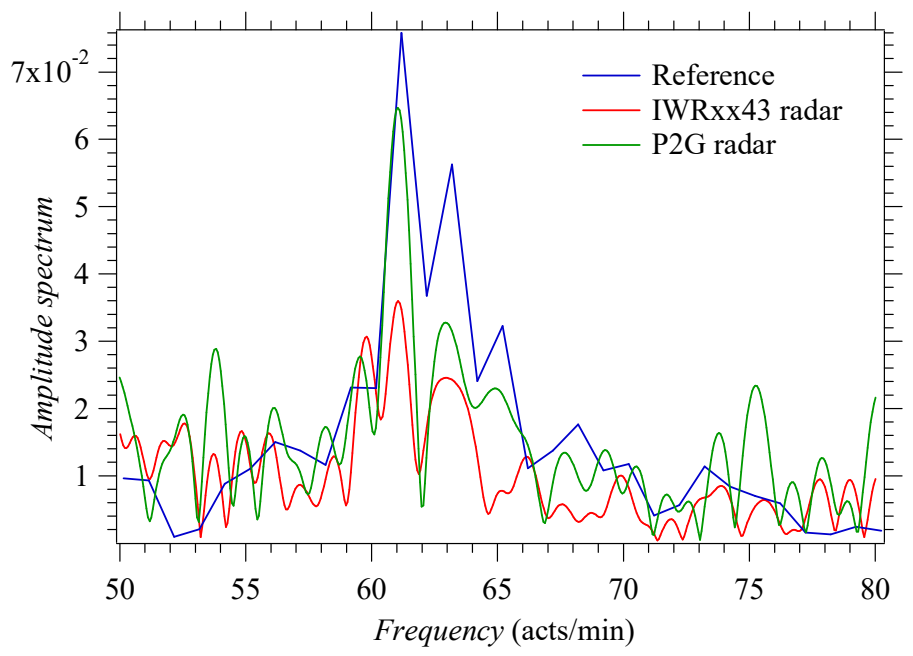

Figure 21: Representation of the amplitude spectrum of the heart signal acquired through our reference sensor (blue line), and of the unwrapped phase extracted from the associated IWRxx43 radar measurement (red line) and P2G radar measurement (green line). 
Table 6: Root mean square error $\left(\bar{\varepsilon}_{x}\right)$, peak absolute error $\left(\hat{\varepsilon}_{x}\right)$, mean absolute error $\left(\hat{\varepsilon}_{m, x}\right)$, correlation of variation $\left(\bar{\gamma}_{x}\right)$ and Pearson coefficient $\left(\rho_{x}\right)$ referring to the BR (HR) estimates $\left\{\hat{b}_{r}\right\}\left(\left\{\hat{h}_{r}\right\}\right)$ computed on the basis of our data set (the measurement unit, m.u., is specified for each parameter).

\begin{tabular}{cccc}
\hline Errors & m.u. & TI Radar & P2G Radar \\
\hline$\left(\bar{\varepsilon}_{b}, \bar{\varepsilon}_{h}\right)$ & acts/ min & $(0.75,1.90)$ & $(1.15,1.70)$ \\
\hline$\left(\hat{\varepsilon}_{b}, \hat{\varepsilon}_{h}\right)$ & acts/min & $(2.80,4.80)$ & $(5.50,4.00)$ \\
\hline$\left(\hat{\varepsilon}_{m, b}, \hat{\varepsilon}_{m, h}\right)$ & acts/min & $(0.45,1.60)$ & $(0.60,1.40)$ \\
\hline$\left(\bar{\gamma}_{b}, \bar{\gamma}_{h}\right)$ & $\%$ & $(4.40,3.24)$ & $(6.80,2.90)$ \\
\hline$\left(\bar{\rho}_{b}, \bar{\rho}_{h}\right)$ & $\%$ & $(90,82)$ & $(84,85)$ \\
\hline
\end{tabular}

where $x=b(x=h)$ if $\mathrm{BR}(\mathrm{HR})$ is considered. Other relevant parameters are represented by the correlation of variation $(\mathrm{CV})$

$$
\bar{\gamma}_{x} \triangleq \frac{\bar{\varepsilon}_{x}}{\frac{1}{N} \sum_{i=0}^{\bar{N}-1} \hat{x}_{r}[i]}
$$

and the Pearson coefficient

$$
\rho_{x} \triangleq \frac{\sum_{i=0}^{\bar{N}-1}\left(\hat{x}_{r}[i]-\bar{x}_{r}\right)}{\sqrt{\sum_{i=0}^{\bar{N}-1}\left(\hat{x}_{r}[i]-\bar{x}_{r}\right)^{2}}} \frac{\sum_{i=0}^{\bar{N}-1}\left(x_{r}[i]-\bar{x}_{r}^{\prime}\right)}{\sqrt{\sum_{i=0}^{\bar{N}-1}\left(x_{r}[i]-\bar{x}_{r}^{\prime}\right)^{2}}},
$$

where

$$
\begin{aligned}
& \bar{x}_{r}=\frac{1}{\bar{N}} \sum_{i=0}^{\bar{N}-1} \hat{x}_{r}[i], \\
& \bar{x}_{r}^{\prime}=\frac{1}{\bar{N}} \sum_{i=0}^{\bar{N}-1} x_{r}[i]
\end{aligned}
$$

$x=b(x=h)$ if $\mathrm{BR}(\mathrm{HR})$ is considered and $x_{r}[i]$ represents the $\mathrm{BR}(\mathrm{HR})$ provided by the adopted reference sensor. Note that value of the parameter $\rho_{x}$ (92) falls in the interval $[-1,1]$; a positive (negative) unitary value is found when the two available datasets (namely, the datasets generated through the employed radar device and reference sensor) exhibit a positive (negative) correlation, whereas a null value means that they are completely uncorrelated.

Typical values of the MAE, the RMSE, the CV and the Pearson coefficient evaluated in radar-based monitoring of vital signs can be found in $[20,51,77]$. In those manuscripts, a reasonable estimation accuracy is achieved if: a) the MAE and RMSE for BR (HR) estimation are in the order of some acts (beats) per minute; b) the $\mathrm{CV}$ is close to $5 \%$ for both $\mathrm{BR}$ and HR estimation; c) the Pearson coefficient is greater than $70 \%$. It is also important to remember that estimation accuracy can be improved through the development of a proper measurement setup; for instance, a small laser device can be employed to verify that the employed radar device is really pointing toward the center of the chest of the patient under test. Another relevant technical issue to be taken into consideration is represented, as already mentioned in the previous paragraph, by the availability of an accurate synchronization between the radar and the reference sensor; unluckily, if the radar and reference devices have independent local clocks, full synchronization cannot be achieved and an accurate evaluation of the parameters defined above is not possible.

Let us analyse now some results obtained on the basis of a limited set of measurements acquired in our experimental campaigns through the IWRxx43 and P2G FMCW radars, and the Shimmer3 reference sensor. We assume that:

a) The employed setup is the one described in Par. VIII.B.. 


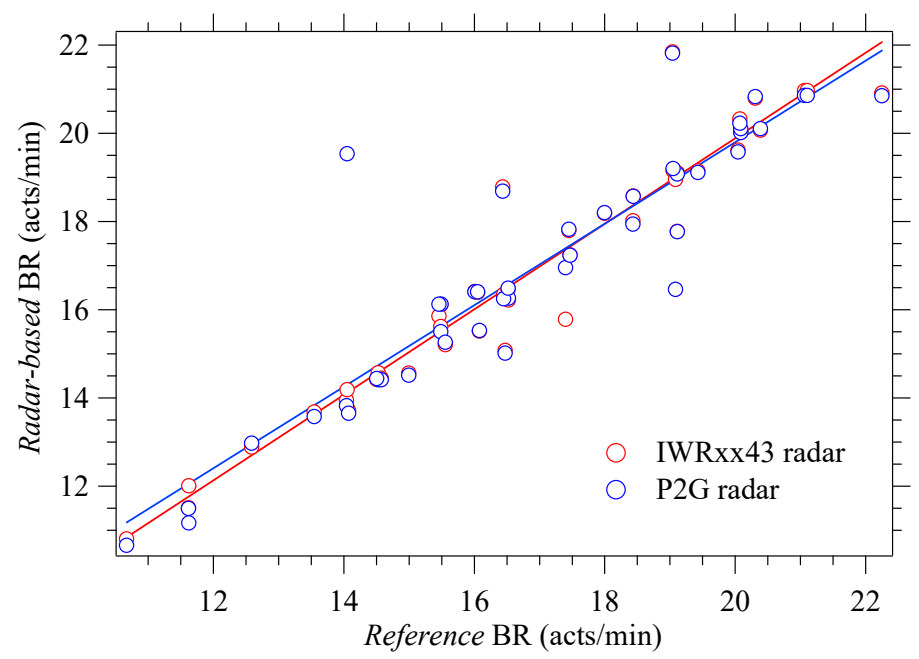

Figure 22: Representation of 45 couples $\left\{\left(b_{r}, \hat{b}_{r}\right)\right\}$; the BRs estimated on the basis of IWRxx43 (P2G) radar are identified by a red (blue) marker.

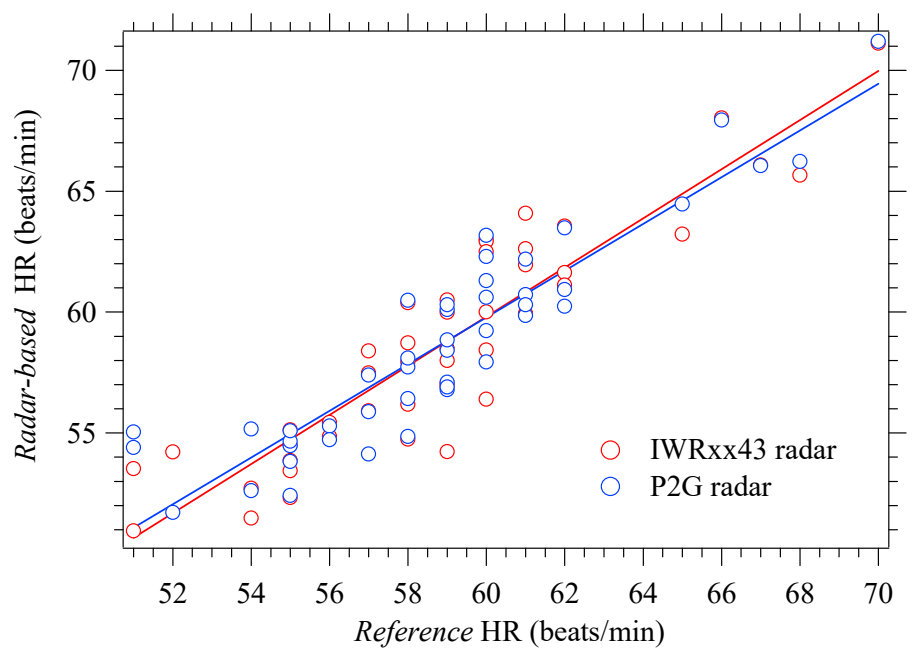

Figure 23: Representation of 45 couples $\left\{\left(h_{r}, \hat{h}_{r}\right)\right\}$; the HRs estimated on the basis of IWRxx43 (P2G) radar are identified by a red (blue) marker. 
b) All our measurements have been acquired from 13 young subjects lying down on a bed and each of them refer to an observation interval lasting $60 \mathrm{~s}$.

c) The HR and BR estimates have been computed by restoring to the processing chain described in Fig. 8 and in Par. 1).

d) The size of the dataset $\mathcal{D}_{\mathrm{TI}}\left(\mathcal{D}_{\mathrm{P} 2 \mathrm{G}}\right)$ referring to the IWRxx43 (P2G) radar is $\bar{N}=45$ (on the average, three distinct measurements have been acquired from each subject).

The values of MAE, PAE, RMSE and CV evaluated on the basis of the available datasets are listed in Table 6, whereas the pairs $\left\{\left(b_{r}, \hat{b}_{r}\right)\right\}\left(\left\{\left(h_{r}, \hat{h}_{r}\right)\right\}\right)$ are represented on a Cartesian plane in Fig. 22 (Fig. 23) for both the IWRxx43 and P2G FMCW radars. Moreover, in Figs. 22 and 23, the lines generated through a linear fitting of the available pairs are also shown. These results deserve the following comments:

a) The estimates of BR and HR are reasonably good;

b) The radar based-estimates are highly correlated with the measurements provided by our reference sensor.

c) The IWRxx43 radar achieves a better accuracy on BR estimation than the P2G radar; on the other hand, the latter device outperforms the former one in HR estimation, since it operate at a lower frequency.

d) The accuracy of the HR estimates is worse than those of BR estimates; note also the RMSEs and peak errors of $\mathrm{HR}$ are in the order of few acts/min.

As far as the last issue is concerned, it is important to keep in mind that: a) the HR frequency may overlap with (or be very close to) the third order harmonic of breath; b) the displacement due to heart is very small and may be not fully detected by our radar devices, if they are not accurately oriented towards the chest of the subject under test.

\section{Conclusions}

Nowadays, a significant body of literature is available in the field of radar-based monitoring of vital signs. This manuscript has offered a broad introduction to this field with the aim of explaining some fundamental concepts, technologies, methods and results to a wide audience. We really hope that our overview of the available radar technologies, of the employed signal processing methods and of the specific applications being considered in medicine will stimulate the interest in radar-based monitoring of vital signs. We believe that radar technology is now mature enough for being taken into account in the medical field. However, there is still ample room for the development of accurate and computationally efficient estimation techniques, and for their implementation on commercial hardware platforms. Readers should also keep in mind that most of the results available in the technical literature refer to a very limited human population and, usually, to healthy subjects. In fact, studies about the monitoring of real patients in realistic medical scenarios are still scarce. Despite this, it has become clear that radar systems can represent a viable alternative to wearable sensors or the only possible option in some critical scenarios, where contactless monitoring is absolutely required. Furthermore, the technological improvements and the advances in processing techniques achieved in recent years have made it possible to overcome various limitations. Therefore, thanks to their capability of continuous and contactless detection, radars may revolutionise patient monitoring in hospitals and in other healthcare facilities in the near future. 


\section{References}

[1] J. Ludikhuize, S. Smorenburg, and Rooij, "Identification of Deteriorating Patients on General Wards; Measurement of Vital Parameters and Potential Effectiveness of the Modified Early Warning Score," J. Crit. Care, vol. 27, pp. 424 7-424 13, 2012.

[2] D. Dias and J. Paulo Silva Cunha, "Wearable Health Devices-Vital Sign Monitoring, Systems and Technologies," Sensors, vol. 18, no. 8, p. 2414, 2018.

[3] S. Suzuki, T. Matsui, S. Gotoh, Y. Mori, B. Takase, and M. Ishihara, "Development of Non-Contact Monitoring System of Heart Rate Variability (HRV) - an Approach of Remote Sensing for Ubiquitous Technology," Proc. of the Int. Conf. on Ergonomics and Health Aspects of Work with Computers, 2009, pp. 195-203.

[4] C. Gu, C. Li, J. Lin, J. Long, J. Huangfu, and L. Ran, "Instrument-Based Noncontact Doppler Radar Vital Sign Detection System Using Heterodyne Digital Quadrature Demodulation Architecture," IEEE Trans. Instrum. Meas., vol. 59, no. 6, pp. 1580-1588, Jun. 2010.

[5] S. M. M. Islam, F. Fioranelli, and V. M. Lubecke, "Can Radar Remote Life Sensing Technology Help Combat COVID-19?" Front. Commun. Netw., vol. 2, p. 3, 2021.

[6] J. C. Lin, "Noninvasive Microwave Measurement of Respiration," Proc. IEEE, vol. 63, no. 10, pp. 1530-1530, Oct. 1975.

[7] T. E. McEwan, "Ultra-Wideband Radar Motion Sensor," Patent 5361070, 1994.

[8] M. Varanini, P. Berardi, F. Conforti, M. Micalizzi, D. Neglia, and A. Macerata, "Cardiac and Respiratory Monitoring through Non-Invasive and Contactless Radar Technique," Proc. of 2008 Computers in Cardiology, 2008, pp. 149-152.

[9] E. Greneker, "Radar Sensing of Heartbeat and Respiration at a Distance with Applications of the Technology," Proc. of Radar 97 (Conf. Publ. No. 449), 1997, pp. 150-154.

[10] E. Staderini, "UWB Radar in Medicine," IEEE Aerosp. Electron. Syst. Mag., vol. 17, pp. 13-18, Feb. 2002.

[11] C. Li, J. Lin, and Y. Xiao, "Robust Overnight Monitoring of Human Vital Signs by a Non-Contact Respiration and Heartbeat Detector," Proc. of the 2006 Int. Conf. of the IEEE Eng. in Medicine and Biology Society (EMBC), Aug. 2006, pp. 2235-2238.

[12] J.-Y. Kim, J.-H. Park, S.-Y. Jang, and J.-R. Yang, "Peak Detection Algorithm for Vital Sign Detection Using Doppler Radar Sensors," Sensors, vol. 19, no. 7, p. 1575, Apr. 2019.

[13] A. Droitcour, V. Lubecke, Jenshan Lin, and O. Boric-Lubecke, "A Microwave Radio for Doppler Radar Sensing of Vital Signs," Proc. of the 2001 IEEE MTT-S Int. Microwave Symp. Digest (Cat. No.01CH37157), vol. 1, May 2001, pp. 175-178.

[14] C. Gu, R. Li, S. B. Jiang, and C. Li, "A Multi-Radar Wireless System for Respiratory Gating and Accurate Tumor Tracking in Lung Cancer Radiotherapy," Proc. of the 2011 Annual Int. Conf. of the IEEE Eng. in Medicine and Biology Society (EMBC), Aug. 2011, pp. 417-420.

[15] C. Li, V. M. Lubecke, O. Boric-Lubecke, and J. Lin, "A Review on Recent Advances in Doppler Radar Sensors for Noncontact Healthcare Monitoring," IEEE Trans Microw. Theory Techn., vol. 61, no. 5, pp. 2046-2060, May 2013.

[16] C. Gu, "Short-Range Noncontact Sensors for Healthcare and Other Emerging Applications: A Review," Sensors, vol. 16, no. 8, p. 1169, Jul. 2016.

[17] M. Kebe, R. Gadhafi, B. Mohammad, M. Sanduleanu, H. Saleh, and M. Al-Qutayri, "Human Vital Signs Detection Methods and Potential Using Radars: A Review," Sensors, vol. 20, no. 5, p. 1454, 2020 . 
[18] E. Cardillo and A. Caddemi, "A Review on Biomedical MIMO Radars for Vital Sign Detection and Human Localization," Electronics, vol. 9, no. 9, 2020.

[19] S. Voinigescu, High-Frequency Integrated Circuits, The Cambridge RF and Microwave Engineering Series. Cambridge: Cambridge University Press, 2013.

[20] S. Schellenberger, K. Shi, F. Michler, F. Lurz, R. Weigel, and A. Koelpin, "Continuous In-Bed Monitoring of Vital Signs Using a Multi Radar Setup for Freely Moving Patients," Sensors, vol. 20, no. 20, p. 5827 , Oct. 2020.

[21] X. Yang, Y. Yu, H. Qian, X. Zhang, and L. Zhang, "Body Orientation and Vital Sign Measurement with IR-UWB Radar Network," Proc. of the 2020 42nd Annual Int. Conf. of the IEEE Eng. in Medicine 86 Biology Society, (EMBC). Montreal, QC, Canada: IEEE, Jul. 2020, pp. 485-488.

[22] G. Sacco, E. Piuzzi, E. Pittella, and S. Pisa, "An FMCW Radar for Localization and Vital Signs Measurement for Different Chest Orientations," Sensors, vol. 20, no. 12, p. 3489, Jun. 2020.

[23] R. O. Bonow, D. L. Mann, D. P. Zipes, and P. Libby, Braunwald's heart disease e-book: A textbook of cardiovascular medicine. Elsevier Health Sciences, 2007.

[24] J. Hall, Guyton and Hall Textbook of Medical Physiology, 13th ed. Philadelphia, PA: Elsevier, 2016.

[25] M. G. Levitzky, "Pulmonary Physiology," Pulmonary Physiology, 7e. New York: McGraw-Hill Medical, 2007.

[26] S. McGee, "Evidence-Based Physical Diagnosis," Evidence-Based Physical Diagnosis, 4th ed., S. McGee, Ed. Philadelphia: Elsevier LTD, Oxford, 2021-08-25, 2021, p. iv.

[27] M. Nosrati and N. Tavassolian, "Accurate Doppler Radar-Based Cardiopulmonary Sensing Using ChestWall Acceleration," IEEE J. Electromagn. RF Microw. Med. Biol., vol. 3, no. 1, pp. 41-47, 2019.

[28] A. De Groote, M. Wantier, G. Cheron, M. Estenne, and M. Paiva, "Chest Wall Motion during Tidal Breathing," J. Appl. Physiol., vol. 83, no. 5, pp. 1531-1537, 1997.

[29] G. Shafiq and K. C. Veluvolu, "Surface Chest Motion Decomposition for Cardiovascular Monitoring," Sci. Rep., vol. 4, no. 1, p. 5093, May 2014.

[30] A. Albanese, L. Cheng, M. Ursino, and N. W. Chbat, "An Integrated Mathematical Model of the Human Cardiopulmonary System: Model Development," Am. J. Physiol.-Heart Circ. Physiol., vol. 310, no. 7, pp. H899-H921, 2016.

[31] D. Wang, S. Yoo, and S. H. Cho, "Experimental Comparison of IR-UWB Radar and FMCW Radar for Vital Signs," Sensors, vol. 20, no. 22, p. 6695, Nov. 2020.

[32] M. Mercuri, Y.-H. Liu, I. Lorato, T. Torfs, A. Bourdoux, and C. Van Hoof, "Frequency-Tracking CW Doppler Radar Solving Small-Angle Approximation and Null Point Issues in Non-Contact Vital Signs Monitoring," IEEE Trans. Biomed. Circuits Syst., vol. 11, no. 3, pp. 671-680, 2017.

[33] M. Skolnik, "Role of Radar in Microwaves," IEEE Trans. Microw. Theory Tech., vol. 50, no. 3, pp. 625-632, 2002.

[34] A. M. Haimovich, R. S. Blum, and L. J. Cimini, "MIMO Radar with Widely Separated Antennas," IEEE Signal Process. Mag., vol. 25, no. 1, pp. 116-129, 2008.

[35] E. Fishler, A. Haimovich, R. Blum, R. Cimini, D. Chizhik, and R. Valenzuela, "Performance of MIMO Radar Systems: Advantages of Angular Diversity," in Conf. Rec. 38th Asilomar Conf. Signals, Sys. Comput., vol. 1, 2004, pp. 305-309.

[36] M. A. Richards, Fundamentals of Radar Signal Processing. New York: McGraw-Hill Education, 2005. 
[37] J. Li and P. Stoica, "MIMO Radar with Colocated Antennas," IEEE Signal Process. Mag., vol. 24, no. 5, pp. 106-114, Sep. 2007.

[38] C. Pfeffer, R. Feger, C. Wagner, and A. Stelzer, "FMCW MIMO Radar System for Frequency-Division Multiple TX-Beamforming," IEEE Trans. Microw. Theory Tech., vol. 61, no. 12, pp. 4262-4274, 2013.

[39] B.-K. Park, O. Boric-Lubecke, and V. M. Lubecke, "Arctangent Demodulation with DC Offset Compensation in Quadrature Doppler Radar Receiver Systems," IEEE Trans. Microw. Theory Tech., vol. 55, no. 5, pp. 1073-1079, 2007.

[40] S. Patole, M. Torlak, D. Wang, and M. Ali, "Automotive Radars: A Review of Signal Processing Techniques," IEEE Sig. Proc. Mag., vol. 34, pp. 22-35, Mar. 2017.

[41] A. Davoli, G. Guerzoni, and G. M. Vitetta, "Machine Learning and Deep Learning Techniques for Colocated MIMO Radars: A Tutorial Overview," IEEE Access, vol. 9, pp. 33 704-33 755, 2021.

[42] P. Bello, "Time-Frequency Duality," IEEE Trans. Inf. Theory, vol. 10, no. 1, pp. 18-33, Jan. 1964.

[43] P. Di Viesti, A. Davoli, G. Guerzoni, and G. M. Vitetta, "Novel Deterministic Detection and Estimation Algorithms for Colocated Multiple-Input Multiple-Output Radars," IEEE Access, vol. 10, pp. 2216$2255,2022$.

[44] K. Naishadham, J. E. Piou, L. Ren, and A. E. Fathy, "Estimation of Cardiopulmonary Parameters from Ultra Wideband Radar Measurements Using the State Space Method," IEEE Trans. Biomed. Circuits Syst., vol. 10, no. 6, pp. 1037-1046, Dec. 2016.

[45] E. Sirignano, A. Davoli, G. M. Vitetta, and F. Viappiani, "A Comparative Analysis of Deterministic Detection and Estimation Techniques for MIMO SFCW Radars," IEEE Access, vol. 7, pp. 129848$129861,2019$.

[46] J. Selva, "ML Estimation and Detection of Multiple Frequencies through Periodogram Estimate Refinement," IEEE Sig. Proc. Lett., vol. 24, no. 3, pp. 249-253, Mar. 2017.

[47] I.-S. Lee, J.-H. Park, and J.-R. Yang, "Detrending Technique for Denoising in CW Radar," Sensors, vol. 21 , no. 19, p. $6376,2021$.

[48] H. Noguchi, H. Kubo, T. Mori, T. Sato, and H. Sanada, "Signal Phase Estimation for Measurement of Respiration Waveform Using a Microwave Doppler Sensor," Proc. of the 2013 35th Annual Int. Conf. of the IEEE Eng. in Medicine and Biology Society (EMBC), 2013, pp. 6740-6743.

[49] J. Wang, X. Wang, L. Chen, J. Huangfu, C. Li, and L. Ran, "Noncontact Distance and AmplitudeIndependent Vibration Measurement Based on an Extended DACM Algorithm," IEEE Trans. Instrum. Meas., vol. 63, no. 1, pp. 145-153, 2014.

[50] Changzhi Li and Jenshan Lin, "Complex Signal Demodulation and Random Body Movement Cancellation Techniques for Non-Contact Vital Sign Detection," 2008 IEEE MTT-S Int. Microwave Symp. Digest. IEEE, 2008, pp. 567-570.

[51] M. Alizadeh, G. Shaker, J. C. M. D. Almeida, P. P. Morita, and S. Safavi-Naeini, "Remote Monitoring of Human Vital Signs Using Mm-Wave FMCW Radar," IEEE Access, vol. 7, pp. 54 958-54968, 2019.

[52] X. Hu and T. Jin, "Short-Range Vital Signs Sensing Based on EEMD and CWT Using IR-UWB Radar," Sensors, vol. 16, no. 12, p. 2025, Nov. 2016.

[53] Y. Xu, S. Dai, S. Wu, J. Chen, and G. Fang, "Vital Sign Detection Method Based on Multiple Higher Order Cumulant for Ultrawideband Radar," IEEE Trans. Geosci. Remote Sens., vol. 50, no. 4, pp. 1254-1265, 2012. 
[54] T. K. Vodai, K. Oleksak, T. Kvelashvili, F. Foroughian, C. Bauder, P. Theilmann, A. Fathy, and O. Kilic, "Enhancement of Remote Vital Sign Monitoring Detection Accuracy Using Multiple-Input Multiple-Output 77 GHz FMCW Radar," IEEE J. Electromagn. RF Microw. Med. Biol., pp. 1-1, 2021.

[55] A. Ahmad, J. C. Roh, D. Wang, and A. Dubey, "Vital Signs Monitoring of Multiple People Using a FMCW Millimeter-Wave Sensor," Proc. of the 2018 IEEE Radar Conf. (RadarConf18), Apr. 2018, pp. 1450-1455.

[56] Y. Wang, Y. Shui, X. Yang, Z. Li, and W. Wang, "Multi-Target Vital Signs Detection Using FrequencyModulated Continuous Wave Radar," EURASIP J. Adv. Signal Process., vol. 2021, no. 1, p. 103, Oct. 2021.

[57] I. Walterscheid, O. Biallawons, and P. Berens, "Contactless Respiration and Heartbeat Monitoring of Multiple People Using a 2-D Imaging Radar," Proc. of the 2019 41st Annual Int. Conf. of the IEEE Eng. in Medicine and Biology Society (EMBC). Berlin, Germany: IEEE, July 2019, pp. 3720-3725.

[58] S. Wang, S. Kueppers, H. Cetinkaya, and R. Herschel, "3D Localization and Vital Sign Detection of Human Subjects with a $120 \mathrm{GHz}$ MIMO Radar," Proc. of the 2019 20th Int. Radar Symp. (IRS), 2019, pp. 1-6.

[59] F. Liang, F. Qi, Q. An, H. Lv, F. Chen, Z. Li, and J. Wang, "Detection of Multiple Stationary Humans Using UWB MIMO Radar," Sensors, vol. 16, no. 11, p. 1922, Nov. 2016.

[60] X. Shang, J. Liu, and J. Li, "Multiple Object Localization and Vital Sign Monitoring Using IR-UWB MIMO Radar," IEEE Trans. Aerosp. Electron. Syst., vol. 56, no. 6, pp. 4437-4450, 2020.

[61] M. Nosrati, S. Shahsavari, S. Lee, H. Wang, and N. Tavassolian, "A Concurrent Dual-Beam PhasedArray Doppler Radar Using MIMO Beamforming Techniques for Short-Range Vital-Signs Monitoring," IEEE Trans. Antennas Propag., vol. 67, no. 4, pp. 2390-2404, 2019.

[62] H. Rohling, "Radar CFAR Thresholding in Clutter and Multiple Target Situations," IEEE Trans. Aerosp. Electron. Syst., vol. AES-19, no. 4, pp. 608-621, 1983.

[63] O. Simeone, A Brief Introduction to Machine Learning for Engineers. Now Foundations and Trends, 2018.

[64] C. M. Bishop, Pattern Recognition and Machine Learning (Information Science and Statistics). Berlin, Heidelberg: Springer-Verlag, 2006.

[65] J. Saluja, J. Casanova, and J. Lin, "A Supervised Machine Learning Algorithm for Heart-Rate Detection Using Doppler Motion-Sensing Radar," IEEE J. Electromagn. RF Microw. Med. Biol, vol. 4, no. 1, pp. $45-51,2020$.

[66] N. T. P. Van, L. Tang, A. Singh, N. D. Minh, S. C. Mukhopadhyay, and S. F. Hasan, "Self-Identification Respiratory Disorder Based on Continuous Wave Radar Sensor System," IEEE Access, vol. 7, pp. $40019-40026,2019$.

[67] N. Malešević, V. Petrović, M. Belić, C. Antfolk, V. Mihajlović, and M. Janković, "Contactless RealTime Heartbeat Detection via $24 \mathrm{GHz}$ Continuous-Wave Doppler Radar Using Artificial Neural Networks," Sensors, vol. 20, no. 8, p. 2351, 2020.

[68] K. Shi, S. Schellenberger, F. Michler, T. Steigleder, A. Malessa, F. Lurz, C. Ostgathe, R. Weigel, and A. Koelpin, "Automatic Signal Quality Index Determination of Radar-Recorded Heart Sound Signals Using Ensemble Classification," IEEE Trans. Biomed. Eng., vol. 67, no. 3, pp. 773-785, Mar. 2020.

[69] C.-Y. Huang, G.-W. Fang, H.-R. Chuang, and C.-L. Yang, "Clutter-Resistant Vital Sign Detection Using Amplitude-Based Demodulation by EEMD-PCA-Correlation Algorithm for FMCW Radar Systems," Proc. of the 2019 49th European Microwave Conference (EuMC). Paris, France: IEEE, Oct. 2019, pp. 928-931. 
[70] S. Wu, T. Sakamoto, K. Oishi, T. Sato, K. Inoue, T. Fukuda, K. Mizutani, and H. Sakai, "PersonSpecific Heart Rate Estimation with Ultra-Wideband Radar Using Convolutional Neural Networks," IEEE Access, vol. 7, pp. 168 484-168 494, 2019.

[71] R. Ichapurapu, S. Jain, M. Kakade, D. Lie, and R. Banister, "A 2.4GHz Non-Contact Biosensor System for Continuous Vital-Signs Monitoring on a Single PCB," Proc. of the 2009 IEEE 8th International Conference on ASIC. IEEE, Nov. 2009, pp. 925-928.

[72] H. Zhao, H. Hong, D. Miao, Y. Li, H. Zhang, Y. Zhang, C. Li, and X. Zhu, "A Noncontact Breathing Disorder Recognition System Using 2.4-GHz Digital-IF Doppler Radar," IEEE J. Biomed. Health Inform., vol. 23, no. 1, pp. 208-217, 2019.

[73] M. Li and J. Lin, "Wavelet-Transform-Based Data-Length-Variation Technique for Fast Heart Rate Detection Using 5.8-GHz CW Doppler Radar," IEEE Trans. Microw. Theory Tech., vol. 66, no. 1, pp. $568-576,2018$.

[74] F. Michler, K. Shi, S. Schellenberger, T. Steigleder, A. Malessa, L. Hameyer, N. Neumann, F. Lurz, C. Ostgathe, R. Weigel, and A. Koelpin, "A Clinically Evaluated Interferometric Continuous-Wave Radar System for the Contactless Measurement of Human Vital Parameters," Sensors, vol. 19, no. 11, p. 2492, May 2019.

[75] S. Suzuki, T. Matsui, H. Kawara, and al, "A Non-Contact Vital Sign Monitoring System for Ambulances Using Dual-Frequency Microwave Radars," Med. Biol. Eng. Comput., vol. 47, no. 1, pp. 101-105, 2009.

[76] L. Anitori, A. de Jong, and F. Nennie, "FMCW Radar for Life-Sign Detection," Proc. of the 2009 IEEE Radar Conf., 2009, pp. 1-6.

[77] E. Turppa, J. M. Kortelainen, O. Antropov, and T. Kiuru, "Vital Sign Monitoring Using FMCW Radar in Various Sleeping Scenarios," Sensors, vol. 20, no. 22, p. 6505, Nov. 2020.

[78] E. Antolinos, F. García-Rial, C. Hernández, D. Montesano, J. I. Godino-Llorente, and J. Grajal, "Cardiopulmonary Activity Monitoring Using Millimeter Wave Radars," Remote Sens., vol. 12, no. 14, 2020.

[79] L. Liu and S. Liu, "Remote Detection of Human Vital Sign with Stepped-Frequency Continuous Wave Radar," IEEE J. Sel. Top. Appl. Earth Obs. Remote Sens., vol. 7, no. 3, pp. 775-782, 2014.

[80] L. Ren, L. Kong, F. Foroughian, H. Wang, P. Theilmann, and A. E. Fathy, "Comparison Study of Noncontact Vital Signs Detection Using a Doppler Stepped-Frequency Continuous-Wave Radar and Camera-Based Imaging Photoplethysmography," IEEE Trans. Microw. Theory Tech., vol. 65, no. 9, pp. 3519-3529, 2017.

[81] W.-C. Su, M.-C. Tang, R. E. Arif, T.-S. Horng, and F.-K. Wang, "Stepped-Frequency ContinuousWave Radar with Self-Injection-Locking Technology for Monitoring Multiple Human Vital Signs," IEEE Trans. Microw. Theory Tech., vol. 67, no. 12, pp. 5396-5405, 2019.

[82] Z. Zhang, Y. Nian, J. Chen, and M. He, "An Experimental Study to Optimize the Stepped-Frequency Continuous-Wave Radar Parameters for Noncontact Multi-Target Vital Sign Monitoring," Proc. of the 2019 IEEE Int. Conf. on Computational Electromagnetics (ICCEM), 2019, pp. 1-4.

[83] F. Khan and S. Cho, "A Detailed Algorithm for Vital Sign Monitoring of a Stationary/Non-Stationary Human through IR-UWB Radar," Sensors, vol. 17, no. 2, p. 290, Feb. 2017.

[84] H. Shen, C. Xu, Y. Yang, L. Sun, Z. Cai, L. Bai, E. Clancy, and X. Huang, "Respiration and Heartbeat Rates Measurement Based on Autocorrelation Using IR-UWB Radar," IEEE Trans. Circuits Syst. II Express Briefs, vol. 65, no. 10, pp. 1470-1474, 2018.

[85] J.-Y. Park, Y. Lee, Y.-W. Choi, R. Heo, H.-K. Park, S.-H. Cho, S. H. Cho, and Y.-H. Lim, "Preclinical Evaluation of a Noncontact Simultaneous Monitoring Method for Respiration and Carotid Pulsation Using Impulse-Radio Ultra-Wideband Radar," Sci. Rep., vol. 9, no. 1, p. 11892, Dec. 2019. 
[86] J. D. Kim, W. H. Lee, Y. Lee, H. J. Lee, T. Cha, S. H. Kim, K.-M. Song, Y.-H. Lim, S. H. Cho, S. H. Cho, and H.-K. Park, "Non-Contact Respiration Monitoring Using Impulse Radio Ultrawideband Radar in Neonates," R. Soc. Open Sci., vol. 6, no. 6, p. 190149, Jun. 2019.

[87] C. Will, K. Shi, S. Schellenberger, T. Steigleder, F. Michler, J. Fuchs, R. Weigel, C. Ostgathe, and A. Koelpin, "Radar-Based Heart Sound Detection," Sci. Rep., vol. 8, no. 1, p. 11551, Dec. 2018.

[88] K. Shi, R. Weigel, A. Koelpin, S. Schellenberger, L. Weber, J. P. Wiedemann, F. Michler, T. Steigleder, A. Malessa, F. Lurz, and C. Ostgathe, "Segmentation of Radar-Recorded Heart Sound Signals Using Bidirectional LSTM Networks," Proc. of the 2019 41st Annual Int. Conf. of the IEEE Eng. in Medicine and Biology Society (EMBC). Berlin, Germany: IEEE, Jul. 2019, pp. 6677-6680.

[89] K. Shi, S. Schellenberger, C. Will, T. Steigleder, F. Michler, J. Fuchs, R. Weigel, C. Ostgathe, and A. Koelpin, "A Dataset of Radar-Recorded Heart Sounds and Vital Signs Including Synchronised Reference Sensor Signals," Sci. Data, vol. 7, no. 1, p. 50, Dec. 2020.

[90] A. Bin Obadi, P. J. Soh, O. Aldayel, M. H. Al-Doori, M. Mercuri, and D. Schreurs, "A Survey on Vital Signs Detection Using Radar Techniques and Processing with FPGA Implementation," IEEE Circuits Syst. Mag., vol. 21, no. 1, pp. 41-74, 2021.

[91] A. Jarrah and M. M. Jamali, "Reconfigurable FPGA/GPU-Based Architecture of Block Compressive Sampling Matching Pursuit Algorithm," J. Circuits Syst. Comput., vol. 24, no. 04, p. 1550055, 2015.

[92] Z.-K. Yang, H. Shi, S. Zhao, and X.-D. Huang, "Vital Sign Detection during Large-Scale and Fast Body Movements Based on an Adaptive Noise Cancellation Algorithm Using a Single Doppler Radar Sensor," Sensors, vol. 20, no. 15, p. 4183, Jul. 2020.

[93] C. Gu, G. Wang, Y. Li, T. Inoue, and C. Li, "A Hybrid Radar-Camera Sensing System with Phase Compensation for Random Body Movement Cancellation in Doppler Vital Sign Detection," IEEE Trans. Microw. Theory Tech., vol. 61, no. 12, pp. 4678-4688, 2013.

[94] Task Force of the European Society of Cardiology the North A Electrophysiology, "Heart Rate Variability," Circulation, vol. 93, no. 5, pp. 1043-1065, 1996.

[95] F. Shaffer and J. P. Ginsberg, "An Overview of Heart Rate Variability Metrics and Norms," Front. Public Health, vol. 5, p. 258, 2017.

[96] K. C. Bilchick and R. D. Berger, "Heart Rate Variability," Journal of Cardiovascular Electrophysiology, vol. 17, no. 6, pp. 691-694, 2006.

[97] M. K. Lahiri, P. J. Kannankeril, and J. J. Goldberger, "Assessment of Autonomic Function in Cardiovascular Disease: Physiological Basis and Prognostic Implications," Journal of the American College of Cardiology, vol. 51, no. 18, pp. 1725-1733, 2008.

[98] I. Cygankiewicz and W. Zareba, "Chapter 31 - heart rate variability," Autonomic Nervous System, ser. Handbook of Clinical Neurology, R. M. Buijs and D. F. Swaab, Eds. Elsevier, 2013, vol. 117, pp. 379-393.

[99] O. Boric-Lubecke, W. Massagram, V. M. Lubecke, A. Host-Madsen, and B. Jokanovic, "Heart Rate Variability Assessment Using Doppler Radar with Linear Demodulation," Proc. of the 2008 38th European Microwave Conf., 2008, pp. 420-423.

[100] K. Shi, T. Steigleder, S. Schellenberger, F. Michler, A. Malessa, F. Lurz, N. Rohleder, C. Ostgathe, R. Weigel, and A. Koelpin, "Contactless Analysis of Heart Rate Variability during Cold Pressor Test Using Radar Interferometry and Bidirectional LSTM Networks," Sci. Rep., vol. 11, no. 1, p. 3025, Feb. 2021.

[101] J. Liu, Y. Chen, Y. Dong, Y. Wang, T. Zhao, and Y.-D. Yao, "Continuous User Verification via Respiratory Biometrics," in IEEE INFOCOM 2020, 2020, pp. 1-10. 
[102] J. Chauhan, Y. Hu, S. Seneviratne, A. Misra, A. Seneviratne, and Y. Lee, "BreathPrint: Breathing Acoustics-Based User Authentication," Proc. of the 15th Annual Int. Conf. on Mobile Systems, Applications, and Services (MobiSys'17). New York, NY, USA: Association for Computing Machinery, 2017, pp. 278-291.

[103] H. J. Proctor DF, "Studies of Respiratory Air Flow; Significance of the Normal Pneumotachogram," Bull Johns Hopkins Hosp., vol. 85, no. 4, pp. 253-280, 1949.

[104] G. Benchetrit, S. Shea, T. Dinh, S. Bodocco, P. Baconnier, and A. Guz, "Individuality of Breathing Patterns in Adults Assessed over Time," Respir. Physiol., vol. 75, no. 2, pp. 199-209, 1989.

[105] A. Rahman, V. M. Lubecke, O. Boric-Lubecke, J. H. Prins, and T. Sakamoto, "Doppler Radar Techniques for Accurate Respiration Characterization and Subject Identification," IEEE J. Emerg. Se.l Top. Circuits Syst., vol. 8, no. 2, pp. 350-359, Jun. 2018.

[106] S. M. M. Islam, A. Rahman, N. Prasad, O. Boric-Lubecke, and V. M. Lubecke, "Identity Authentication System Using a Support Vector Machine (SVM) on Radar Respiration Measurements," Pro. of the 2019 93rd ARFTG Microwave Measurement Conf. (ARFTG), 2019, pp. 1-5.

[107] S. M. M. Islam, A. Sylvester, G. Orpilla, and V. M. Lubecke, "Respiratory Feature Extraction for Radar-Based Continuous Identity Authentication," Proc. of the 2020 IEEE Radio and Wireless Symposium (RWS), 2020, pp. 119-122.

[108] O. Biallawons and J. Klare, "Person Localization by Detection of Breathing with the MIMO Radar MIRA-CLE Ka," Proc. of the 10th European Conference on Synthetic Aperture Radar (EUSAR 2014), 2014, pp. 1-4.

[109] S. Gabriel, R. W. Lau, and C. Gabriel, "The Dielectric Properties of Biological Tissues: III. Parametric Models for the Dielectric Spectrum of Tissues," Phys. Med. Biol., vol. 41, no. 11, pp. 2271-2293, Nov. 1996.

[110] M. G. Amin, "Radar for Indoor Monitoring: Detection, Classification, and Assessment," M. G. Amin, Ed. CRC Press, 2017.

[111] M. Zhadobov, N. Chahat, R. Sauleau, C. Le Quement, and Y. Le Drean, "Millimeter-Wave Interactions with the Human Body: State of Knowledge and Recent Advances," Int. J. Microw. Wirel. Technol., vol. 3, no. 2, pp. 237-247, 2011.

[112] "Texas Instrument Inc." [Online]. Available: https://www.ti.com/

[113] "Infineon GmbH." [Online]. Available: https://www.infineon.com/

[114] Q. Liang, L. Xu, N. Bao, L. Qi, J. Shi, Y. Yang, and Y. Yao, "Research on Non-Contact Monitoring System for Human Physiological Signal and Body Movement," Biosensors, vol. 9, no. 2, 2019.

[115] Y. Ding, X. Yu, C. Lei, Y. Sun, X. Xu, and J. Zhang, "A Novel Real-Time Human Heart Rate Estimation Method for Noncontact Vital Sign Radar Detection," IEEE Access, vol. 8, pp. 88689$88699,2020$.

[116] "Hexoskin Device." [Online]. Available: https://www.hexoskin.com/

[117] M. Fajkus, "Alternative Fiber Optic Sensor Based on Bragg Grating for Heart Rate Monitoring," Int. J. Biosens. Bioelectron., vol. 4, Oct. 2018.

[118] "Shimmer Sensing." [Online]. Available: http://shimmersensing.com/

[119] A. K. Gupta, "Respiration Rate Measurement Based on Impedance Pneumography," 2011.

[120] J. P. A. Ioannidis, K. W. Boyack, and J. Baas, "Updated science-wide author databases of standardized citation indicators," PLOS Biology, vol. 18, no. 10, pp. 1-3, 102020. 